\title{
Automatic transversality and orbifolds of punctured holomorphic curves in dimension four
}

\author{
Chris Wendl*
}

\begin{abstract}
We derive a numerical criterion for $J$-holomorphic curves in 4-dimensional symplectic cobordisms to achieve transversality without any genericity assumption. This generalizes results of Hofer-Lizan-Sikorav [HLS97] and Ivashkovich-Shevchishin [IS99] to allow punctured curves with boundary that generally need not be somewhere injective or immersed. As an application, we combine this with the intersection theory of punctured holomorphic curves to prove that certain geometrically natural moduli spaces are globally smooth orbifolds, consisting generically of embedded curves, plus unbranched multiple covers that form isolated orbifold singularities.
\end{abstract}

Mathematics Subject Classification (2000). Primary 32Q65; Secondary 57R17.

Keywords. Pseudoholomorphic curves, transversality, symplectic cobordisms, symplectic 4-manifolds, contact 3-manifolds.

\section{Contents}

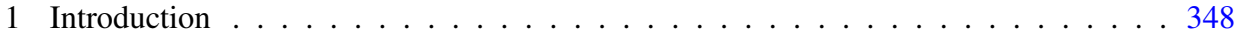

1.1 The setting . . . . . . . . . . . . . . . . . . . . . . . . . . . . . 349

1.2 Local and global transversality results . . . . . . . . . . . . . . 354

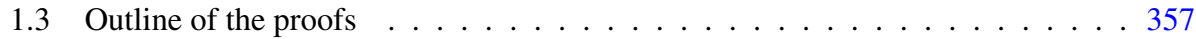

2 Cauchy-Riemann type operators on bundles . . . . . . . . . . . . . 358

2.1 Generalities . . . . . . . . . . . . . . . . . . 358

2.2 The line bundle case . . . . . . . . . . . . . . . . . . . . . 361

3 The normal operator for a holomorphic curve . . . . . . . . . . . . . . 364

3.1 Teichmüller slices and Cauchy-Riemann operators . . . . . . . . . . . . 365

3.2 Functional analytic setup . . . . . . . . . . . . . . . . . . . 369

3.3 The generalized normal bundle . . . . . . . . . . . . . . 378

3.4 Splitting the linearization . . . . . . . . . . . . . . . . . . 380

3.5 The transversality criterion in dimension four . . . . . . . . . . . 385

4 Application to spaces of embedded curves . . . . . . . . . . . . . . . 386

4.1 Intersection theory for punctured holomorphic curves . . . . . . . . . . 387

\footnotetext{
${ }^{*}$ Research partially supported by an NSF Postdoctoral Fellowship (DMS-0603500) and a DFG grant (CI 45/2-1).
} 
4.2 Some covering relations . . . . . . . . . . . . . . . . . . . . . . 391

4.3 Multiply covered limits are immersed . . . . . . . . . . . . . . . . . . . . . . . . . . . 495

Appendix. Counting boundary zeros . . . . . . . . . . . . . . . . . . . . . . . 404

References . . . . . . . . . . . . . . . . . . . . . 406

\section{Introduction}

Applications of pseudoholomorphic curves in symplectic 4-manifolds and contact 3-manifolds often depend on the rather special transversality properties that exist in this low-dimensional setting. Unlike the general situation, where the moduli space is smooth only at somewhere injective curves and only for generic data, certain moduli spaces in dimension 4 are smooth for all data as long as the right numerical criteria are satisfied. For example, suppose $(W, J)$ is any almost complex 4-manifold, $(\Sigma, j)$ is a closed Riemann surface of genus $g$ and $u:(\Sigma, j) \rightarrow(W, J)$ is a pseudoholomorphic curve. The following result was first mentioned by Gromov [Gro85], and later given a complete proof by Hofer-Lizan-Sikorav:

Theorem ([HLS97]). If $u$ is embedded and $c_{1}\left(u^{*} T W, J\right)>0$, then the moduli space of unparametrized pseudoholomorphic curves near $u$ is a smooth manifold of dimension $2 c_{1}\left(u^{*} T W, J\right)+2 g-2$.

Observe that the assumptions in the theorem do not require any data to be generic: rather, the criterion $c_{1}\left(u^{*} T W\right)>0$ implies regularity for uniquely 4-dimensional reasons that are loosely related to positivity of intersections. The dimension of the moduli space is then equal to its so-called virtual dimension, also called the index of $u$, defined as ind $(u)=2 c_{1}\left(u^{*} T W\right)+2 g-2$. Thus $c_{1}\left(u^{*} T W\right)>0$ is equivalent to the condition ind $(u)>2 g-2$, which leads one to summarize results of this type with the motto, "the moduli space is smooth if the index is sufficiently large." Exactly how large the index needs to be depends on the genus: this is the reason why almost all applications of such results (including the one in this paper) principally involve curves of genus zero.

Versions of the theorem above for compact immersed holomorphic curves with boundary were proved in [HLS97], and similar results for immersed punctured curves in symplectizations of contact 3-manifolds also appeared in [HWZ99], [Wen05]. The reason for dealing with immersed curves in particular was that one could then describe a neighborhood of $u$ in the moduli space using sections of its normal bundle and thus reduce the linearization to the so-called normal Cauchy-Riemann operator. The key fact about this operator is that its domain is a space of sections on a complex line bundle, thus the zeroes of these sections can be counted and related to the same topological invariants that appear in the index formula, giving rise to constraints on the kernel and cokernel. A generalization for closed holomorphic curves with critical 
points was carried out in [IS99], where the normal bundle was replaced by a normal sheaf.

In this paper, we establish a transversality criterion that generalizes all of the above results, applying to arbitrary $J$-holomorphic curves with totally real boundary and cylindrical ends in 4-dimensional symplectic cobordisms.

One of the advantages of this approach to transversality is that it applies to more than just somewhere injective curves: in $\S 4$, we will describe a setting in which our criterion, combined with some nontrivial intersection theory, implies that certain moduli spaces are smooth orbifolds, which consist mostly of embedded holomorphic curves but also have isolated singularities consisting of unbranched multiple covers over embedded curves. These moduli spaces arise quite naturally in a geometric setting: they are the building blocks of $J$-holomorphic foliations, cf. [HWZ03], [Wen08].

1.1. The setting. Let $n \geq 2$. In all of what follows, $(W, J)$ will denote a $2 n$ dimensional almost complex manifold with noncompact cylindrical ends, which approach $(2 n-1)$-manifolds $M_{ \pm}$equipped with stable Hamiltonian structures. We now recall the precise definitions.

We use the term stable Hamiltonian structure to mean the collection of data that were introduced in $[\mathrm{BEH}+03]$ as the appropriate setting for pseudoholomorphic curves in cylindrical manifolds. Namely, such a structure $\mathscr{H}=(\xi, X, \omega, J)$ on a $(2 n-1)$-manifold $M$ consists of the following data: ${ }^{1}$

- $\xi$ is a smooth cooriented hyperplane distribution on $M$

- $\omega$ is a smooth closed 2-form on $M$ which restricts to a symplectic structure on the vector bundle $\xi \rightarrow M$

- $X$ is a smooth vector field which is transverse to $\xi$, satisfies $\omega(X, \cdot) \equiv 0$, and whose flow preserves $\xi$

- $J$ is a smooth complex structure on the bundle $\xi \rightarrow M$, compatible with $\omega$ in the sense that $\omega(\cdot, J \cdot)$ defines a bundle metric

Note that, as a consequence of these definitions, the flow $\varphi_{X}^{t}: M \rightarrow M$ of $X$ also preserves the symplectic structure $\left.\omega\right|_{\xi}$, and the special 1-form $\lambda$ associated to $\xi$ and $X$ by the conditions

$$
\lambda(X) \equiv 1, \quad \operatorname{ker} \lambda \equiv \xi,
$$

satisfies $d \lambda(X, \cdot) \equiv 0$. The symplectization $\mathbb{R} \times M$ now admits a natural $\mathbb{R}$-invariant almost complex structure $\tilde{J}$, defined by the conditions

$$
\tilde{J} \partial_{a}=X,\left.\quad \tilde{J}\right|_{\xi}=J
$$

\footnotetext{
${ }^{1}$ The inclusion of $J$ in the data is somewhat nonstandard but convenient for our purposes. The data $(\xi, X, \omega)$ are equivalent to the definition of a framed Hamiltonian structure stated in [EKP06], with the exception that the latter requires $\omega$ to be exact; here it need only be closed.
} 
where $a$ denotes the coordinate on the $\mathbb{R}$-factor and $\partial_{a} \in T(\mathbb{R} \times M)$ is the corresponding unit vector field.

Recall that a $T$-periodic orbit $x: \mathbb{R} \rightarrow M$ is nondegenerate if the linearized return $\left.\operatorname{map} d \varphi_{X}^{T}(x(0))\right|_{\xi_{x(0)}}$ does not have 1 as an eigenvalue. More generally, a Morse-Bott manifold of T-periodic orbits is a submanifold $N \subset M$ tangent to $X$ such that $\left.\varphi_{X}^{T}\right|_{N}$ is the identity, and for all $p \in N$,

$$
T_{p} N=\operatorname{ker}\left(d \varphi_{X}^{T}(p)-\mathbb{1}\right) .
$$

We will say that an orbit with period $T$ is Morse-Bott if it is contained in a MorseBott manifold of $T$-periodic orbits; note that this manifold could be a circle, meaning the orbit is nondegenerate. Moreover, $X$ itself is said to be Morse-Bott (or nondegenerate) if every periodic orbit of $X$ is Morse-Bott (or nondegenerate).

We now fix two closed ( $2 n-1)$-manifolds $M_{ \pm}$with stable Hamiltonian structures $\mathscr{H}_{ \pm}=\left(\xi_{ \pm}, X_{ \pm}, \omega_{ \pm}, J_{ \pm}\right)$and associated data $\lambda_{ \pm}$and $\tilde{J}_{ \pm}$, as well as an almost complex $2 n$-manifold $(W, J)$ which decomposes

$$
W=E_{-} \cup_{M_{-}} W_{0} \cup_{M_{+}} E_{+}
$$

so that

- $W_{0}$ is a compact $2 n$-manifold with boundary $\partial W_{0}=M_{-} \sqcup M_{+}$

- $\left(E_{-}, J\right) \cong\left((-\infty, 0] \times M_{-}, \tilde{J}_{-}\right)$and $\left(E_{+}, J\right) \cong\left([0, \infty) \times M_{+}, \tilde{J}_{+}\right)$

Fix also a totally real submanifold $L \subset W$.

Near $\partial E_{ \pm} \subset E_{ \pm}$, the data $\mathscr{H}_{ \pm}$define natural symplectic forms $\omega_{ \pm}+d\left(a \lambda_{ \pm}\right)$ which can be extended (non-uniquely) over $E_{ \pm}$. Then given any symplectic form $\omega$ on $W_{0}$ that attaches smoothly to $\omega_{ \pm}+d\left(a \lambda_{ \pm}\right)$at $\partial W_{0}$, we denote by $\mathcal{J}_{\omega}\left(W, \mathscr{H}_{+}, \mathscr{H}_{-}\right)$ the space of almost complex structures $J$ on $W$ that are compatible with $\omega$ on $W_{0}$ and satisfy the conditions above. ${ }^{2}$

We will consider pseudoholomorphic (or J-holomorphic) curves

$$
u:(\dot{\Sigma}, j) \rightarrow(W, J),
$$

where $\dot{\Sigma}=\Sigma \backslash \Gamma,(\Sigma, j)$ is a compact connected Riemann surface with boundary, $\Gamma \subset$ int $\Sigma$ is a finite set of interior punctures, ${ }^{3}$ and by definition $u$ satisfies the nonlinear Cauchy-Riemann equation $T u \circ j=J \circ T u$ and boundary condition $u(\partial \Sigma) \subset L$. We also will assume $u$ is asymptotically cylindrical, which means the following. Partition the punctures into positive and negative subsets

$$
\Gamma=\Gamma^{+} \cup \Gamma^{-},
$$

\footnotetext{
${ }^{2}$ The symplectic form $\omega$ will play almost no role in anything that follows, but becomes important in applications, e.g. it yields compactness results as in [BEH+03].

${ }^{3}$ For brevity we're leaving out the case of punctures on the boundary, though this can presumably be handled by similar methods.
} 
and at each $z \in \Gamma^{ \pm}$, choose a biholomorphic identification of a punctured neighborhood of $z$ with the half-cylinder $Z_{ \pm}$, where

$$
Z_{+}=[0, \infty) \times S^{1} \quad \text { and } \quad Z_{-}=(-\infty, 0] \times S^{1} .
$$

Then writing $u$ near the puncture in cylindrical coordinates $(s, t)$, for $|s|$ sufficiently large, it satisfies an asymptotic formula of the form

$$
u \circ \varphi(s, t)=\exp _{(T s, x(T t))} h(s, t) \in E_{ \pm} .
$$

Here $T>0$ is a constant, $x: \mathbb{R} \rightarrow M_{ \pm}$is a $T$-periodic orbit of $X_{ \pm}$, the exponential map is defined with respect to any $\mathbb{R}$-invariant metric on $\mathbb{R} \times M_{ \pm}, h(s, t) \in \xi_{x(T t)}$ goes to 0 uniformly in $t$ as $s \rightarrow \pm \infty$ and $\varphi: Z_{ \pm} \rightarrow Z_{ \pm}$is a smooth embedding such that

$$
\varphi(s, t)-\left(s+s_{0}, t+t_{0}\right) \rightarrow 0
$$

as $s \rightarrow \pm \infty$ for some constants $s_{0} \in \mathbb{R}, t_{0} \in S^{1}$. We will denote by $\gamma_{z}$ the $T$-periodic orbit parametrized by $x$, and call it the asymptotic orbit of $u$ at the puncture $z$. With this asymptotic behavior in mind, it is convenient to think of $(\dot{\Sigma}, j)$ as a Riemann surface with cylindrical ends, and we will sometimes refer to neighborhoods of the punctures as ends of $\dot{\Sigma}$. As is well known (cf. [Hof93], [HWZ96]), the asymptotically cylindrical holomorphic curves in $(W, J)$ are precisely those which satisfy a certain finite energy condition, though we will not need this fact here.

Denote by $\mathcal{M}:=\mathcal{M}(J, L)$ the moduli space of equivalence classes of asymptotically cylindrical $J$-holomorphic curves in $W$ with boundary on $L$; here an equivalence class is defined by the data $(\Sigma, j, \Gamma, u)$ where $\Gamma$ is considered to be an ordered set, and we define $(\Sigma, j, \Gamma, u) \sim\left(\Sigma^{\prime}, j^{\prime}, \Gamma^{\prime}, u^{\prime}\right)$ if there exists a biholomorphic map $\varphi:(\Sigma, j) \rightarrow\left(\Sigma^{\prime}, j^{\prime}\right)$ taking $\Gamma$ to $\Gamma^{\prime}$ with the ordering preserved, such that $u=u^{\prime} \circ \varphi$. We shall often abuse notation and write $u \in \mathcal{M}$ or $(\Sigma, j, \Gamma, u) \in \mathcal{M}$ when we mean $[(\Sigma, j, \Gamma, u)] \in \mathcal{M}$. The moduli space has a natural topology defined by $C_{\text {loc }}^{\infty}$-convergence on $\dot{\Sigma}$ and uniform convergence up to the ends. For any $u \in \mathcal{M}$, denote by $\mathcal{M}_{u}$ the connected component of $\mathcal{M}$ containing $u$.

It is often interesting to consider subspaces of $\mathcal{M}$ defined by imposing constraints on the asymptotic behavior at some of the punctures.

Definition 1.1. For a given punctured surface $\dot{\Sigma}=\Sigma \backslash\left(\Gamma^{+} \cup \Gamma^{-}\right)$, let $\mathbf{c}$ denote a choice of periodic orbit $\gamma_{z}^{\mathbf{c}}$ in $M_{ \pm}$for some subset of punctures $z \in \Gamma^{ \pm}$. We call $\mathbf{c}$ a choice of asymptotic constraints, ${ }^{4}$ and refer to each puncture $z$ for which $\mathbf{c}$ specifies an orbit $\gamma_{z}^{\mathbf{c}}$ as a constrained puncture.

\footnotetext{
${ }^{4}$ One can impose more stringent constraints as well, e.g. on the rate at which $u$ converges to its asymptotic orbits; such constraints are treated in [Wenb], [Wena]. Another possibility is to allow marked points that map to specified points in the image, perhaps with cusps of prescribed order, as in [Bar00], [Fra05]. We omit all these possibilities here for the sake of brevity.
} 
For any choice of domain $\dot{\Sigma}$ and asymptotic constraints c, we can consider the constrained moduli space

$$
\mathcal{M}^{\mathbf{c}} \subset \mathcal{M}
$$

consisting of curves $u: \dot{\Sigma} \rightarrow W$ that approach the specified orbit $\gamma_{z}^{\mathbf{c}}$ at each of the constrained punctures $z \in \Gamma$, and arbitrary orbits at the unconstrained punctures. The constraints define another partition of $\Gamma$,

$$
\Gamma=\Gamma_{C} \cup \Gamma_{U}
$$

into the sets of constrained and unconstrained punctures respectively. The positive and negative subsets within each of these will be denoted by $\Gamma_{C}^{ \pm}$and $\Gamma_{U}^{ \pm}$.

If the asymptotic orbits of $u$ are all Morse-Bott, then the so-called virtual dimension of $\mathcal{M}_{u}^{\mathbf{c}}$ is given by the Fredholm index

$$
\operatorname{ind}(u ; \mathbf{c})=(n-3) \chi(\dot{\Sigma})+2 c_{1}^{\Phi}\left(u^{*} T W\right)+\mu^{\Phi}(u ; \mathbf{c}),
$$

where $c_{1}^{\Phi}\left(u^{*} T W\right)$ is the relative first Chern number of $\left(u^{*} T W, J\right) \rightarrow \dot{\Sigma}$ with respect to a suitable choice of trivialization $\Phi$ along the ends and boundary, and $\mu^{\Phi}(u$; $\mathbf{c})$ is a sum of Conley-Zehnder indices of the asymptotic orbits and a Maslov index at the boundary with respect to $\Phi$; a precise definition will be given in $\$ 3.2$.

As we shall review in more detail in $\S 3$, the nonlinear Cauchy-Riemann equation can be expressed as a smooth section of a Banach space bundle

$$
\bar{\partial}_{J}: \mathscr{B} \rightarrow \mathcal{E}, \quad(j, u) \mapsto T u+J \circ T u \circ j,
$$

such that a neighborhood of any non-constant $(\Sigma, j, \Gamma, u)$ in $\mathcal{M}^{\mathbf{c}}$ is in one-to-one correspondence with $\bar{\partial}_{J}^{-1}(0) / \operatorname{Aut}(\dot{\Sigma}, j)$, where the group $\operatorname{Aut}(\dot{\Sigma}, j)$ of biholomorphic maps $(\Sigma, j) \rightarrow(\Sigma, j)$ fixing $\Gamma$ acts on pairs $\left(j^{\prime}, u^{\prime}\right) \in \bar{\partial}_{J}^{-1}(0)$ by

$$
\varphi \cdot\left(j^{\prime}, u^{\prime}\right)=\left(\varphi^{*} j^{\prime}, u^{\prime} \circ \varphi\right) .
$$

It is then standard to say that $(\Sigma, j, \Gamma, u) \in \mathcal{M}$ is regular if it represents a transverse intersection with the zero-section, i.e. the linearization

$$
D \bar{\partial}_{J}(j, u): T_{(j, u)} \mathcal{B} \rightarrow \mathcal{E}_{(j, u)}
$$

is surjective. We will give a precise definition in $\$ 3.2$ once the functional analytic setup is in place. Observe that if $u: \dot{\Sigma} \rightarrow W$ is not constant, then the action of $\operatorname{Aut}(\dot{\Sigma}, j)$ induces a natural inclusion of its Lie algebra aut $(\dot{\Sigma}, j)$ into ker $D \bar{\partial}_{J}(j, u)$. For the sake of completeness, we will present in $\S 3.2$ a proof of the following standard folk theorem:

Theorem 0. Assume $u:(\dot{\Sigma}, j) \rightarrow(W, J)$ is a non-constant curve in $\mathcal{M}^{\mathbf{c}}$ with only Morse-Bott asymptotic orbits. If $u$ is regular, then a neighborhood of $u$ in $\mathcal{M}^{\mathbf{c}}$ 
naturally admits the structure of a smooth orbifold of dimension $\operatorname{ind}(u ; \mathbf{c})$, whose isotropy group at $u$ is

$$
\operatorname{Aut}(u):=\{\varphi \in \operatorname{Aut}(\dot{\Sigma}, j) \mid u=u \circ \varphi\},
$$

and there is a natural isomorphism

$$
T_{u} \mathcal{M}^{\mathbf{c}}=\operatorname{ker} D \bar{\partial}_{J}(j, u) / \operatorname{aut}(\dot{\Sigma}, j) .
$$

In particular, regularity implies that $\mathcal{M}^{\mathbf{c}}$ is a manifold near $u$ if $u$ is somewhere injective, and in general the isotropy group for an orbifold singularity has order bounded by the covering number of $u$. Note that in contrast to the standard theory of $J$-holomorphic curves (cf. [MS04]), we shall in this paper be especially interested in cases where $u$ achieves regularity despite being multiply covered, so the moduli space is smooth but may be an orbifold rather than a manifold.

In the case $\operatorname{dim} W=4$, another number that turns out to play an important role is the so-called normal first Chern number $c_{N}(u ; \mathbf{c}) \in \frac{1}{2} \mathbb{Z}$, which can be defined most simply via the formula

$$
2 c_{N}(u ; \mathbf{c})=\operatorname{ind}(u ; \mathbf{c})-2+2 g+\# \Gamma_{0}(\mathbf{c})+\# \pi_{0}(\partial \Sigma) .
$$

Here $g$ is the genus of $\Sigma$ and $\Gamma_{0}(\mathbf{c}) \subset \Gamma$ is the subset of punctures for which the asymptotic orbit has even Conley-Zehnder index (this is the correct definition if all orbits are nondegenerate; in the Morse-Bott case the definition is more complicated and may depend on the asymptotic constraints, see \$3.2). We will be able to give a better motivated definition in $\$ 3.5$ using the linear theory in $\S 2$, but for now, the significance of $c_{N}(u ; \mathbf{c})$ can be illustrated by considering the case where $\Sigma$ is closed and $\Gamma=\emptyset$. Then a combination of (1.1) and (1.2) yields the relation $c_{N}(u ; \mathbf{c})=$ $c_{1}\left(u^{*} T W\right)-\chi(\Sigma)$, so $c_{N}(u ; \mathbf{c})$ is the first Chern number of the normal bundle if $u$ is immersed. This is the appropriate philosophical interpretation of $c_{N}(u ; \mathbf{c})$ in general, as will become obvious from further considerations.

As a final piece of preparation, note that since a non-constant holomorphic curve $u: \dot{\Sigma} \rightarrow W$ is necessarily immersed near the ends, it can have at most finitely many critical points. Indeed, as we will review in $\S 3.3$, the bundle $u^{*} T W \rightarrow \dot{\Sigma}$ admits a natural holomorphic structure such that the section

$$
d u \in \Gamma\left(\operatorname{Hom}_{\mathbb{C}}\left(T \dot{\Sigma}, u^{*} T W\right)\right)
$$

is holomorphic; its critical points are thus isolated and have positive order, which we denote by $\operatorname{ord}(d u ; z)$ for any $z \in \operatorname{Crit}(u)$. The quantity

$$
Z(d u):=\sum_{z \in d u^{-1}(0) \cap \operatorname{int} \dot{\Sigma}} \operatorname{ord}(d u ; z)+\frac{1}{2} \sum_{z \in d u^{-1}(0) \cap \partial \Sigma} \operatorname{ord}(d u ; z)
$$

is therefore a finite nonnegative half-integer (or integer if $\partial \Sigma=\emptyset$ ), and it equals zero if and only if $u$ is immersed. 
1.2. Local and global transversality results. We now state the main result of this paper. The following will be a convenient piece of shorthand notation: if $\partial \Sigma \neq \emptyset$, then for given constants $c \in \mathbb{R}$ and $G \geq 0$, define the nonnegative integer

$$
\begin{array}{r}
K(c, G)=\min \{k+\ell \mid \\
k, \ell \text { nonnegative integers, } \\
k \leq G \text { and } 2 k+\ell>2 c\} .
\end{array}
$$

If $\partial \Sigma=\emptyset$ we modify this definition slightly by requiring the integer $\ell$ to be even. Note that in most applications known to the author, it will turn out that $c<0$, so $K(c, G)=0$.

Theorem 1. Suppose $\operatorname{dim} W=4$ and $(\Sigma, j, \Gamma, u) \in \mathcal{M}^{\mathbf{c}}$ is a non-constant curve with only Morse-Bott asymptotic orbits. If

$$
\operatorname{ind}(u ; \mathbf{c})>c_{N}(u ; \mathbf{c})+Z(d u),
$$

then $u$ is regular. Moreover, when this condition is not satisfied, we have the following bounds on the dimension of $\operatorname{ker} D \bar{\partial}_{J}(j, u):$ if $\operatorname{ind}(u ; \mathbf{c}) \leq 2 Z(d u)$, then

$$
\begin{aligned}
2 Z(d u) & \leq \operatorname{dim}\left(\operatorname{ker} D \bar{\partial}_{J}(j, u) / \operatorname{aut}(\dot{\Sigma}, j)\right) \\
& \leq 2 Z(d u)+K\left(c_{N}(u ; \mathbf{c})-Z(d u), \# \Gamma_{0}(\mathbf{c})\right),
\end{aligned}
$$

and if $2 Z(d u) \leq \operatorname{ind}(u ; \mathbf{c})$, then

$$
\begin{aligned}
\operatorname{ind}(u ; \mathbf{c}) & \leq \operatorname{dim}\left(\operatorname{ker} D \bar{\partial}_{J}(j, u) / \operatorname{aut}(\dot{\Sigma}, j)\right) \\
& \leq \operatorname{ind}(u ; \mathbf{c})+K\left(c_{N}(u ; \mathbf{c})+Z(d u)-\operatorname{ind}(u ; \mathbf{c}), \# \Gamma_{0}(\mathbf{c})\right) .
\end{aligned}
$$

Remark 1.2. Plugging in the definition of $c_{N}(u ; \mathbf{c})$ and the index formula, the condition (1.5) is equivalent to

$$
\operatorname{ind}(u ; \mathbf{c})>2 g+\# \Gamma_{0}(\mathbf{c})+\# \pi_{0}(\partial \Sigma)-2+2 Z(d u),
$$

or

$$
2 c_{1}^{\Phi}\left(u^{*} T W\right)+\mu^{\Phi}(u ; \mathbf{c})+\# \Gamma_{1}(\mathbf{c})>2 Z(d u),
$$

where $\Gamma_{1}(\mathbf{c}):=\Gamma \backslash \Gamma_{0}(\mathbf{c})$. These are direct generalizations of the criteria in [HLS97], [Wen05], [IS99].

Remark 1.3. There is an important special case of the dimension bound which we will

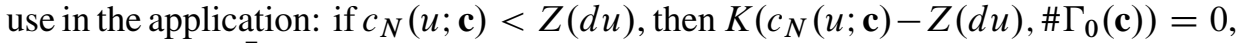
thus dim $\operatorname{ker}\left(D \bar{\partial}_{J}(j, u)\right)$ becomes $2 Z(d u)$, its smallest possible value.

Results of this type have been used previously for a variety of applications, including disk filling and deformation arguments in contact 3-manifolds [Hof93], 
[HWZ95b], [Wen08], and the symplectic isotopy problem [She01], [Sik03]. In the last section of this paper, we will use our generalization to prove a somewhat surprising global structure theorem for certain geometrically natural moduli spaces of holomorphic curves in 4-dimensional symplectic cobordisms.

To motivate this, consider for a moment the case of a closed holomorphic curve $u: \Sigma \rightarrow W$ that satisfies the criterion ind $(u)>c_{N}(u)+Z(d u)$. We know then that $\mathcal{M}$ is smooth in some neighborhood of $u$, but ideally one would like to know that the entire connected component $\mathcal{M}_{u}$ is smooth. In general this will not be true, as other curves in $\mathcal{M}_{u}$ may have more critical points and thus fail to satisfy the criterion. One favorite way to evade this issue is by assuming that $u$ is embedded: then the adjunction formula (cf. [MS04]) guarantees that all somewhere injective curves $u^{\prime} \in \mathcal{M}_{u}$ are also embedded, hence $Z\left(d u^{\prime}\right)=0$ and the criterion is satisfied. The catch is that unless one imposes additional restrictive conditions on the homology class $[u] \in \mathrm{H}_{2}(W)$, not every curve in $\mathcal{M}_{u}$ need be somewhere injective: a sequence of embedded curves may converge to a branched cover, which will not always be regular since its branch points are critical. Thus $\mathcal{M}_{u}$ may fail to be globally smooth if it contains branched covers, and there is no way to avoid this in general. ${ }^{5}$

The surprising fact is that if we impose an additional, rather natural intersectiontheoretic condition on $u$, then the multiple covers that arise turn out to be "harmless": even the multiple covers are regular, and $\mathcal{M}_{u}$ is thus globally smooth. The condition in question arises from the study of $J$-holomorphic foliations: in particular, we focus on punctured embedded curves $u: \dot{\Sigma} \rightarrow W$ that exist in 1- or 2-dimensional families (with respect to some constraints $\mathbf{c}$ ) and have the property of never intersecting their neighbors, i.e. these families foliate either an open set or a hypersurface containing $u(\dot{\Sigma}) \subset W$. A complete characterization of such curves is given in [Wenb] and will be reviewed in $\S 4$; we refer to them as stable, nicely embedded curves. If $u$ is such a curve, then it automatically satisfies the criterion of Theorem 1, thus the local structure of $\mathcal{M}_{u}^{\mathbf{c}}$ near $u$ is well understood, but one still has the global question:

Question. Can a sequence of stable, nicely embedded curves converge to a multiple cover?

If the answer is no, then $\mathcal{M}_{u}^{\mathbf{c}}$ is a smooth manifold, and we shall show that this is indeed the case whenever $W$ is an $\mathbb{R}$-invariant symplectization (with generic $J$ ) or a closed symplectic manifold. In general, it turns out that multiple covers can appear, but only if they are immersed, in which case the regularity criterion is still satisfied. The proof of this fact will make use of our transversality arguments for nonimmersed curves, establishing in effect that any component of $\mathcal{M}^{\mathbf{c}}$ containing such a non-immersed multiple cover can consist only of multiple covers. The result is:

\footnotetext{
${ }^{5}$ Note that multiply covered curves also pose a problem in the standard transversality theory, but for completely different reasons.
} 
Theorem 2. For generic $J$, if $u \in \mathcal{M}^{\mathbf{c}}$ is a stable, nicely embedded curve, then every curve in $\mathcal{M}_{u}^{\mathbf{c}}$ is regular: in particular $\mathcal{M}_{u}^{\mathbf{c}}$ naturally admits the structure of a smooth orbifold of dimension $\operatorname{ind}(u ; \mathbf{c}) \in\{1,2\}$, with only isolated singularities. Moreover, all curves in $\mathcal{M}_{u}^{\mathbf{c}}$ are embedded except for a discrete subset, consisting of unbranched multiple covers over stable, nicely embedded index 0 curves, and the images of any two curves in $\mathcal{M}_{u}^{\mathbf{c}}$ are either identical or disjoint.

This will follow from a more general result (Theorem 4) proved in $\S 4$, which applies also to parametrized moduli spaces under a generic homotopy of almost complex structures. As a simple corollary, we observe the two aforementioned cases where the answer to the question posed above is no:

Corollary 1.4. For the curve $u: \dot{\Sigma} \rightarrow W$ in Theorem 2, suppose that either

- $\dot{\Sigma}$ is a closed Riemann surface (without punctures), or

- $(W, J)=(\mathbb{R} \times M, \tilde{J})$ is the symplectization of a 3-manifold with stable Hamiltonian structure $\mathscr{H}=(X, \xi, \omega, J)$, where $J$ is generic.

Then every curve in $\mathcal{M}_{u}^{\mathbf{c}}$ is embedded, thus $\mathcal{M}_{u}^{\mathbf{c}}$ is a manifold.

Proof. For the $\mathbb{R}$-invariant symplectization $(\mathbb{R} \times M, \tilde{J})$, a multiple cover $u=v \circ \varphi$ would require a somewhere injective curve $v$ of index 0 , which does not exist if $J$ is generic. The reasoning in the closed case is different: it depends on the fact that, as we shall show in $\S 4.3$, stable nicely embedded curves always have genus zero. Then $\varphi$ must be a holomorphic map $S^{2} \rightarrow S^{2}$ with no branch points, contradicting the Riemann-Hurwitz formula.

Unbranched multiple covers can and do appear in general if $\dot{\Sigma}$ has punctures and $(W, J)$ is a non-cylindrical manifold, e.g. a nontrivial symplectic cobordism. We will show an example at the end of $\S 4$, where the resulting collection of curves actually foliates $W$.

The phenomenon illustrated by Theorem 2 contrasts with the more general study of holomorphic curves, e.g. in Symplectic Field Theory, where transversality can only be achieved in general by abstract perturbations. Such perturbations usually destroy many of the nice geometric properties of holomorphic curves - such as positivity of intersections - but the philosophy here is that for curves that are especially nice in some geometric sense, precisely these nice properties make abstract perturbations unnecessary. In particular, the theorem is part of a larger program outlined in [Wena], to prove that the compactified moduli spaces of curves that can occur in foliations always have a nice global structure: in principle, after proving a suitable compactness theorem for this "nice" class of curves, transversality should always follow "for free". Such results are necessary tools in the general theory of $J$-holomorphic foliations, as one would like to prove that these foliations can always be carried through under 
various types of homotopies and stretching arguments. The situation is already well understood in the $\mathbb{R}$-invariant case due to [Wena], and Theorem 2 may be seen as a partial result in the direction of generalizing that compactness theorem to symplectic cobordisms. (See Example 4.22 and Remark 4.23 for an idea of what such a generalization might look like.)

1.3. Outline of the proofs. The technical backbone of Theorem 1 is the analysis of the normal Cauchy-Riemann operator $\boldsymbol{D}_{u}^{N}$ associated to any holomorphic curve $u: \dot{\Sigma} \rightarrow W$. As we will recall, this is well defined even if $u$ has critical points, because there always exists a splitting

$$
u^{*} T W=T_{u} \oplus N_{u}
$$

such that $\left(T_{u}\right)_{z}$ is the image of $T_{z} \dot{\Sigma}$ under $d u$ at all regular points $z$. The domain of $D_{u}^{N}$ is then a space of sections of $N_{u}$, a complex line bundle. We describe the required linear theory of such operators in $\S 2$, giving criteria that guarantee surjectivity of $\boldsymbol{D}_{u}^{N}$ as well as bounds on the dimension of its kernel.

The next step is then to relate the operator $\boldsymbol{D}_{u}^{N}$ to the nonlinear problem. In the immersed case, the traditional approach (cf. [HLS97], [HWZ99]) is to set up the nonlinear problem to detect $J$-invariant maps that can be expressed as sections of the normal bundle of a given solution $u$, in which case the linearization is equivalent to $\boldsymbol{D}_{u}^{N}$. This is no longer possible when $u$ has critical points; Ivashkovich and Shevchishin in [IS99] dealt with this difficulty by replacing the normal bundle with a normal sheaf and proving that $u$ is regular if and only if $\boldsymbol{D}_{u}^{N}$ is surjective. Our approach takes some inspiration from theirs but is less algebraic and more analytical in flavor, as we avoid any reference to sheaves and exact sequences in favor of Banach space splittings and Fredholm operators. Unlike [HLS97], [HWZ99], we treat the nonlinear problem in the way that is standard for arbitrary dimensions, as a section

$$
\bar{\partial}_{J}: \mathcal{T} \times \mathscr{B} \rightarrow \mathcal{E}
$$

of a suitable Banach space bundle, where $\mathscr{B}$ is a (globally defined) Banach manifold of maps $\dot{\Sigma} \rightarrow W$ (including reparametrizations) and $\mathcal{T}$ is a (locally defined) finite dimensional space of complex structures parametrizing on open subset in the Teichmüller space of $\dot{\Sigma}$. We will use the splitting $u^{*} T W=T_{u} \oplus N_{u}$ and some properties of the standard Cauchy-Riemann operator on $\Gamma(T \dot{\Sigma})$ to give a precise relation (Theorem 3) between the kernels and images of $D \bar{\partial} J(j, u)$ and $\boldsymbol{D}_{u}^{N}$ in arbitrary dimensions. A consequence is the fact that each of these operators is surjective if and only if the other is.

As for the proof of Theorem 2: assume $u_{n}$ is a sequence of stable, nicely embedded curves converging to a multiple cover $u=v \circ \varphi$, where $v$ is somewhere injective. We observe first that the embedded curves $u_{n}$ necessarily satisfy the criterion of 
Theorem 1, so this will remain true for the limit $u$ unless it acquires critical points. The main task then is to show that $u$ is immersed, and the kernel bounds in Theorem 1 for non-immersed curves turn out to be a useful tool in proving this. The first step is to show that the underlying simple curve $v$ is embedded and has index 0 : this follows by a careful application of the intersection theory of punctured holomorphic curves, which we review at the beginning of $\S 4$. Note that this is the only point in the argument at which we assume $J$ to be generic: it is necessary to obtain a lower bound on the index of $v$ and thus on its related intersection invariants, but it will not be required in proving transversality for $u$. With this established, critical points of $u$ arise only from branch points of the cover $\varphi$, hence $Z(d u)=Z(d \varphi)$, i.e. the ramification number of $\varphi$. Now the dimension bound in Theorem 1 turns out to imply that a neighborhood of $u$ in $\mathcal{M}_{u}^{\mathbf{c}}$ "lives inside a space of dimension at most $2 Z(d u)$ "; we will make this statement precise later using the implicit function theorem. But if $Z(d u)>0$, then the space of holomorphic branched covers homotopic to $\varphi$ is nontrivial and has precisely this dimension, which yields a $2 Z(d u)$-dimensional smooth submanifold of $\mathcal{M}_{u}^{\mathbf{c}}$ containing $u$. It follows that this describes a neighborhood of $u$ in $\mathcal{M}_{u}^{\mathbf{c}}$, so any sequence of curves converging to $u$ must then have the form $u_{n}=v \circ \varphi_{n}$, i.e. they are all multiple covers with the same image, and this is a contradiction.

Acknowledgments. Many thanks to Denis Auroux, Kai Cieliebak, Oliver Fabert, Helmut Hofer, Sam Lisi, Klaus Mohnke, Sewa Shevchishin and Richard Siefring for useful conversations.

\section{Cauchy-Riemann type operators on bundles}

2.1. Generalities. Let $(\Sigma, j)$ be a compact Riemann surface with genus $g, m \geq$ 0 boundary components, and a finite set of positive/negative interior punctures $\Gamma=$ $\Gamma^{+} \cup \Gamma^{-} \subset$ int $\Sigma$, with the corresponding punctured surface denoted by $\dot{\Sigma}=\Sigma \backslash \Gamma$. Regarding $\dot{\Sigma}$ as a surface with cylindrical ends $\left\{U_{z}\right\}_{z \in \Gamma^{ \pm}}$biholomorphic to the halfcylinders $Z_{ \pm}$, it admits a natural compactification $\bar{\Sigma}$ obtained by replacing $[0, \infty) \times S^{1}$ by $[0, \infty] \times S^{1}$ and $(-\infty, 0] \times S^{1}$ by $[-\infty, 0] \times S^{1}$. The compactified space is naturally a topological 2-manifold with boundary

$$
\partial \bar{\Sigma}=\partial \Sigma \sqcup \bigcup_{z \in \Gamma} \delta_{z}
$$

where for each $z \in \Gamma^{ \pm}, \delta_{z} \cong\{ \pm \infty\} \times S^{1}$ denotes the corresponding "circle at infinity". Note that in making this definition we have chosen cylindrical coordinates $(s, t) \in Z_{ \pm}$over each end $\left\{U_{z}\right\}_{z \in \Gamma^{ \pm}}$, and we will continue to use these coordinates whenever convenient. The definitions of $\bar{\Sigma}$ and $\delta_{z}$ do not depend on this choice, 
and in fact the resulting identification of each $\delta_{z}$ with $S^{1}=\mathbb{R} / \mathbb{Z}$ is unique up to a constant shift.

Let $E \rightarrow \bar{\Sigma}$ be a complex vector bundle of rank $n$ whose restriction to $\dot{\Sigma}$ and each of the circles $\delta_{z}$ has a smooth structure. Assume moreover that $E$ is given a Hermitian structure over each end $U_{z}$. By an admissible trivialization of $E$ near $z \in \Gamma^{ \pm}$, we mean a smooth unitary bundle isomorphism $\Phi: E \mid u_{z} \rightarrow Z_{ \pm} \times \mathbb{R}^{2 n}$ (where $\mathbb{R}^{2 n}$ is identified with $\mathbb{C}^{n}$ ), which covers the coordinate map $U_{z} \rightarrow Z_{ \pm}$ and extends continuously to a smooth unitary trivialization $\left.E\right|_{\delta_{z}} \rightarrow S^{1} \times \mathbb{R}^{2 n}$. An asymptotic operator at $z \in \Gamma$ is then a bounded real linear operator

$$
\boldsymbol{A}_{z}: H^{1}\left(\left.E\right|_{\delta_{z}}\right) \rightarrow L^{2}\left(E_{\delta_{z}}\right)
$$

whose expression with respect to any admissible trivialization takes the form

$$
H^{1}\left(S^{1}, \mathbb{R}^{2 n}\right) \rightarrow L^{2}\left(S^{1}, \mathbb{R}^{2 n}\right), \quad \eta \mapsto-J_{0} \dot{\eta}-S \eta ;
$$

here $J_{0}=i$ is the standard complex structure on $\mathbb{R}^{2 n}=\mathbb{C}^{n}$ and $S=S(t)$ is any smooth loop of symmetric $2 n$-by- $2 n$ matrices. This defines an unbounded self-adjoint operator on the complexification of $L^{2}\left(\left.E\right|_{\delta_{z}}\right)$. We say that $\boldsymbol{A}_{z}$ is nondegenerate if its spectrum $\sigma\left(\boldsymbol{A}_{z}\right)$ does not contain 0 .

Define the standard $\bar{\partial}$-operator for smooth functions on $\dot{\Sigma}$ by

$$
\bar{\partial}: C^{\infty}(\dot{\Sigma}, \mathbb{C}) \rightarrow \Omega^{0,1}(\dot{\Sigma}), \quad f \mapsto d f+i d f \circ j .
$$

For any two complex vector bundles $E$ and $E^{\prime}$ over the same base, we denote by $\operatorname{Hom}_{\mathbb{C}}\left(E, E^{\prime}\right)$ and $\overline{\operatorname{Hom}}_{\mathbb{C}}\left(E, E^{\prime}\right)$ the corresponding bundles of complex linear and antilinear maps $E \rightarrow E^{\prime}$ respectively. There are also the corresponding endomorphism bundles End $\mathbb{C}_{\mathbb{C}}(E):=\operatorname{Hom}_{\mathbb{C}}(E, E)$ and $\overline{\operatorname{End}}_{\mathbb{C}}(E):=\overline{\operatorname{Hom}}_{\mathbb{C}}(E, E)$.

Definition 2.1. A (smooth, real linear) Cauchy-Riemann type operator on $E$ is a first-order linear differential operator

$$
\boldsymbol{D}: \Gamma\left(\left.E\right|_{\dot{\Sigma}}\right) \rightarrow \Gamma\left(\overline{\operatorname{Hom}}_{\mathbb{C}}\left(T \dot{\Sigma},\left.E\right|_{\dot{\Sigma}}\right)\right)
$$

such that for every smooth section $v: \dot{\Sigma} \rightarrow E$ and smooth function $f: \dot{\Sigma} \rightarrow \mathbb{R}$,

$$
\boldsymbol{D}(f v)=(\bar{\partial} f) v+f(\boldsymbol{D} v) .
$$

Given an asymptotic operator $\boldsymbol{A}_{z}$ at $z \in \Gamma^{ \pm}$, we will say that $\boldsymbol{D}$ is asymptotic to $\boldsymbol{A}_{z}$ if its expression in an admissible trivialization $\Phi$ near $z$ takes the form

$$
(\boldsymbol{D} v)(s, t)=\partial_{s} v(s, t)+J_{0} \partial_{t} v(s, t)+S(s, t) v(s, t),
$$

where $S(s, t)$ is a smooth family of real-linear transformations on $\mathbb{R}^{2 n}$ which converges uniformly as $s \rightarrow \pm \infty$ to a smooth loop of symmetric matrices $S(t)$, such that

$$
-J_{0} \frac{d}{d t}-S(t)
$$

is the coordinate expression for $\boldsymbol{A}_{z}$ with respect to $\Phi$. 
Define the Banach space $W^{k, p}(E)$ to consist of sections $v: \dot{\Sigma} \rightarrow E$ of class $W_{\text {loc }}^{k, p}$ such that in any choice of admissible trivialization near each puncture $z \in \Gamma^{ \pm}$, the corresponding map $Z_{ \pm} \rightarrow \mathbb{R}^{2 n}$ is of class $W^{k, p}$. If $\left.\ell \subset E\right|_{\partial \Sigma}$ is a smooth totally real submanifold, define the subspace

$$
W_{\ell}^{k, p}(E)=\left\{v \in W^{k, p}(E) \mid v(\partial \Sigma) \subset \ell\right\} .
$$

Observe that $\overline{\operatorname{Hom}}_{\mathbb{C}}(T \dot{\Sigma}, E)$ also admits a natural extension over $\bar{\Sigma}$, and the combination of the coordinates $(s, t)$ with the trivialization $\Phi$ near $z \in \Gamma$ also gives rise to a trivialization of $\overline{\operatorname{Hom}}_{\mathbb{C}}(T \dot{\Sigma}, E)$. Using this we can define the Banach spaces $W^{k, p}\left(\overline{\operatorname{Hom}}_{\mathbb{C}}(T \dot{\Sigma}, E)\right)$. We will generally write $W^{0, p}$ as $L^{p}$.

Now fix a smooth totally real subbundle $\left.\ell \subset E\right|_{\partial \Sigma}$, and asymptotic operators $\boldsymbol{A}_{z}$ for each $z \in \Gamma$, denoting the collection of all these operators by $\boldsymbol{A}_{\Gamma}$. Let $\boldsymbol{D}$ be a Cauchy-Riemann type operator that is asymptotic to $\boldsymbol{A}_{z}$ for each $z \in \Gamma$. We will then be interested in the bounded linear operator

$$
\boldsymbol{D}: W_{\ell}^{1, p}(E) \rightarrow L^{p}\left(\overline{\operatorname{Hom}}_{\mathbb{C}}(T \dot{\Sigma}, E)\right) .
$$

This is a Fredholm operator if all the $\boldsymbol{A}_{z}$ are nondegenerate, and its index is determined by a variety of topological quantities which we shall recall next.

Fix a set of admissible trivializations near each puncture $z \in \Gamma$ as well as smooth complex trivializations of $\left.E\right|_{\partial \Sigma}$, denoting the collection of all these choices by $\Phi$. One can then define the relative first Chern number $c_{1}^{\Phi}(E) \in \mathbb{Z}$. If $E$ is a line bundle, then $c_{1}^{\Phi}(E)$ is defined simply by counting zeroes of a generic smooth section $\dot{\Sigma} \rightarrow E$ that extends continuously over $\bar{\Sigma}$ and is a nonzero constant with respect to $\Phi$ on $\partial \bar{\Sigma}$. For higher rank bundles, $c_{1}^{\Phi}(E)$ can be defined axiomatically via the direct sum property and the assumption that it matches the ordinary first Chern number if $\dot{\Sigma}$ is closed.

For each connected component $C \subset \partial \Sigma$, the totally real subbundle $\left.\left.\ell\right|_{C} \subset E\right|_{C}$ has a Maslov index $\mu^{\Phi}\left(\left.E\right|_{C},\left.\ell\right|_{C}\right)$, and we shall denote the sum of these by $\mu^{\Phi}(E, \ell)$.

Finally for each puncture $z \in \Gamma^{ \pm}$, the asymptotic operator $\boldsymbol{A}_{z}$, expressed as $-J_{0} \partial_{t}-S(t)$ with respect to $\Phi$, gives rise to a linear Hamiltonian flow in $\mathbb{R}^{2 n}$ via the equation

$$
\dot{\eta}(t)=J_{0} S(t) \eta(t) .
$$

If $\boldsymbol{A}_{z}$ is nondegenerate, then the resulting path of symplectic matrices $\Psi(t) \in \operatorname{Sp}(n)$ ends at a matrix $\Psi(1)$ which does not have 1 as an eigenvalue, so it has a well defined Conley-Zehnder index which we denote by $\mu_{\mathrm{CZ}}^{\Phi}\left(\boldsymbol{A}_{z}\right)$. All of this together allows us to define the total Maslov index

$$
\mu^{\Phi}\left(E, \ell, \boldsymbol{A}_{\Gamma}\right):=\mu^{\Phi}(E, \ell)+\sum_{z \in \Gamma^{+}} \mu_{\mathrm{CZ}}^{\Phi}\left(\boldsymbol{A}_{z}\right)-\sum_{z \in \Gamma^{-}} \mu_{\mathrm{CZ}}^{\Phi}\left(\boldsymbol{A}_{z}\right) .
$$


The Fredholm index of $\boldsymbol{D}$ is then given by the following generalization of the Riemann-Roch formula:

$$
\operatorname{ind}(\boldsymbol{D})=n \chi(\dot{\Sigma})+2 c_{1}^{\Phi}(E)+\mu^{\Phi}\left(E, \ell, \boldsymbol{A}_{\Gamma}\right) .
$$

This follows from the formula for the case $\partial \Sigma=\emptyset$ proved in [Sch95], together with a gluing/doubling argument; cf. [Wen05]. Note that all dependence on $\Phi$ in the right hand side of (2.1) cancels out.

Let us briefly review the useful generalization of the above that arises by considering Banach spaces with exponential weights. Pick numbers $\boldsymbol{\delta}_{z} \in \mathbb{R}$ for each $z \in \Gamma$ and denote the collection of these by $\boldsymbol{\delta}_{\Gamma}=\left\{\boldsymbol{\delta}_{z}\right\}_{z \in \Gamma}$. Then we define

$$
W^{k, p, \boldsymbol{\delta}_{\Gamma}}(E)
$$

to be the space of $W_{\text {loc }}^{k, p}$ sections $v: \dot{\Sigma} \rightarrow E$ such that in an admissible trivialization near each $z \in \Gamma^{ \pm}$, the function $Z_{ \pm} \rightarrow \mathbb{R}^{2 n},(s, t) \mapsto e^{ \pm \delta_{z} s} v(s, t)$ is of class $W^{k, p}$. This imposes an exponential decay condition at each puncture where $\boldsymbol{\delta}_{z}>0$, or a bound on exponential growth if $\boldsymbol{\delta}_{z}<0$. There are now obvious definitions for the spaces $W_{\ell}^{1, p, \delta_{\Gamma}}(E)$ and $L^{p, \delta_{\Gamma}}\left(\overline{\operatorname{Hom}}_{\mathbb{C}}(T \dot{\Sigma}, E)\right)$, so that the Cauchy-Riemann type operator $\boldsymbol{D}$ defines a bounded linear map

$$
\boldsymbol{D}: W_{\ell}^{1, p, \boldsymbol{\delta}_{\Gamma}}(E) \rightarrow L^{p, \boldsymbol{\delta}_{\Gamma}}\left(\overline{\operatorname{Hom}}_{\mathbb{C}}(T \dot{\Sigma}, E)\right) .
$$

It is simple to show (cf. [HWZ99], [Wenb]) that this map is conjugate to another Cauchy-Riemann operator $\boldsymbol{D}_{\boldsymbol{\delta}_{\Gamma}}: W_{\ell}^{1, p}(E) \rightarrow L^{p}\left(\overline{\operatorname{Hom}}_{\mathbb{C}}(T \dot{\Sigma}, E)\right)$, which is asymptotic at $z \in \Gamma^{ \pm}$to $\boldsymbol{A}_{z} \pm \boldsymbol{\delta}_{z}$; denote the latter collection of operators by $\boldsymbol{A}_{\Gamma} \pm \boldsymbol{\delta}_{\Gamma}$. The operator on the weighted space is thus Fredholm if and only if $\mp \boldsymbol{\delta}_{z} \notin \sigma\left(\boldsymbol{A}_{z}\right)$ for all $z \in \Gamma^{ \pm}$, and its index can then be read off again from (2.1), but with $\boldsymbol{A}_{\Gamma} \pm \boldsymbol{\delta}_{\Gamma}$ replacing $\boldsymbol{A}_{\Gamma}$. Note in particular that if all $\boldsymbol{A}_{z}$ are nondegenerate and all $\boldsymbol{\delta}_{z}$ are sufficiently close to 0 , then the weighting does not change the index of $\boldsymbol{D}$.

2.2. The line bundle case. For the rest of this section we assume $n=1$, so each asymptotic operator is equivalent to an unbounded self-adjoint operator on $L^{2}\left(S^{1}, \mathbb{R}^{2}\right)$ of the form $A=-J_{0} \frac{d}{d t}-S(t)$, whose eigenfunctions can be assigned winding numbers. For $\lambda \in \sigma(\boldsymbol{A})$ define $w(\lambda) \in \mathbb{Z}$ to be the winding number of any nontrivial section in the $\lambda$-eigenspace of $\boldsymbol{A}$; this number depends only on $\lambda$, by a result in [HWZ95a]. Moreover, it is shown in the same paper that $w(\lambda)$ is an increasing function of $\lambda$ which takes every integer value exactly twice (counting multiplicity). We define

$$
\begin{aligned}
\alpha_{-}(\boldsymbol{A}) & =\max \{w(\lambda) \mid \lambda \in \sigma(\boldsymbol{A}), \lambda<0\}, \\
\alpha_{+}(\boldsymbol{A}) & =\min \{w(\lambda) \mid \lambda \in \sigma(\boldsymbol{A}), \lambda>0\}, \\
p(\boldsymbol{A}) & =\alpha_{+}(\boldsymbol{A})-\alpha_{-}(\boldsymbol{A}),
\end{aligned}
$$


so if $\boldsymbol{A}$ is nondegenerate, $p(\boldsymbol{A}) \in\{0,1\}$. By another result in [HWZ95a], these winding numbers are related to the Conley-Zehnder index by

$$
\mu_{\mathrm{CZ}}(\boldsymbol{A})=2 \alpha_{-}(\boldsymbol{A})+p(\boldsymbol{A})=2 \alpha_{+}(\boldsymbol{A})-p(\boldsymbol{A}) .
$$

This entire discussion applies also to the operators $\boldsymbol{A}_{z}$ once trivializations $\Phi$ are specified; we thus denote these winding numbers by $\alpha_{ \pm}^{\Phi}\left(\boldsymbol{A}_{z}\right)$, and observe that $p\left(\boldsymbol{A}_{z}\right) \in\{0,1\}$ does not depend on $\Phi$. The latter is the parity of the puncture $z \in \Gamma$, defining a partition of $\Gamma$ into sets of even and odd punctures, denoted $\Gamma_{0}$ and $\Gamma_{1}$ respectively.

Define the $\frac{1}{2} \mathbb{Z}$-valued adjusted first Chern number of $\left(E, \ell, A_{\Gamma}\right)$ by

$$
c_{1}\left(E, \ell, \boldsymbol{A}_{\Gamma}\right)=c_{1}^{\Phi}(E)+\frac{1}{2} \mu^{\Phi}(E, \ell)+\sum_{z \in \Gamma^{+}} \alpha_{-}^{\Phi}\left(\boldsymbol{A}_{z}\right)-\sum_{z \in \Gamma^{-}} \alpha_{+}^{\Phi}\left(\boldsymbol{A}_{z}\right),
$$

and observe that this does not depend on $\Phi$. Using (2.3) and the index formula, it is easy to show that

$$
2 c_{1}\left(E, \ell, \boldsymbol{A}_{\Gamma}\right)=\operatorname{ind}(\boldsymbol{D})-2+2 g+\# \Gamma_{0}+m .
$$

Note that $c_{1}\left(E, \ell, A_{\Gamma}\right)$ is necessarily an integer if $\partial \Sigma=\emptyset$.

The adjusted first Chern number has the following interpretation which justifies its name. If $v \in \operatorname{ker} \boldsymbol{D}$ is a nontrivial section, then the equation $\boldsymbol{D} v=0$ together with the similarity principle implies that $v$ has only isolated zeroes, all of positive order. Moreover, by arguments in [HWZ96], [Sie08], $v$ satisfies an asymptotic formula of the form

$$
v(s, t)=e^{\lambda s}\left(e_{\lambda}(t)+r(s, t)\right)
$$

in admissible trivializations near each puncture $z \in \Gamma^{ \pm}$, where $\lambda \in \sigma\left(\boldsymbol{A}_{z}\right)$ satisfies $\pm \lambda<0, e_{\lambda} \in \Gamma\left(\left.E\right|_{\delta_{z}}\right)$ is a section in the corresponding eigenspace and the remainder $r(s, t)$ goes to zero as $s \rightarrow \pm \infty$. It follows that $v(s, t)$ has only finitely many zeroes, and near $z \in \Gamma^{ \pm}$it has a well defined asymptotic winding number wind ${ }_{z}^{\Phi}(v) \in \mathbb{Z}$, which is bounded from above by $\alpha_{-}^{\Phi}\left(\boldsymbol{A}_{z}\right)$ if $z \in \Gamma^{+}$, or from below by $\alpha_{+}^{\Phi}\left(\boldsymbol{A}_{z}\right)$ if $z \in \Gamma^{-}$. We use this to define the asymptotic vanishing of $v$ :

$$
Z_{\infty}(v)=\sum_{z \in \Gamma^{+}}\left[\alpha_{-}^{\Phi}\left(\boldsymbol{A}_{z}\right)-\operatorname{wind}_{z}^{\Phi}(v)\right]+\sum_{z \in \Gamma^{-}}\left[\operatorname{wind}_{z}^{\Phi}(v)-\alpha_{+}^{\Phi}\left(\boldsymbol{A}_{z}\right)\right]
$$

Define also the $\frac{1}{2} \mathbb{Z}$-valued count of zeroes,

$$
Z(v)=\sum_{z \in v^{-1}(0) \cap \operatorname{int} \dot{\Sigma}} \operatorname{ord}(v ; z)+\frac{1}{2} \sum_{z \in v^{-1}(0) \cap \partial \Sigma} \operatorname{ord}(v ; z),
$$


where the order of a zero on the boundary is defined by a doubling argument described in the appendix. Now a simple computation using these definitions and Proposition A.2 yields the relation

$$
Z(v)+Z_{\infty}(v)=c_{1}\left(E, \ell, \boldsymbol{A}_{\Gamma}\right) .
$$

Observe that both terms on the left hand side are manifestly nonnegative.

The next result is the main objective of this section. Recall from (1.4) the nonnegative integer $K(c, G)$.

Proposition 2.2. (1) In the case ind $(\boldsymbol{D}) \leq 0, \boldsymbol{D}$ is injective if $c_{1}\left(E, \ell, \boldsymbol{A}_{\Gamma}\right)<0$, and otherwise

$$
\operatorname{dim} \operatorname{ker} \boldsymbol{D} \leq K\left(c_{1}\left(E, \ell, \boldsymbol{A}_{\Gamma}\right), \# \Gamma_{0}\right) .
$$

(2) In the case $\operatorname{ind}(\boldsymbol{D}) \geq 0, \boldsymbol{D}$ is surjective if $\operatorname{ind}(\boldsymbol{D})>c_{1}\left(E, \ell, \boldsymbol{A}_{\Gamma}\right)$, and otherwise

$$
\operatorname{ind}(\boldsymbol{D}) \leq \operatorname{dim} \operatorname{ker} \boldsymbol{D} \leq \operatorname{ind}(\boldsymbol{D})+K\left(c_{1}\left(E, \ell, \boldsymbol{A}_{\Gamma}\right)-\operatorname{ind}(\boldsymbol{D}), \# \Gamma_{0}\right) .
$$

Proof. The argument rests crucially on (2.7), together with the observation that if $z \in$ $\Gamma_{0}$, then the space of eigenfunctions with negative eigenvalue and winding $\alpha_{-}^{\Phi}\left(\boldsymbol{A}_{z}\right)$ is 1-dimensional, as is the space with positive eigenvalue and winding $\alpha_{+}^{\Phi}\left(\boldsymbol{A}_{z}\right)$.

We prove the result first for the case $\operatorname{ind}(\boldsymbol{D}) \leq 0$. If $c_{1}\left(E, \ell, \boldsymbol{A}_{\Gamma}\right)<0$, then $\boldsymbol{D}$ is clearly injective, else (2.7) would force any nontrivial section $v \in \operatorname{ker} \boldsymbol{D}$ to have either $Z(v)<0$ or $Z_{\infty}(v)<0$. To establish the dimension bound for ker $\boldsymbol{D}$ when $c_{1}\left(E, \ell, \boldsymbol{A}_{\Gamma}\right) \geq 0$, choose any nonnegative integers $k$ and $n$ such that $k \leq$ $\# \Gamma_{0}$ and $2 k+n>2 c_{1}\left(E, \ell, \boldsymbol{A}_{\Gamma}\right)$. In this situation we can construct an injective homomorphism from ker $\boldsymbol{D}$ into a real vector space of dimension $n+k$. Indeed, if $\partial \Sigma \neq \emptyset$, pick $n$ distinct points $\zeta_{1}, \ldots, \zeta_{n} \in \partial \Sigma$, and choose also $k$ distinct even punctures $z_{1}, \ldots, z_{k} \in \Gamma_{0}$. For each of the $z_{j}$, the correspondence $v \mapsto e_{\lambda}$ coming from the asymptotic formula (2.6) defines a linear map from $\operatorname{ker} \boldsymbol{D}$ into the 1-dimensional vector space $V_{z_{j}}$ consisting of eigenfunctions of $\boldsymbol{A}_{z_{j}}$ with winding equal to $\alpha_{\mp}^{\Phi}\left(\boldsymbol{A}_{z_{j}}\right)$. We can define this map so that it takes the value $0 \in V_{z_{j}}$ if and only if the eigenfunction in (2.6) has a different winding number. Using these maps and the evaluation of $v$ at the points $\zeta_{j} \in \partial \Sigma$, we obtain a homomorphism

$$
\Psi: \operatorname{ker} \boldsymbol{D} \rightarrow \ell_{\zeta_{1}} \oplus \cdots \oplus \ell_{\zeta_{n}} \oplus V_{z_{1}} \oplus \cdots \oplus V_{z_{k}} .
$$

The claim is that $\Psi$ is injective, and thus $\operatorname{dim} \operatorname{ker} \boldsymbol{D} \leq n+k$. Indeed, suppose $v \in \operatorname{ker} \boldsymbol{D}$ is a nontrivial section with $\Psi(v)=0$. Then the asymptotic winding of $v$ differs from $\alpha_{+}^{\Phi}\left(\boldsymbol{A}_{z_{j}}\right)$ at each of the punctures $z_{j}$, implying $Z_{\infty}(v) \geq k$. Similarly, $v$ has boundary zeroes at $\zeta_{1}, \ldots, \zeta_{n}$, contributing at least $n / 2$ to $Z(v)$, hence

$$
c_{1}\left(E, \ell, \boldsymbol{A}_{\Gamma}\right)=Z(v)+Z_{\infty}(v) \geq \frac{n}{2}+k,
$$


which contradicts our assumptions on $n$ and $k$.

A minor modification to this argument is needed if $\partial \Sigma=\emptyset$. We must now assume $n$ is even, and choose distinct interior points $\zeta_{1}, \ldots, \zeta_{n / 2} \in \dot{\Sigma}$, using evaluation at these points to define the homomorphism

$$
\Psi: \operatorname{ker} \boldsymbol{D} \rightarrow E_{\zeta_{1}} \oplus \cdots \oplus E_{\zeta_{n / 2}} \oplus V_{z_{1}} \oplus \cdots \oplus V_{z_{k}} .
$$

The right hand side is again a vector space of real dimension $n+k$, and the same argument as above shows that $\Psi$ is injective.

To deal with the case ind $(\boldsymbol{D}) \geq 0$, we consider the formal adjoint $\boldsymbol{D}^{*}$ (cf. [Sch95]). This can be regarded as a Cauchy-Riemann type operator on the bundle $F:=$ $\overline{\operatorname{Hom}}_{\mathbb{C}}(T \dot{\Sigma}, E) \rightarrow \dot{\Sigma}$ with an appropriate totally real boundary condition $\ell^{*}$ and asymptotic operators $\boldsymbol{A}_{z}^{*}$, which have the same parity as $\boldsymbol{A}_{z}$. It satisfies

$$
\operatorname{ind}\left(\boldsymbol{D}^{*}\right)=-\operatorname{ind}(\boldsymbol{D}), \quad \operatorname{dim} \operatorname{ker} \boldsymbol{D}^{*}=\operatorname{dim} \operatorname{coker} \boldsymbol{D},
$$

and applying (2.5) to $\boldsymbol{D}$ and $\boldsymbol{D}^{*}$ together, we find

$$
c_{1}\left(E, \ell, \boldsymbol{A}_{\Gamma}\right)-c_{1}\left(F, \ell^{*}, \boldsymbol{A}_{\Gamma}^{*}\right)=\frac{1}{2}\left[\operatorname{ind}(\boldsymbol{D})-\operatorname{ind}\left(\boldsymbol{D}^{*}\right)\right]=\operatorname{ind}(\boldsymbol{D}) .
$$

Then the condition $c_{1}\left(F, \ell^{*}, \boldsymbol{A}_{\Gamma}^{*}\right)<0$ is satisfied if and only if ind $(\boldsymbol{D})>c_{1}\left(E, \ell, \boldsymbol{A}_{\Gamma}\right)$, and this implies $\boldsymbol{D}$ is surjective. If ind $(\boldsymbol{D}) \leq c_{1}\left(E, \ell, \boldsymbol{A}_{\Gamma}\right)$, then $c_{1}\left(F, \ell^{*}, \boldsymbol{A}_{\Gamma}^{*}\right) \geq 0$ and we can apply the above estimate to $\operatorname{dim} \operatorname{ker} \boldsymbol{D}^{*}$, giving

$$
\begin{aligned}
\operatorname{dim} \operatorname{ker} \boldsymbol{D} & =\operatorname{ind}(\boldsymbol{D})+\operatorname{dim} \operatorname{coker} \boldsymbol{D}=\operatorname{ind}(\boldsymbol{D})+\operatorname{dim} \operatorname{ker} \boldsymbol{D}^{*} \\
& \leq \operatorname{ind}(\boldsymbol{D})+K\left(c_{1}\left(F, \ell^{*}, \boldsymbol{A}_{\Gamma}^{*}\right), \# \Gamma_{0}\right) \\
& =\operatorname{ind}(\boldsymbol{D})+K\left(c_{1}\left(E, \ell, \boldsymbol{A}_{\Gamma}\right)-\operatorname{ind}(\boldsymbol{D}), \# \Gamma_{0}\right) .
\end{aligned}
$$

Remark 2.3. The proof of Theorem 2 requires only the very simplest case of this dimension bound, namely that $\operatorname{ker} \boldsymbol{D}$ is trivial when $c_{1}\left(E, \ell, \boldsymbol{A}_{\Gamma}\right)<0$. As that proof will demonstrate, however, such bounds can sometimes be useful in cases where $\boldsymbol{D}$ is not surjective, so perhaps the more general dimension bound will eventually find similar application.

\section{The normal operator for a holomorphic curve}

In this section we will give the precise definition of regularity and show that it is equivalent to the surjectivity of a certain Cauchy-Riemann operator on a generalized normal bundle. The precise relation between this operator and the concept of regularity is stated in $\$ 3.4$ as Theorem 3, and in $\S 3.5$ we apply the linear transversality theory from $\$ 2.2$ to show that Theorem 1 follows as an easy corollary. 
Throughout the following, we fix a compact, connected and oriented surface $\Sigma$ of genus $g \geq 0$ with $m \geq 0$ boundary components, and a finite set $\Gamma \subset$ int $\Sigma$, writing $\dot{\Sigma}=\Sigma \backslash \Gamma$.

3.1. Teichmüller slices and Cauchy-Riemann operators. We begin by collecting some classical facts about moduli spaces of Riemann surfaces which can be related to the analysis of Cauchy-Riemann type operators.

Let $g(\Sigma)$ denote the space of smooth complex structures on $\Sigma$ that induce the given orientation, and denote by Diff $+(\Sigma, \Gamma)$ the group of orientation preserving diffeomorphisms on $\Sigma$ that fix $\Gamma$, and $\operatorname{Diff}_{0}(\Sigma, \Gamma) \subset \operatorname{Diff}_{+}(\Sigma, \Gamma)$ those which are homotopic to the identity. Both of these groups act on $\mathcal{g}(\Sigma)$ by $(\varphi, j) \mapsto \varphi^{*} j$, and the Teichmüller space of $\dot{\Sigma}$ is a smooth finite-dimensional manifold defined as

$$
\mathcal{T}(\dot{\Sigma})=\mathscr{g}(\Sigma) / \operatorname{Diff}_{0}(\Sigma, \Gamma) .
$$

Its quotient by the mapping class group $M(\dot{\Sigma})=\operatorname{Diff}_{+}(\Sigma, \Gamma) / \operatorname{Diff}_{0}(\Sigma, \Gamma)$ gives the moduli space of Riemann surfaces (with genus $g, m$ boundary components and $\# \Gamma$ interior marked points)

$$
\mathcal{M}(\dot{\Sigma})=\mathcal{T}(\dot{\Sigma}) / M(\dot{\Sigma})=\mathcal{G}(\Sigma) / \operatorname{Diff}_{+}(\Sigma, \Gamma),
$$

which is in general an orbifold of the same dimension. We say $\dot{\Sigma}$ is stable if $\chi(\dot{\Sigma})<0$, in which case

$$
\operatorname{dim} \mathcal{M}(\dot{\Sigma})=6 g-6+3 m+2 \# \Gamma=-3 \chi(\dot{\Sigma})-\# \Gamma,
$$

and the automorphism group

$$
\operatorname{Aut}(\dot{\Sigma}, j)=\left\{\varphi \in \operatorname{Diff}_{+}(\Sigma, \Gamma) \mid \varphi^{*} j=j\right\}
$$

is finite for any choice of $j \in \mathcal{G}(\Sigma)$ (though its order may depend on $j$ ). Let $\mathbb{D} \subset \mathbb{C}$ denote the closed unit disk, $\mathbb{A}=[0,1] \times S^{1}$ the compact annulus and $\mathbb{T}^{2}=\mathbb{R}^{2} / \mathbb{Z}^{2}$ the 2-dimensional torus. For our purposes, the non-stable cases to be considered are the following:

(1) $\mathcal{M}\left(S^{2}\right)=\{[i]\}$ and $\operatorname{dim} \operatorname{Aut}\left(S^{2}, i\right)=6$.

(2) $\mathcal{M}(\mathbb{C})=\{[i]\}$ and $\operatorname{dim} \operatorname{Aut}(\mathbb{C}, i)=4$.

(3) $\mathcal{M}(\mathbb{D})=\{[i]\}$ and $\operatorname{dim} \operatorname{Aut}(\mathbb{D}, i)=3$.

(4) $\mathcal{M}\left(\mathbb{R} \times S^{1}\right)=\{[i]\}$ and $\operatorname{dim} \operatorname{Aut}\left(\mathbb{R} \times S^{1}, i\right)=2$.

(5) $\mathcal{M}(\mathbb{D} \backslash\{0\})=\{[i]\}$ and $\operatorname{dim} \operatorname{Aut}(\mathbb{D} \backslash\{0\}, i)=1$.

(6) $\operatorname{dim} \mathcal{M}(\mathbb{A})=1$ and $\operatorname{dim} \operatorname{Aut}(\mathbb{A}, j)=1$.

(7) $\operatorname{dim} \mathcal{M}\left(\mathbb{T}^{2}\right)=2$ and $\operatorname{dim} \operatorname{Aut}\left(\mathbb{T}^{2}, j\right)=2$. 
For all but the last case, the mapping class group $M(\dot{\Sigma})$ is trivial and thus $\mathcal{M}(\dot{\Sigma})=$ $\mathcal{T}(\dot{\Sigma})$ is a manifold. Observe also that if $\dot{\Sigma}$ is not stable,

$$
\operatorname{dim} \operatorname{Aut}(\dot{\Sigma}, j)-\operatorname{dim} \mathcal{M}(\dot{\Sigma})=3 \chi(\dot{\Sigma})+\# \Gamma .
$$

Fixing $p>2$, the latter is the Fredholm index of the standard linear Cauchy-Riemann operator

$$
\boldsymbol{D}_{\Gamma}^{\Sigma}: W_{T(\partial \Sigma)}^{1, p}(T \Sigma ; \Gamma) \rightarrow L^{p}\left(\overline{\operatorname{End}}_{\mathbb{C}}(T \Sigma)\right),
$$

where $W_{T(\partial \Sigma)}^{1, p}(T \Sigma ; \Gamma)$ is the space of $W^{1, p}$-smooth vector fields $Y: \Sigma \rightarrow T \Sigma$ satisfying $Y(\partial \Sigma) \subset T(\partial \Sigma)$ and $\left.Y\right|_{\Gamma}=0$.

Lemma 3.1. For all choices of $(\Sigma, j, \Gamma), \operatorname{dim} \operatorname{coker}\left(\boldsymbol{D}_{\Gamma}^{\Sigma}\right)=\operatorname{dim} \mathcal{M}(\dot{\Sigma})$.

Proof. This may be regarded as a standard piece of Teichmüller theory in the stable case (cf. [Tro92]), and also follows by using a simplified version of the argument in the proof of Proposition 2.2 to show that $\boldsymbol{D}_{\Gamma}^{\Sigma}$ is injective. Here one must account also for the condition $\left.Y\right|_{\Gamma}=0$, which ensures $Z(Y) \geq \# \Gamma$, thus it suffices to observe that the adjusted first Chern number is strictly less than \# $\Gamma$. In the non-stable case, a similar argument shows that $\operatorname{dim} \operatorname{ker}\left(\boldsymbol{D}_{\Gamma}^{\Sigma}\right) \leq \operatorname{dim} \operatorname{Aut}(\dot{\Sigma}, j)$, and by interpreting $\boldsymbol{D}_{\Gamma}^{\Sigma}$ as the linearization of a nonlinear operator $\bar{\partial}_{j} \varphi=T \varphi+j \circ T \varphi \circ j$, one sees that $\operatorname{ker}\left(\boldsymbol{D}_{\Gamma}^{\Sigma}\right)$ contains aut $(\dot{\Sigma}, j)$, giving an inequality in the other direction, hence

$$
\operatorname{dim} \operatorname{ker}\left(\boldsymbol{D}_{\Gamma}^{\Sigma}\right)=\operatorname{dim} \operatorname{Aut}(\dot{\Sigma}, j)
$$

The result then follows from (3.1).

Given $j \in \mathcal{L}(\Sigma)$ and the corresponding Cauchy-Riemann operator $\boldsymbol{D}_{\Gamma}^{\Sigma}$, pick a complement of $\operatorname{im}\left(D_{\Gamma}^{\Sigma}\right)$, i.e. a subspace $C \subset L^{p}\left(\overline{\operatorname{End}}_{\mathbb{C}}(T \Sigma)\right)$ such that

$$
\operatorname{im}\left(D_{\Gamma}^{\Sigma}\right) \oplus C=L^{p}\left(\overline{\operatorname{End}}_{\mathbb{C}}(T \Sigma)\right) .
$$

By approximation, we may assume every section in $C$ is smooth and vanishes on a neighborhood of $\Gamma$. We can then choose a small neighborhood $\mathcal{O} \subset C$ of 0 and define the map

$$
\Phi: \mathcal{O} \rightarrow \mathcal{g}(\Sigma), \quad y \mapsto\left(\mathbb{1}+\frac{1}{2} j y\right) j\left(\mathbb{1}+\frac{1}{2} j y\right)^{-1},
$$

which has the properties $\Phi(0)=j$ and

$$
\left.\frac{\partial}{\partial t} \Phi(t y)\right|_{t=0}=y
$$


thus it is injective if $\mathcal{O}$ is sufficiently small. The image

$$
\mathcal{T}:=\Phi(\mathcal{O}) \subset \mathcal{J}(\Sigma)
$$

is thus a smooth manifold of dimension $\operatorname{dim} C=\operatorname{dim} \mathcal{T}(\dot{\Sigma})$, with $T_{j} \mathcal{T}=C$ and consisting of smooth complex structures close to $j$ that are identical to $j$ on some fixed neighborhood of $\Gamma$. It parametrizes a neighborhood of $[j]$ in $\mathcal{T}(\dot{\Sigma})$, i.e. the projection $\mathcal{g}(\Sigma) \rightarrow \mathcal{T}(\dot{\Sigma})$ restricts to a diffeomorphism from $\mathcal{T}$ onto a neighborhood of $[j]$. This provides an explicit construction of the following general object:

Definition 3.2. Given $j \in \mathcal{L}(\Sigma)$, we define a Teichmüller slice through $j$ to be any smooth family $\mathcal{T} \subset \mathcal{L}(\Sigma)$ parametrized by an injective map $U \rightarrow \mathcal{L}(\Sigma)$, where $\mathcal{U}$ is an open subset of $\mathbb{R}^{\operatorname{dim} \mathcal{T}(\dot{\Sigma})}$, such that all $j^{\prime} \in \mathcal{T}$ are identical on some fixed neighborhood of $\Gamma$, and $\operatorname{im}\left(\boldsymbol{D}_{\Gamma}^{\Sigma}\right) \oplus T_{j} \mathcal{T}=L^{p}\left(\overline{\operatorname{End}}_{\mathbb{C}}(T \Sigma)\right)$.

Lemma 3.3. If $(\dot{\Sigma}, j)$ is stable, then there exists a Teichmüller slice $\mathcal{T}$ through $j$ that is invariant under the group action $\operatorname{Aut}(\dot{\Sigma}, j) \times \mathcal{L}(\Sigma) \rightarrow \mathcal{J}(\Sigma),\left(\varphi, j^{\prime}\right) \mapsto \varphi^{*} j^{\prime}$.

Proof. The automorphism group $G:=\operatorname{Aut}(\dot{\Sigma})$ is finite and consists of biholomorphic maps on $(\Sigma, j)$ that fix $\Gamma$. Each point in $\Gamma$ then has a $G$-invariant neighborhood biholomorphically equivalent to the standard unit disk, on which $G$ acts by rational rotations. Let $g$ denote a metric on $\Sigma$ that is invariant under the action of $G$; such a metric can be constructed by starting from the Poincaré metric on $\dot{\Sigma}$ and interpolating this with flat rotation-invariant metrics on disk-like neighborhoods of each point in $\Gamma$. Then $g$ induces a bundle metric on $\overline{\operatorname{End}}_{\mathbb{C}}(T \Sigma) \rightarrow \Sigma$ and a corresponding $G$-invariant $L^{2}$-inner product $\langle,\rangle_{L^{2}}$ on the space of sections of this bundle.

To prove the lemma, it suffices to construct a $G$-invariant complement $C \subset$ $L^{p}\left(\overline{\operatorname{End}}_{\mathbb{C}}(T \Sigma)\right)$ of $\operatorname{im}\left(\boldsymbol{D}_{\Gamma}^{\Sigma}\right)$ that consists of smooth sections vanishing near $\Gamma$ : then an appropriate Teichmüller slice can be defined via (3.2) since $\varphi^{*} j=j$ implies $\Phi\left(\varphi^{*} y\right)=\varphi^{*} \Phi(y)$ for any $\varphi \in G$. Observe that $\operatorname{im}\left(\boldsymbol{D}_{\Gamma}^{\Sigma}\right)$ itself is $G$-invariant, since $\varphi^{*} j=j$ also implies $\boldsymbol{D}_{\Gamma}^{\Sigma}\left(\varphi^{*} Y\right)=\varphi^{*}\left(\boldsymbol{D}_{\Gamma}^{\Sigma} Y\right)$ for all $Y \in W_{T(\partial \Sigma)}^{1, p}(T \Sigma ; \Gamma)$. Now using the $G$-invariant $L^{2}$-product chosen above, define a complement $C_{0}$ as the $L^{2}$-orthogonal complement of $\operatorname{im}\left(\boldsymbol{D}_{\Gamma}^{\Sigma}\right)$, i.e.

$$
C_{0}=\left\{y \in L^{p}\left(\overline{\operatorname{End}}_{\mathbb{C}}(T \Sigma)\right) \mid\left\langle\boldsymbol{D}_{\Gamma}^{\Sigma} Y, y\right\rangle_{L^{2}}=0 \text { for all } Y \in W_{T(\partial \Sigma)}^{1, p}(T \Sigma ; \Gamma)\right\} .
$$

This space is $G$-invariant due to the $G$-invariance of $\operatorname{im}\left(D_{\Gamma}^{\Sigma}\right)$ and $\langle,\rangle_{L^{2}}$, and by elliptic regularity for weak solutions of the formal adjoint equation, it consists only of smooth sections. Now choosing $G$-invariant disk-like neighborhoods of the points in $\Gamma$, we can obtain the desired complement $C$ by multiplying the sections in $C_{0}$ by $G$-invariant cutoff functions that vanish near $G$ and equal 1 outside a sufficiently small neighborhood of $\Gamma$. 
For the two non-stable cases in which $\mathcal{T}(\dot{\Sigma})$ is nontrivial, it will be convenient to have explicit global Teichmüller slices. If $\dot{\Sigma}=\mathbb{A}=[0,1] \times S^{1}$, for each $\tau>0$ define the diffeomorphism $\varphi_{\tau}: \mathbb{A} \rightarrow[0, \tau] \times S^{1},(s, t) \mapsto(\tau s, t)$ and let $\mathcal{T}_{\mathbb{A}}$ denote the collection of complex structures $\left\{\varphi_{\tau}^{*} i\right\}_{\tau>0}$. This parametrizes the entirety of $\mathcal{T}(\mathbb{A})$ (which equals $\mathcal{M}(\mathbb{A})$ since the mapping class group is trivial), and also gives a natural identification of every $\operatorname{Aut}\left(\mathbb{A}, \varphi_{\tau}^{*} i\right)$ with $S^{1}$, acting on $\mathbb{A}$ by translation of the second factor. If $\dot{\Sigma}=\mathbb{T}^{2}=\mathbb{R}^{2} / \mathbb{Z}^{2}$, we define $\mathcal{T}_{\mathbb{T}^{2}}$ to be the space of all constant complex structures on $\mathbb{R}^{2}=\mathbb{C}$ that are compatible with the standard orientation; clearly these descend to $\mathbb{T}^{2}$, and they also parametrize the entirety of $\mathcal{T}\left(\mathbb{T}^{2}\right)$. Then for each $j \in \mathcal{T}_{\mathbb{T}^{2}}$, the subgroup

$$
\operatorname{Aut}_{0}\left(\mathbb{T}^{2}, j\right):=\operatorname{Aut}\left(\mathbb{T}^{2}, j\right) \cap \operatorname{Diff}_{0}\left(\mathbb{T}^{2}\right)
$$

can be identified naturally with $\mathbb{T}^{2}$, acting by translations. Choosing a base point $p=[(0,0)] \in \mathbb{T}^{2}$, the stabilizer of $[j] \in \mathcal{T}\left(\mathbb{T}^{2}\right)$ under the action of $M\left(\mathbb{T}^{2}\right)=$ $\operatorname{SL}(2, \mathbb{Z})$ is meanwhile the finite subgroup

$$
\operatorname{Aut}\left(\mathbb{T}^{2}, j ; p\right):=\left\{\varphi \in \operatorname{Aut}\left(\mathbb{T}^{2}, j\right) \mid \varphi(p)=p\right\}=\{A \in \operatorname{SL}(2, \mathbb{Z}) \mid A j=j A\},
$$

and Aut $\left(\mathbb{T}^{2}, j\right)$ is the semidirect product of Aut $\left(\mathbb{T}^{2}, j ; p\right)$ with $\operatorname{Aut}_{0}\left(\mathbb{T}^{2}, j\right)=\mathbb{T}^{2}$. Note in particular that for any $j \in \mathcal{T}_{\mathbb{T}^{2}}$, this group acts by affine transformations on $\mathbb{R}^{2}$ (descending to $\mathbb{T}^{2}$ ), and the action $\left(\varphi, j^{\prime}\right) \mapsto \varphi^{*} j^{\prime}$ therefore preserves $\mathcal{T}_{\mathbb{T}^{2}}$.

The following will be useful for technical reasons in our analysis of the relationship between $\boldsymbol{D}_{u}$ and its normal component.

Lemma 3.4. For any $j \in \mathcal{G}(\Sigma)$ and finite set $K \subset \dot{\Sigma}$, there exists a Teichmüller slice $\mathcal{T}$ through $j$ such that every $j^{\prime} \in \mathcal{T}$ is identical to $j$ on some fixed neighborhood of $K \cup \Gamma$.

Proof. It suffices to construct $C=T_{j} \mathcal{T}$ so that every $y \in C$ vanishes near $K \cup \Gamma$. This can be done using cutoff functions to replace a basis of any given complement with one that vanishes in such a neighborhood; the new basis can be made $L^{p}$-close to the old one if the neighborhood is sufficiently small.

For any Teichmüller slice $\mathcal{T}$ through $j$, the operator

$$
\boldsymbol{L}_{\Gamma}: T_{j} \mathcal{T} \oplus W^{1, p}(T \Sigma ; \Gamma) \rightarrow L^{p}\left(\overline{\operatorname{End}}_{\mathbb{C}}(T \Sigma)\right), \quad(y, Y) \mapsto j y+\boldsymbol{D}_{\Gamma}^{\Sigma} Y,
$$

is clearly surjective; indeed, as $\boldsymbol{D}_{\Gamma}^{\Sigma}$ is complex linear, $\boldsymbol{L}_{\Gamma}(y, j Y)=j\left(y+\boldsymbol{D}_{\Gamma}^{\Sigma} Y\right)$, and the target space is spanned by $T_{j} \mathcal{T}$ and $\operatorname{im}\left(\boldsymbol{D}_{\Gamma}^{\Sigma}\right)$. For the analysis in the following sections it will be useful to derive a corresponding statement for the standard CauchyRiemann operator on a Riemann surface with ends. We will recall in the next section the construction of certain Banach manifolds containing asymptotically cylindrical 
maps $\dot{\Sigma} \rightarrow W$. In the simple case $W=\dot{\Sigma}$, the tangent space to such a Banach manifold $\mathscr{B}^{\Sigma}$ at the identity map $\mathbb{1}: \dot{\Sigma} \rightarrow \dot{\Sigma}$ can be written as

$$
T_{\mathbb{1}} \mathcal{B}^{\Sigma}=W_{T(\partial \Sigma)}^{1, p, \delta}(T \dot{\Sigma}) \oplus V_{\Gamma}^{\Sigma},
$$

where $\delta>0$ is a small weight applying at every end and $V_{\Gamma}^{\Sigma} \subset \Gamma(T \dot{\Sigma})$ is a $2 \# \Gamma$ dimensional space of smooth sections that are supported near infinity and constant in some fixed choice of cylindrical coordinates near each end. The natural nonlinear Cauchy-Riemann operator defines a section of a Banach space bundle over $\mathscr{B}^{\Sigma}$, whose linearization at $\mathbb{1}$ is the usual linear Cauchy-Riemann operator given by the holomorphic structure of $T \dot{\Sigma} \rightarrow \dot{\Sigma}$, denoted here by

$$
D^{\Sigma}: W_{T(\partial \Sigma)}^{1, p, \boldsymbol{\delta}}(T \dot{\Sigma}) \oplus V_{\Gamma}^{\Sigma} \rightarrow L^{p, \boldsymbol{\delta}}\left(\overline{\operatorname{End}}_{\mathbb{C}}(T \dot{\Sigma})\right) .
$$

Now since every $y \in T_{j} \mathcal{T}$ is smooth and vanishes near $\Gamma$, there is a natural inclusion of $T_{j} \mathcal{T} \subset L^{p, \delta}\left(\overline{\operatorname{End}}_{\mathbb{C}}(T \dot{\Sigma})\right)$, as well as a bounded linear map $T_{j} \mathcal{T} \rightarrow$ $L^{p, \delta}\left(\overline{\operatorname{End}}_{\mathbb{C}}(T \dot{\Sigma})\right), y \mapsto j y$.

Lemma 3.5. The operator

$$
\boldsymbol{L}: T_{j} \mathcal{T} \oplus\left(W_{T(\partial \Sigma)}^{1, p, \boldsymbol{\delta}}(T \dot{\Sigma}) \oplus V_{\Gamma}^{\Sigma}\right) \rightarrow L^{p, \boldsymbol{\delta}}\left(\overline{\operatorname{End}}_{\mathbb{C}}(T \dot{\Sigma})\right), \quad(y, \eta) \mapsto j y+D^{\Sigma} \eta,
$$

is surjective.

Proof. Applying the linear theory in $\S 2$, we find that ind $\left(\boldsymbol{D}^{\Sigma}\right)=\operatorname{ind}\left(\boldsymbol{D}_{\Gamma}^{\Sigma}\right)$ and hence $\operatorname{ind}(\boldsymbol{L})=\operatorname{ind}\left(\boldsymbol{L}_{\Gamma}\right)$. Now in light of the natural inclusion

$$
W_{T(\partial \Sigma)}^{1, p, \delta}(T \dot{\Sigma}) \oplus V_{\Gamma}^{\Sigma} \hookrightarrow W_{T(\partial \Sigma)}^{1, p}(T \Sigma ; \Gamma)
$$

it follows that $\operatorname{ker}(\boldsymbol{L}) \subset \operatorname{ker}\left(\boldsymbol{L}_{\Gamma}\right)$, but since $\operatorname{dim} \operatorname{ker}(\boldsymbol{L}) \geq \operatorname{ind}(\boldsymbol{L})$ in general and $\operatorname{dim} \operatorname{ker}\left(\boldsymbol{L}_{\Gamma}\right)=\operatorname{ind}\left(\boldsymbol{L}_{\Gamma}\right)$ by the remarks above, we have

$$
\operatorname{dim} \operatorname{ker}(\boldsymbol{L}) \leq \operatorname{dim} \operatorname{ker}\left(\boldsymbol{L}_{\Gamma}\right)=\operatorname{ind}\left(\boldsymbol{L}_{\Gamma}\right)=\operatorname{ind}(\boldsymbol{L}),
$$

implying $\boldsymbol{L}$ is surjective.

Corollary 3.6. For any Teichmüller slice $\mathcal{T}$ through $j$,

$$
L^{p, \boldsymbol{\delta}}\left(\overline{\operatorname{End}}_{\mathbb{C}}(T \dot{\Sigma})\right)=\operatorname{im}\left(\boldsymbol{D}^{\Sigma}\right) \oplus T_{j} \mathcal{T} .
$$

3.2. Functional analytic setup. To state the definition of regularity, we begin by reviewing the nonlinear functional analytic setup used in [Dra04], [Bou02] for asymptotically cylindrical maps $u: \dot{\Sigma} \rightarrow W$ with nondegenerate or Morse-Bott asymptotic 
orbits. Fix the surface $\Sigma$, punctures $\Gamma=\Gamma^{+} \cup \Gamma^{-} \subset$ int $\Sigma$, and asymptotic constraints c. Recall that the latter choice partitions $\Gamma$ into a set of constrained and unconstrained punctures $\Gamma=\Gamma_{C} \cup \Gamma_{U}$, and assigns to each $z \in \Gamma_{C}^{ \pm}$an orbit $P_{z}$ of $X_{ \pm}$, which we will assume is Morse-Bott, and we will denote its period by $T_{z}$. For each $z \in \Gamma_{U}^{ \pm}$, we instead choose an arbitrary Morse-Bott manifold of periodic orbits in $M_{ \pm}$, denoted again by $P_{z}$, with period $T_{z}>0$. By a slight abuse of notation, each $P_{z}$ may be regarded both as a submanifold $P_{z} \subset M_{ \pm}$and as a set of $T_{z}$-periodic orbits $\gamma \in P_{z}$ (sometimes with only one element). Denote this collection of choices for all punctures $z \in \Gamma$ by $P_{\Gamma}$. We shall then consider a Banach manifold consisting of asymptotically cylindrical maps $u: \dot{\Sigma} \rightarrow W$ whose asymptotic orbits $\gamma_{z}$ for $z \in \Gamma$ satisfy $\gamma_{z} \in P_{z}$.

Before explaining the Banach manifold, we digress for a moment to define some important invariants that enter into the index formula. Recall that any $T$-periodic orbit $\gamma$ of $X_{ \pm}$has an associated asymptotic operator $\boldsymbol{A}_{\gamma}$, defined on sections of the bundle $\xi_{ \pm}$along $\gamma$. One can write it down by choosing a parametrization $x: S^{1} \rightarrow M_{ \pm}$of $\gamma$ with $\lambda(\dot{x}) \equiv T$, and defining $\boldsymbol{A}_{\gamma}: \Gamma\left(x^{*} \xi_{ \pm}\right) \rightarrow \Gamma\left(x^{*} \xi_{ \pm}\right)$by

$$
\boldsymbol{A}_{\gamma} v=-J_{ \pm}\left(\nabla_{t} v-T \nabla X_{ \pm}\right)
$$

for any symmetric connection $\nabla$ on $M_{ \pm}$. This gives an unbounded self-adjoint operator on $L^{2}\left(x^{*} \xi_{ \pm}\right)$of the form considered in $\S 2$, and it is nondegenerate if and only if the orbit is nondegenerate, in which case we define the Conley-Zehnder index $\mu_{\mathrm{CZ}}^{\Phi}(\gamma)=\mu_{\mathrm{CZ}}^{\Phi}\left(\boldsymbol{A}_{\gamma}\right)$ for any choice of trivialization $\Phi$ on $x^{*} \xi_{ \pm}$. If $\gamma$ is degenerate, then $\boldsymbol{A}_{\gamma}$ can be perturbed to a nondegenerate asymptotic operator by adding any number $\boldsymbol{\epsilon} \in \mathbb{R} \backslash-\sigma(\boldsymbol{A})$, and we thus define the perturbed Conley-Zehnder index

$$
\mu_{\mathrm{CZ}}^{\Phi}(\gamma+\boldsymbol{\epsilon}):=\mu_{\mathrm{CZ}}^{\Phi}\left(\boldsymbol{A}_{\gamma}+\boldsymbol{\epsilon}\right),
$$

and its parity

$$
p(\gamma+\boldsymbol{\epsilon})= \begin{cases}0 & \text { if } \mu_{\mathrm{CZ}}^{\Phi}(\gamma+\boldsymbol{\epsilon}) \text { is even, } \\ 1 & \text { if } \mu_{\mathrm{CZ}}^{\Phi}(\gamma+\boldsymbol{\epsilon}) \text { is odd }\end{cases}
$$

which does not depend on $\Phi$. Observe that if $\gamma$ is nondegenerate and $\epsilon$ is sufficiently close to zero, then $\mu_{\mathrm{CZ}}^{\Phi}(\gamma+\boldsymbol{\epsilon})=\mu_{\mathrm{CZ}}^{\Phi}(\gamma)$ since $\sigma\left(\boldsymbol{A}_{\gamma}\right)$ is discrete. More generally, one can see from the relationship between the Conley-Zehnder index and spectral flow (cf. [RS95]) that for sufficiently small $\epsilon>0$,

$$
\mu_{\mathrm{CZ}}^{\Phi}(\gamma-\boldsymbol{\epsilon})-\mu_{\mathrm{CZ}}^{\Phi}(\gamma+\boldsymbol{\epsilon})=\operatorname{dim} \operatorname{ker}\left(\boldsymbol{A}_{\gamma}\right) .
$$

In particular if $\gamma$ belongs to a Morse-Bott family $P$, then the right hand side of (3.3) is $\operatorname{dim} P-1$, and $\mu_{\mathrm{CZ}}^{\Phi}(\gamma \pm \boldsymbol{\epsilon})$ remains unchanged if we move $\gamma$ to a different orbit in the same family. 
If $M_{ \pm}$are 3-dimensional, then $\xi_{ \pm}$have complex rank one, so recalling the definitions in $\$ 2.2$, we can associate to any $T$-periodic orbit $\gamma$ of $X_{ \pm}$and real number $\epsilon$ the so-called extremal winding numbers

$$
\alpha_{ \pm}^{\Phi}(\gamma+\boldsymbol{\epsilon}):=\alpha_{ \pm}^{\Phi}\left(\boldsymbol{A}_{\gamma}+\boldsymbol{\epsilon}\right)
$$

or for the case $\boldsymbol{\epsilon}=0$, simply $\alpha_{ \pm}^{\Phi}(\gamma)=\alpha_{ \pm}^{\Phi}\left(\boldsymbol{A}_{\gamma}\right)$. We will refer to the eigenfunctions of $\boldsymbol{A}_{\gamma}$ involved in this definition as extremal eigenfunctions at $\gamma$ if $\boldsymbol{\epsilon}=0$, or more generally extremal eigenfunctions with respect to $\boldsymbol{\epsilon}$. Now if $\boldsymbol{\epsilon} \notin-\sigma\left(\boldsymbol{A}_{\gamma}\right)$, (2.3) gives

$$
\begin{aligned}
\mu_{\mathrm{CZ}}^{\Phi}(\gamma+\boldsymbol{\epsilon}) & =2 \alpha_{\mp}^{\Phi}(\gamma+\boldsymbol{\epsilon}) \pm p(\gamma+\boldsymbol{\epsilon}), \\
p(\gamma+\boldsymbol{\epsilon}) & =\alpha_{+}^{\Phi}(\gamma+\boldsymbol{\epsilon})-\alpha_{-}^{\Phi}(\gamma+\boldsymbol{\epsilon}) \in\{0,1\} .
\end{aligned}
$$

Choosing $\delta>0$ arbitrarily small, it will also be convenient to define

$$
v_{ \pm}(\gamma)=\alpha_{ \pm}^{\Phi}(\gamma-\delta)-\alpha_{ \pm}^{\Phi}(\gamma+\delta),
$$

which equals 0 whenever $\gamma$ is nondegenerate, and is otherwise either 0 or $1 .^{6}$

Notation. Fix a number $\boldsymbol{\delta}>0$, which we will generally assume to be as small as may be needed. Suppose $\Gamma=\Gamma^{+} \cup \Gamma^{-}$is a set of punctures and $\mathbf{c}$ is a set of asymptotic constraints, defining constrained and unconstrained subsets $\Gamma_{C}, \Gamma_{U} \subset \Gamma$ respectively. We then associate to each puncture $z \in \Gamma$ a real number

$$
\mathbf{c}_{z}:= \begin{cases}\boldsymbol{\delta} & \text { if } z \in \Gamma_{C}, \\ -\boldsymbol{\delta} & \text { if } z \in \Gamma_{U} .\end{cases}
$$

For asymptotically cylindrical maps $u: \dot{\Sigma} \rightarrow W$ subject to constraints $\mathbf{c}$, we will use the following notational conventions throughout. The asymptotic orbit of $u$ at a puncture $z \in \Gamma^{ \pm}$will be called $\gamma_{z}$, with asymptotic operator $\boldsymbol{A}_{z}:=\boldsymbol{A}_{\gamma_{z}}$, and the collection of these for all punctures will be denoted by $\gamma_{\Gamma}$ and $\boldsymbol{A}_{\Gamma}$ respectively. Denote the corresponding collection of perturbed asymptotic operators $\left\{\boldsymbol{A}_{z} \pm \mathbf{c}_{z}\right\}_{z \in \Gamma^{ \pm}}$by $\boldsymbol{A}_{\Gamma} \pm \mathbf{c}_{\Gamma}$, noting that the sign choice must always match the sign of the puncture. For $i \in\{0,1\}$, let

$$
\Gamma_{i}^{ \pm}(\mathbf{c})=\left\{z \in \Gamma^{ \pm} \mid p\left(\gamma_{z} \pm \mathbf{c}_{z}\right)=i\right\}
$$

and $\Gamma_{i}(\mathbf{c})=\Gamma_{i}^{+}(\mathbf{c}) \cup \Gamma_{i}^{-}(\mathbf{c})$. This defines a partition of $\Gamma$ into so-called even and odd punctures with respect to the constraints. Note that when $\gamma_{z}$ is nondegenerate, the parity of $z$ is simply the even/odd parity of $\mu_{\mathrm{CZ}}^{\Phi}\left(\gamma_{z}\right)$; in general however this distinction depends on not just the orbit and constraints, but also the sign of the puncture.

\footnotetext{
${ }^{6}$ For orbits in two-dimensional families, the numbers $v_{ \pm}(\gamma)$ are closely related to the sign of a Morse-Bott surface, as defined in [Wenb].
} 
Choose $p>2$ and define the Banach manifold

$$
\mathscr{B}:=\mathscr{B}^{1, p, \delta}\left(\dot{\Sigma}, W ; L, P_{\Gamma}\right)
$$

to consist of maps $\dot{\Sigma} \rightarrow W$ of class $W_{\text {loc }}^{1, p}$ which satisfy $u(\partial \Sigma) \subset L$ and have asymptotically cylindrical behavior approaching the orbits $\left\{P_{z}\right\}_{z \in \Gamma}$ at the corresponding punctures: the latter means in particular that using cylindrical coordinates $(s, t) \in Z_{ \pm}$ near $z \in \Gamma^{ \pm}$, there exists an orbit $\gamma_{z} \in P_{z}$ with parametrization $x: \mathbb{R} \rightarrow M_{ \pm}$and a constant $s_{0}$ such that for sufficiently large $|s|$,

$$
u\left(s+s_{0}, t\right)=\exp _{\tilde{x}(s, t)} h(s, t),
$$

where $\tilde{x}(s, t):=\left(T_{z} s, x\left(T_{z} t\right)\right) \in E_{ \pm} \subset \mathbb{R} \times M_{ \pm}$and $h \in \Gamma\left(\tilde{x}^{*} T E_{ \pm}\right)$is of weighted Sobolev class $W^{1, p, \delta}$ on $Z_{ \pm}$. The tangent space $T_{u} \mathcal{B}$ can then be written as

$$
T_{u} \mathcal{B}=W_{\Lambda}^{1, p, \delta}\left(u^{*} T W\right) \oplus V_{\Gamma} \oplus X_{\Gamma},
$$

where the summands are defined as follows. The subscript $\Lambda$ refers to the totally real subbundle

$$
\Lambda:=\left(\left.u\right|_{\partial \Sigma}\right)^{*} T L \rightarrow \partial \Sigma,
$$

so that sections $v \in W_{\Lambda}^{1, p, \delta}\left(u^{*} T W\right)$ are required to satisfy the boundary condition $v(\partial \Sigma) \subset \Lambda$, as well as decaying in accordance with the small exponential weight $\delta>0$ at each end. The other two summands are both finite dimensional vector spaces consisting of sections $\dot{\Sigma} \rightarrow u^{*} T W$ that are supported near infinity and asymptotically equal to constant vectors in some choice of $\mathbb{R}$-invariant coordinates near the asymptotic orbit. In particular, $V_{\Gamma}$ has dimension $2 \# \Gamma$ and contains vector fields that are parallel to the orbit cylinders $\tilde{x}(s, t)=(T s, x(T t))$ near infinity, while the vector fields in $X_{\Gamma}$ are trivial whenever $P_{z}$ is a fixed orbit and otherwise parallel to the Morse-Bott manifolds $P_{z}$, thus

$$
\operatorname{dim} X_{\Gamma}=\sum_{z \in \Gamma}\left(\operatorname{dim} P_{z}-1\right)=\sum_{z \in \Gamma_{U}} \operatorname{dim} \operatorname{ker}\left(\boldsymbol{A}_{z}\right) .
$$

Fixing a complex structure $j$ on $\Sigma$, there is a Banach space bundle $\mathcal{E} \rightarrow \mathscr{B}$ whose fibers are spaces of complex antilinear bundle maps

$$
\varepsilon_{u}=L^{p, \delta}\left(\overline{\operatorname{Hom}}_{\mathbb{C}}\left(T \dot{\Sigma}, u^{*} T W\right)\right),
$$

and the nonlinear Cauchy-Riemann operator defines a smooth section

$$
\bar{\partial}_{J}: \mathscr{B} \rightarrow \mathcal{E}, \quad u \mapsto T u+J \circ T u \circ j,
$$

whose zeroes are parametrizations of asymptotically cylindrical pseudoholomorphic curves $u:(\dot{\Sigma}, j) \rightarrow(W, J)$. The linearization of $\bar{\partial}_{J}$ at a zero $u$ defines a linear 
Cauchy-Riemann type operator,

$$
\begin{aligned}
\boldsymbol{D}_{u}: \Gamma\left(u^{*} T W\right) & \rightarrow \Gamma\left(\overline{\operatorname{Hom}}_{\mathbb{C}}\left(T \dot{\Sigma}, u^{*} T W\right)\right), \\
v & \mapsto \nabla v+J \circ \nabla v \circ j+\left(\nabla_{v} J\right) \circ T u \circ j,
\end{aligned}
$$

where $\nabla$ is any symmetric connection on $W$. As a bounded linear operator $T_{u} \mathscr{B} \rightarrow$ $\mathcal{E}_{u}, \boldsymbol{D}_{u}$ is Fredholm. To write down its index, let $\Phi$ be an arbitrary choice of trivialization for $u^{*} T W$ along $\partial \Sigma$ and for $\xi_{ \pm}$along the orbits $\gamma_{z}$, and define the total Maslov index

$$
\mu^{\Phi}(u ; \mathbf{c})=\mu^{\Phi}\left(u^{*} T W, \Lambda\right)+\sum_{z \in \Gamma^{+}} \mu_{\mathrm{CZ}}^{\Phi}\left(\gamma_{z}+\mathbf{c}_{z}\right)-\sum_{z \in \Gamma^{-}} \mu_{\mathrm{CZ}}^{\Phi}\left(\gamma_{z}-\mathbf{c}_{z}\right) .
$$

The trivializations of $\xi_{ \pm}$extend naturally to trivializations of $T W=T\left(\mathbb{R} \times M_{ \pm}\right)$ along the orbits via the splitting

$$
T\left(\mathbb{R} \times M_{ \pm}\right)=\left(\mathbb{R} \oplus \mathbb{R} X_{ \pm}\right) \oplus \xi_{ \pm},
$$

so that one can also define the relative Chern number $c_{1}^{\Phi}\left(u^{*} T W\right)$.

\section{Proposition 3.7.}

$$
\operatorname{ind}\left(\boldsymbol{D}_{u}\right)=n \chi(\dot{\Sigma})+2 c_{1}^{\Phi}\left(u^{*} T W\right)+\mu^{\Phi}(u ; \mathbf{c})+\# \Gamma .
$$

Proof. Denote by $\boldsymbol{D}_{0}$ the restriction of $\boldsymbol{D}_{u}$ to $W_{\Lambda}^{1, p, \delta}\left(u^{*} T W\right)$, so

$$
\begin{aligned}
\operatorname{ind}\left(\boldsymbol{D}_{u}\right) & =\operatorname{ind}\left(\boldsymbol{D}_{0}\right)+\operatorname{dim} V_{\Gamma}+\operatorname{dim} X_{\Gamma} \\
& =\operatorname{ind}\left(\boldsymbol{D}_{0}\right)+2 \# \Gamma+\sum_{z \in \Gamma_{U}} \operatorname{dim} \operatorname{ker}\left(\boldsymbol{A}_{z}\right) .
\end{aligned}
$$

Then $\boldsymbol{D}_{0}$ is a Cauchy-Riemann type operator asymptotic at $z \in \Gamma$ to the operators

$$
\mathbf{B}_{z}:=\mathbf{C} \oplus \boldsymbol{A}_{z},
$$

where we use the splitting (3.9) and define on the first summand the "trivial" asymptotic operator $\mathbf{C}=-J_{0} \frac{d}{d t}$. The latter is degenerate, but we have

$$
\mu_{\mathrm{CZ}}(\mathbf{C} \pm \boldsymbol{\delta})=\mp 1
$$

if $\boldsymbol{\delta}>0$ is sufficiently small. By the discussion of exponential weights in $\S 2.1, \boldsymbol{D}_{0}$ is now conjugate to a Cauchy-Riemann operator

$$
W_{\Lambda}^{1, p}\left(u^{*} T W\right) \rightarrow L^{p}\left(\overline{\operatorname{Hom}}_{\mathbb{C}}\left(T \dot{\Sigma}, u^{*} T W\right)\right)
$$


with nondegenerate asymptotics, so by (2.1):

$$
\begin{aligned}
\operatorname{ind}\left(\boldsymbol{D}_{0}\right)= & n \chi(\dot{\Sigma})+2 c_{1}^{\Phi}\left(u^{*} T W\right)+\mu^{\Phi}\left(u^{*} T W, \Lambda\right) \\
& +\sum_{z \in \Gamma^{+}} \mu_{\mathrm{CZ}}^{\Phi}\left(\mathbf{B}_{z}+\boldsymbol{\delta}\right)-\sum_{z \in \Gamma^{-}} \mu_{\mathrm{CZ}}^{\Phi}\left(\mathbf{B}_{z}-\boldsymbol{\delta}\right) \\
= & n \chi(\dot{\Sigma})+2 c_{1}^{\Phi}\left(u^{*} T W\right)+\mu^{\Phi}\left(u^{*} T W, \Lambda\right) \\
& +\sum_{z \in \Gamma^{+}} \mu_{\mathrm{CZ}}^{\Phi}\left(\boldsymbol{A}_{z}+\boldsymbol{\delta}\right)-\sum_{z \in \Gamma^{-}} \mu_{\mathrm{CZ}}^{\Phi}\left(\boldsymbol{A}_{z}-\boldsymbol{\delta}\right)-\# \Gamma,
\end{aligned}
$$

where in the last line we have used the splitting $\mathbf{B}_{z}=\mathbf{C} \oplus \boldsymbol{A}_{z}$ and (3.10). Now using (3.3), we have

$$
\begin{aligned}
\sum_{z \in \Gamma^{+}} \mu_{\mathrm{CZ}}^{\Phi}\left(\boldsymbol{A}_{z}+\boldsymbol{\delta}\right)-\sum_{z \in \Gamma^{-}} \mu_{\mathrm{CZ}}^{\Phi}\left(\boldsymbol{A}_{z}-\boldsymbol{\delta}\right)-\# \Gamma & \\
& +2 \# \Gamma+\sum_{z \in \Gamma_{U}} \operatorname{dim} \operatorname{ker}\left(\boldsymbol{A}_{z}\right) \\
= & \sum_{z \in \Gamma_{U}^{+}} \mu_{\mathrm{CZ}}^{\Phi}\left(\boldsymbol{A}_{z}-\boldsymbol{\delta}\right)+\sum_{z \in \Gamma_{C}^{+}} \mu_{\mathrm{CZ}}^{\Phi}\left(\boldsymbol{A}_{z}+\boldsymbol{\delta}\right) \\
& \quad-\sum_{z \in \Gamma_{U}^{-}} \mu_{\mathrm{CZ}}^{\Phi}\left(\boldsymbol{A}_{z}+\boldsymbol{\delta}\right)-\sum_{z \in \Gamma_{C}^{-}} \mu_{\mathrm{CZ}}^{\Phi}\left(\boldsymbol{A}_{z}-\boldsymbol{\delta}\right)+\# \Gamma \\
= & \sum_{z \in \Gamma^{+}} \mu_{\mathrm{CZ}}^{\Phi}\left(\boldsymbol{A}_{z}+\mathbf{c}_{z}\right)-\sum_{z \in \Gamma^{-}} \mu_{\mathrm{CZ}}^{\Phi}\left(\boldsymbol{A}_{z}-\mathbf{c}_{z}\right)+\# \Gamma,
\end{aligned}
$$

and the result follows.

For the following lemma, recall that $\boldsymbol{D}^{\Sigma}: \Gamma(T \dot{\Sigma}) \rightarrow \Gamma\left(\overline{\operatorname{End}}_{\mathbb{C}}(T \dot{\Sigma})\right)$ denotes the natural linear Cauchy-Riemann operator on $\Gamma(T \dot{\Sigma})$ determined by the holomorphic structure of $T \dot{\Sigma}$; it is the linearization at the identity of the operator $\bar{\partial}_{j}^{\Sigma} \varphi=T \varphi+$ $j \circ T \varphi \circ j$ acting on maps $\varphi: \dot{\Sigma} \rightarrow \dot{\Sigma}$. We use the bundle map $d u: T \dot{\Sigma} \rightarrow u^{*} T W$ to define linear maps

$$
\begin{gathered}
\Gamma(T \dot{\Sigma}) \stackrel{d u}{\longrightarrow} \Gamma\left(u^{*} T W\right), \\
\Gamma\left(\overline{\operatorname{End}}_{\mathbb{C}}(T \dot{\Sigma})\right) \stackrel{d u}{\longrightarrow} \Gamma\left(\overline{\operatorname{Hom}}_{\mathbb{C}}\left(T \dot{\Sigma}, u^{*} T W\right) .\right.
\end{gathered}
$$

Lemma 3.8. For any smooth vector field $v \in \Gamma(T \dot{\Sigma})$,

$$
\boldsymbol{D}_{u}(d u(v))=d u\left(\boldsymbol{D}^{\Sigma} v\right)
$$


Proof. Choose any open subset $U \subset \dot{\Sigma}$ with compact support. On this neighborhood, the flow $\varphi^{\tau}$ of $v$ is well defined for $\tau$ sufficiently close to 0 , and by definition, if $z \in \mathcal{U}$ and $Y \in T_{z} \dot{\Sigma}$,

$$
\left(\boldsymbol{D}^{\Sigma} v\right) Y=\left.\nabla_{\tau}\left[\bar{\partial}_{j}^{\Sigma} \varphi^{\tau}(Y)\right]\right|_{\tau=0},
$$

where $\nabla$ is any connection on $\dot{\Sigma}$. Similarly, using the fact that $u:(\dot{\Sigma}, j) \rightarrow(W, J)$ and $\mathbb{1}:(\dot{\Sigma}, j) \rightarrow(\dot{\Sigma}, j)$ are both holomorphic,

$$
\begin{aligned}
\boldsymbol{D}_{u}(d u(v))(Y) & =\left.\nabla_{\tau}\left[\bar{\partial}_{J}\left(u \circ \varphi^{\tau}\right)(Y)\right]\right|_{\tau=0} \\
& =\left.\nabla_{\tau}\left[T\left(u \circ \varphi^{\tau}\right)(Y)+J \circ T\left(u \circ \varphi^{\tau}\right) \circ j(Y)\right]\right|_{\tau=0} \\
& =\left.\nabla_{\tau}\left[T u \circ T \varphi^{\tau}(Y)+J \circ T u \circ T \varphi^{\tau} \circ j(Y)\right]\right|_{\tau=0} \\
& =\left.\nabla_{\tau}\left[T u \circ T \varphi^{\tau}(Y)+T u \circ j \circ T \varphi^{\tau} \circ j(Y)\right]\right|_{\tau=0} \\
& =\left.\nabla_{\tau}\left[d u\left(\varphi^{\tau}(z)\right) \cdot \bar{\partial}_{j}^{\Sigma} \varphi^{\tau}(Y)\right]\right|_{\tau=0} \\
& =\nabla_{v(z)}(d u) \cdot \bar{\partial}_{j}^{\Sigma}(\mathbb{1})(Y)+d u\left(\left.\nabla_{\tau}\left[\bar{\partial}_{j}^{\Sigma} \varphi^{\tau}(Y)\right]\right|_{\tau=0}\right) \\
& =d u\left(D^{\Sigma} v(Y)\right) .
\end{aligned}
$$

Varying complex structures on the domain can be incorporated into the picture by fixing $j_{0} \in \mathcal{L}(\Sigma)$ and choosing a Teichmüller slice $\mathcal{T}$ through $j_{0}$ (see Definition 3.2). We can now redefine the Banach space bundle $\mathcal{E}$ over $\mathcal{T} \times \mathscr{B}$ so that

$$
\varepsilon_{(j, u)}=L^{p, \boldsymbol{\delta}}\left(\overline{\operatorname{Hom}}_{\mathbb{C}}\left((T \dot{\Sigma}, j),\left(u^{*} T W, J\right)\right)\right),
$$

and extend the section $\bar{\partial}_{J}$ over this bundle by

$$
\bar{\partial}_{J}: \mathcal{T} \times \mathscr{B} \rightarrow \mathcal{E}, \quad(j, u) \mapsto T u+J \circ T u \circ j .
$$

The linearization at $(j, u) \in \bar{\partial}_{J}^{-1}(0)$ can now be expressed via its "partial derivatives,"

$$
D \bar{\partial}_{J}(j, u): T_{j} \mathcal{T} \oplus T_{u} \mathscr{B} \rightarrow \mathcal{E}_{(j, u)}, \quad(y, v) \mapsto \boldsymbol{G}_{u} y+\boldsymbol{D}_{u} v
$$

where

$$
\begin{aligned}
\boldsymbol{G}_{u}: T_{j} \mathcal{T} \subset \Gamma\left(\overline{\operatorname{End}}_{\mathbb{C}}(T \dot{\Sigma})\right) & \rightarrow \Gamma\left(\overline{\operatorname{Hom}}_{\mathbb{C}}\left((T \dot{\Sigma}, j),\left(u^{*} T W, J\right)\right)\right) \\
y & \mapsto J \circ T u \circ y .
\end{aligned}
$$

We can now present the precise definition of regularity.

Definition 3.9. The curve $(\Sigma, j, \Gamma, u) \in \mathcal{M}^{\mathbf{c}}$ is called regular if there exists a Teichmüller slice $\mathcal{T}$ through $j$ such that the operator $D \bar{\partial}_{J}(j, u): T_{j} \mathcal{T} \oplus T_{u} \mathcal{B} \rightarrow \mathcal{E}_{(j, u)}$ is surjective. 
Remark 3.10. This condition clearly does not depend on the choice of map $u:(\dot{\Sigma}, j) \rightarrow(W, J)$ representing a given equivalence class in $\mathcal{M}^{\mathbf{c}} ;$ if $\varphi:\left(\Sigma^{\prime}, j^{\prime}\right) \rightarrow$ $(\Sigma, j)$ is a biholomorphic map and $u^{\prime}=u \circ \varphi$, one can use the pullback $\varphi^{*}$ to construct a Teichmüller slice $\overline{\mathcal{T}}^{\prime}$ through $j^{\prime}$ so that the operators $D \bar{\partial}_{J}\left(j^{\prime}, u^{\prime}\right)$ and $D \bar{\partial}_{J}(j, u)$ are conjugate. The next lemma shows also that the surjectivity of $D \bar{\partial}_{J}(j, u)-$ and in fact the codimension of its image - does not depend on the choice of Teichmüller slice.

Lemma 3.11. For $(\Sigma, j, \Gamma, u) \in \mathcal{M}^{\mathbf{c}}$ and any two Teichmüller slices $\mathcal{T}$ and $\mathcal{T}^{\prime}$ through $j$, denote by $\boldsymbol{L}: T_{j} \mathcal{T} \oplus T_{u} \mathcal{B} \rightarrow \mathcal{E}_{(j, u)}$ and $\boldsymbol{L}^{\prime}: T_{j} \mathcal{T}^{\prime} \oplus T_{u} \mathscr{B} \rightarrow \mathcal{E}_{(j, u)}$ the corresponding linearizations of $\bar{\partial}_{J}$ at $(j, u)$. Then $\operatorname{im}(\boldsymbol{L})=\operatorname{im}\left(\boldsymbol{L}^{\prime}\right)$.

Proof. Using the inclusion $T_{j} \mathcal{T} \subset L^{p, \boldsymbol{\delta}}\left(\overline{\operatorname{End}}_{\mathbb{C}}(T \dot{\Sigma})\right)$, extend $\boldsymbol{L}$ to

$$
\begin{aligned}
\overline{\boldsymbol{L}}: L^{p, \delta}\left(\overline{\operatorname{End}}_{\mathbb{C}}(T \dot{\Sigma})\right) \oplus T_{u} \mathscr{B} & \rightarrow \mathcal{E}_{(j, u)} \\
(y, v) & \mapsto J \circ T u \circ y+\boldsymbol{D}_{u} v .
\end{aligned}
$$

For $y=\boldsymbol{D}^{\Sigma} Y \in \operatorname{im}\left(\boldsymbol{D}^{\Sigma}\right) \subset L^{p, \boldsymbol{\delta}}\left(\overline{\operatorname{End}}_{\mathbb{C}}(T \dot{\Sigma})\right)$, we use the fact that $u$ is $J$ holomorphic and write

$$
\overline{\boldsymbol{L}}(y, 0)=J \circ T u \circ y=d u(j y)=d u\left(\boldsymbol{D}^{\Sigma}(j Y)\right) .
$$

Then by Lemma 3.8, this equals $\boldsymbol{D}_{u}(d u(j Y))$ if $Y$ is smooth, and the same holds for general $Y$ in the domain of $\boldsymbol{D}^{\Sigma}$ by a density argument, thus the restriction of $\overline{\boldsymbol{L}}$ to $\operatorname{im}\left(\boldsymbol{D}^{\Sigma}\right)$ has image contained in im $\left(\boldsymbol{D}_{u}\right)$. Since $L^{p, \boldsymbol{\delta}}\left(\overline{\operatorname{End}}_{\mathbb{C}}(T \dot{\Sigma})\right)=\operatorname{im}\left(\boldsymbol{D}^{\Sigma}\right) \oplus T_{j} \mathcal{T}$ by Corollary 3.6, this implies $\operatorname{im}(\overline{\boldsymbol{L}})=\operatorname{im}(\boldsymbol{L})$. Now using the same argument for $\mathcal{T}^{\prime}$, we have $\operatorname{im}(\boldsymbol{L})=\operatorname{im}(\overline{\boldsymbol{L}})=\operatorname{im}\left(\boldsymbol{L}^{\prime}\right)$.

Since $\boldsymbol{D}_{u}$ is Fredholm and $\mathcal{T}$ is finite dimensional, $D \bar{\partial}_{J}(j, u)$ is also Fredholm. Recalling (3.1) and the definition of $\operatorname{ind}(u ; \mathbf{c})=\operatorname{vir}-\operatorname{dim} \mathcal{M}_{u}^{\mathbf{c}}$ in (1.1), we have

$$
\text { ind } D \bar{\partial}_{J}(j, u)=\operatorname{dim} \mathcal{T}+\operatorname{ind}\left(\boldsymbol{D}_{u}\right)=\operatorname{ind}(u ; \mathbf{c})+\operatorname{dim} \operatorname{Aut}(\dot{\Sigma}, j) \text {. }
$$

For completeness, we now prove the fact that regularity gives $\mathcal{M}^{\mathbf{c}}$ the structure of a smooth orbifold of dimension ind $(u ; \mathbf{c})$ near $u$.

Proof of Theorem 0. Assume $\left(j_{0}, u_{0}\right) \in \bar{\partial}_{J}^{-1}(0)$ is regular and let $G=\operatorname{Aut}\left(\dot{\Sigma}, j_{0}\right)$. By Lemma 3.11, the regularity condition is independent of the choice of Teichmüller slice, so if $\dot{\Sigma}$ is stable, then using Lemma 3.3 we can pick a slice $\mathcal{T}$ through $j_{0}$ that is invariant under the natural $G$-action. Similarly if $\dot{\Sigma}$ is $\mathbb{A}$ or $\mathbb{T}^{2}$, then without loss of generality we can compose with a diffeomorphism such that $j_{0}$ belongs to one of the special Teichmüller slices $\mathcal{T}_{\mathbb{A}}$ or $\mathcal{T}_{\mathbb{T}}$ constructed in $\S 3.1$ (which also admit a natural $G$-action), and choose this for $\mathcal{T}$. There is now a $G$-action on $\mathcal{T} \times \mathscr{B}$ defined by

$$
\varphi \cdot(j, u)=\left(\varphi^{*} j, u \circ \varphi\right) .
$$


This clearly preserves $\bar{\partial}_{J}^{-1}(0)$, and the stabilizer of any $(j, u) \in \bar{\partial}_{J}^{-1}(0)$ is the finite subgroup $\left\{\varphi \in \operatorname{Aut}\left(\dot{\Sigma}, j_{0}\right) \mid \varphi^{*} j=j, u \circ \varphi=u\right\} \subset \operatorname{Aut}(u)$. Since $D \bar{\partial}_{J}\left(j_{0}, u_{0}\right)$ is surjective, Remark 3.10 implies that the same is true for all $(j, u)$ in the $G$-orbit of $\left(j_{0}, u_{0}\right)$, thus by the implicit function theorem, a neighborhood $U \subset \bar{\partial}_{J}^{-1}(0)$ of this orbit admits a natural smooth manifold structure, with dimension ind $\left(u_{0} ; \mathbf{c}\right)+$ $\operatorname{dim} \operatorname{Aut}\left(\dot{\Sigma}, j_{0}\right)$. Starting from a small neighborhood of $\left(j_{0}, u_{0}\right)$ in $\bar{\partial}_{J}^{-1}(0)$ and extending this under the $G$-action, we may assume $U$ to be $G$-invariant. The quotient $U / G$ then inherits the structure of a smooth orbifold of dimension ind $\left(u_{0} ; \mathbf{c}\right)$, with isotropy group $\operatorname{Aut}\left(u_{0}\right)$ at $\left(j_{0}, u_{0}\right)$ and a natural isomorphism

$$
T_{\left(j_{0}, u_{0}\right)}(U / G)=\operatorname{ker} D \bar{\partial}_{J}\left(j_{0}, u_{0}\right) / \operatorname{aut}\left(\dot{\Sigma}, j_{0}\right) .
$$

One can adapt the argument in [Dra04] to show that charts constructed in this way are always smoothly compatible.

To complete the proof, we show that $U / G$ is homeomorphic to a neighborhood of $\left(\Sigma, j_{0}, \Gamma, u_{0}\right)$ in $\mathcal{M}$. Clearly $U$ contains a representative of every $J$-holomorphic curve near $\left(j_{0}, u_{0}\right)$, so the point is to show that any two such curves $(j, u)$ and $\left(j^{\prime}, u^{\prime}\right)$ that are equivalent in $\mathcal{M}^{\mathbf{c}}$ are related by the $G$-action.

Suppose first that $\dot{\Sigma}$ is non-stable and is not $\mathbb{A}$ or $\mathbb{T}^{2}$ : then $\mathcal{T}$ contains only $j_{0}$, and $\left(j_{0}, u\right) \sim\left(j_{0}, u^{\prime}\right)$ if and only if $u^{\prime}=u \circ \varphi$ for some $\varphi \in \operatorname{Aut}\left(\dot{\Sigma}, j_{0}\right)=G$, so we are done. The case $\mathbb{A}$ is hardly more complicated: now $\mathcal{T}$ is 1-dimensional and $M(\dot{\Sigma})$ is trivial, so $\mathcal{M}(\dot{\Sigma})=\mathcal{T}(\dot{\Sigma})$ and $j, j^{\prime} \in \mathcal{T}$ are equivalent in $\mathcal{M}(\dot{\Sigma})$ if and only if $j=j^{\prime}$. Thus $(j, u) \sim\left(j^{\prime}, u^{\prime}\right)$ implies $j=j^{\prime}$ and $u^{\prime}=u \circ \varphi$ for some $\varphi \in \operatorname{Aut}(\dot{\Sigma}, j)$. But our construction of $\mathcal{J}_{\mathbb{A}}$ identifies $\operatorname{Aut}(\dot{\Sigma}, j)$ with $\operatorname{Aut}\left(\dot{\Sigma}, j_{0}\right)=G$, so again we are done.

Consider now the stable cases and $\mathbb{T}^{2}$, for which $M(\dot{\Sigma})$ is nontrivial. For these, the groups $\operatorname{Aut}_{0}(\dot{\Sigma}, j):=\operatorname{Aut}(\dot{\Sigma}, j) \cap \operatorname{Diff}_{0}(\Sigma, \Gamma)$ for every $j \in \mathcal{T}$ are identified with $\operatorname{Aut}_{0}\left(\dot{\Sigma}, j_{0}\right)$; this is a nontrivial statement only for $\dot{\Sigma}=\mathbb{T}^{2}$, where our explicit construction of $\mathcal{T}=\mathcal{T}_{\mathbb{T}^{2}}$ identifies every $\operatorname{Aut}_{0}\left(\mathbb{T}^{2}, j\right)$ with $\mathbb{T}^{2}$, acting by translations. Meanwhile, for each $j \in \mathcal{T}$ there is a finite subgroup $G_{j} \subset \operatorname{Aut}(\dot{\Sigma}, j)$ $\left(G_{j}=\operatorname{Aut}(\dot{\Sigma}, j)\right.$ in the stable cases) naturally isomorphic to the stabilizer of $[j] \in \mathcal{T}(\dot{\Sigma})$ under the $M(\dot{\Sigma})$-action, such that $\operatorname{Aut}(\dot{\Sigma}, j)$ is the semidirect product of $G_{j}$ with $\operatorname{Aut}_{0}\left(\dot{\Sigma}, j_{0}\right)$. Now if $(j, u)$ and $\left(j^{\prime}, u^{\prime}\right)$ are two elements of $\mathcal{U}$ that represent equivalent curves, so $j^{\prime}=\psi^{*} j$ and $u^{\prime}=u \circ \psi$ for some $\psi \in \operatorname{Diff}_{+}(\Sigma, \Gamma)$, we need to show that $\psi \in G$. In terms of the $M(\dot{\Sigma})$-action on $\mathcal{T}(\dot{\Sigma}),[\psi] \cdot[j]=\left[j^{\prime}\right]$ implies that if $j$ and $j^{\prime}$ are both sufficiently close to $j_{0}$, then $[\psi]$ belongs to the stabilizer of $\left[j_{0}\right]$, i.e. $[\psi] \cdot\left[j_{0}\right]=\left[j_{0}\right]$. Thus there is a unique $\varphi \in G_{j_{0}}$ such that $[\varphi]=[\psi] \in M(\dot{\Sigma})$, and by construction, $\varphi^{*} j=j^{\prime}$. It follows that $\left(\psi \circ \varphi^{-1}\right)^{*} j=j$, so $\psi \circ \varphi^{-1} \in \operatorname{Aut}_{0}(\dot{\Sigma}, j)=\operatorname{Aut}_{0}\left(\dot{\Sigma}, j_{0}\right)$, and $\psi$ is thus a product of two maps in $\operatorname{Aut}\left(\dot{\Sigma}, j_{0}\right)$. 
3.3. The generalized normal bundle. For the remainder of this section, we shall consider a fixed non-constant holomorphic curve $(j, u) \in \bar{\partial}_{J}^{-1}(0) \subset \mathcal{T} \times \mathscr{B}$ and examine the operator $D \bar{\partial}_{J}(j, u)=\boldsymbol{G}_{u}+\boldsymbol{D}_{u}$ more closely. When we refer to $\dot{\Sigma}$ or $\Sigma$ as a Riemann surface, we will always mean with complex structure $j$.

As was observed in [IS99], the operator $\boldsymbol{D}_{u}$ defines a natural holomorphic structure on the bundle $u^{*} T W \rightarrow \dot{\Sigma}$ : indeed, the complex linear part of $\boldsymbol{D}_{u}$ is also a Cauchy-Riemann type operator, so there is a unique holomorphic structure whose local holomorphic sections vanish under this operator. This induces a holomorphic structure on $\operatorname{Hom}_{\mathbb{C}}\left(T \dot{\Sigma}, u^{*} T W\right)$, and one can then show (cf. [IS99]) that

$$
d u \in \Gamma\left(\operatorname{Hom}_{\mathbb{C}}\left(T \dot{\Sigma}, u^{*} T W\right)\right)
$$

is a holomorphic section. Thus if $z_{0}$ is an interior critical point of $u$, we can choose a holomorphic trivialization of $\operatorname{Hom}_{\mathbb{C}}\left(T \dot{\Sigma}, u^{*} T W\right)$ near $z_{0}$ and express $d u$ as a $\mathbb{C}^{n}$-valued function of the form

$$
\left(z-z_{0}\right)^{k} F(z)
$$

for some $k \in \mathbb{N}$ and $\mathbb{C}^{n}$-valued holomorphic function $F$ with $F\left(z_{0}\right) \neq 0$. In this case we define the order of the critical point by

$$
\operatorname{ord}\left(d u ; z_{0}\right)=k .
$$

A similar definition is possible for $z_{0} \in \operatorname{Crit}(u) \cap \partial \Sigma$ since $d u$ satisfies the totally real boundary condition $d u(\partial \Sigma) \subset \mathscr{L}$, where

$$
\mathscr{L}=\left\{\left.A \in \operatorname{Hom}_{\mathbb{C}}\left(T \dot{\Sigma}, u^{*} T W\right)\right|_{\partial \Sigma} \mid A(T(\partial \Sigma)) \subset \Lambda\right\} .
$$

Indeed, one can then choose a trivialization near $z_{0}$ such that $d u$ satisfies the Schwartz reflection principal, and define ord $\left(d u ; z_{0}\right)$ again via (3.13) after reflection. Define the $\frac{1}{2} \mathbb{Z}$-valued algebraic count of critical points by

$$
Z(d u)=\sum_{z \in d u^{-1}(0) \cap \operatorname{int} \dot{\Sigma}} \operatorname{ord}(d u ; z)+\frac{1}{2} \sum_{z \in d u^{-1}(0) \cap \partial \Sigma} \operatorname{ord}(d u ; z) .
$$

The expression (3.13) has a second important purpose: the complex subspace of $T_{u(z)} W$ spanned in the trivialization by $F(z) \in \mathbb{C}^{n} \backslash\{0\}$ allows us to define a smooth rank 1 subbundle

$$
T_{u} \subset u^{*} T W
$$

such that for any $z \in \dot{\Sigma} \backslash \operatorname{Crit}(u),\left(T_{u}\right)_{z}=\operatorname{im} d u(z)$. We will call this the generalized tangent bundle to $u$.

Lemma 3.12. The intersection $\left(T_{u}\right)_{z} \cap \Lambda_{z}$ is 1-dimensional for all $z \in \partial \Sigma$. 
Proof. It can never be 2-dimensional since $\left(T_{u}\right)_{z}$ is a complex subspace and $\Lambda_{z}$ is totally real. Moreover, it is clearly at least 1-dimensional whenever $d u(z) \neq 0$, as then $T u(Y) \in T_{u(z)} L=\Lambda_{z}$ for any $Y \in T_{z} \partial \Sigma$. Since critical points are isolated and the condition $\operatorname{dim}\left(T_{u}\right)_{z} \cap \Lambda_{z}=0$ is open, the result follows.

By the lemma, we can define a totally real subbundle

$$
\ell^{T}=\left.\left.\Lambda \cap T_{u}\right|_{\partial \Sigma} \subset T_{u}\right|_{\partial \Sigma},
$$

and by construction $d u$ now defines a section of the complex line bundle

$$
\operatorname{Hom}_{\mathbb{C}}\left(T \dot{\Sigma}, T_{u}\right) \rightarrow \dot{\Sigma}
$$

with totally real boundary condition $d u(\partial \Sigma) \subset \mathscr{L}^{T}$, where

$$
\mathscr{L}^{T}=\left\{\left.A \in \operatorname{Hom}_{\mathbb{C}}\left(T \dot{\Sigma}, T_{u}\right)\right|_{\partial \Sigma} \mid A(T(\partial \Sigma)) \subset \ell^{T}\right\} .
$$

As defined in the appendix, the algebraic count of zeroes for this section is precisely $Z(d u)$.

Observe that both $T \dot{\Sigma}$ and $T_{u}$ admit natural extensions over the compactified surface $\bar{\Sigma}$; we define this extension for $T_{u}$ via its natural identification with $T \dot{\Sigma}$ under $d u$ since $u$ is immersed near infinity. There is also a natural trivialization $\tau$ of $T \dot{\Sigma}$ at infinity defined by the cylindrical coordinates $(s, t) \in Z_{ \pm}$, and we can define $\tau$ also over $\partial \Sigma$ such that the Maslov index $\mu^{\tau}(T \dot{\Sigma}, T(\partial \Sigma))$ vanishes. Then

$$
c_{1}^{\tau}(T \dot{\Sigma})=\chi(\dot{\Sigma}) \text {. }
$$

Now choose any trivialization $\Phi$ of $T_{u}$ over $\partial \Sigma$ and define it at infinity to be the same as $\tau$. The combination of $\tau$ and $\Phi$ induces a trivialization of $\operatorname{Hom}_{\mathbb{C}}\left(T \dot{\Sigma}, T_{u}\right)$ over $\partial \Sigma$ and at infinity, which we will also denote by $\Phi$. Then we can apply Proposition A.2, noting that the winding terms are zero by construction, and obtain

$$
Z(d u)=c_{1}^{\Phi}\left(\operatorname{Hom}_{\mathbb{C}}\left(T \dot{\Sigma}, T_{u}\right)\right)+\frac{1}{2} \mu^{\Phi}\left(\operatorname{Hom}_{\mathbb{C}}\left(T \dot{\Sigma}, T_{u}\right), \mathscr{L}^{T}\right) .
$$

To break this down further, note that the natural bundle isomorphism

$$
T \dot{\Sigma} \otimes \operatorname{Hom}_{\mathbb{C}}\left(T \dot{\Sigma}, T_{u}\right) \rightarrow T_{u}, \quad v \otimes A \mapsto A v,
$$

sends $T(\partial \Sigma) \otimes \mathscr{L}^{T}$ to $\ell^{T}$, thus

$$
c_{1}^{\Phi}\left(T_{u}\right)=c_{1}^{\tau}(T \dot{\Sigma})+c_{1}^{\Phi}\left(\operatorname{Hom}_{\mathbb{C}}\left(T \dot{\Sigma}, T_{u}\right)\right)=\chi(\dot{\Sigma})+c_{1}^{\Phi}\left(\operatorname{Hom}_{\mathbb{C}}\left(T \dot{\Sigma}, T_{u}\right)\right),
$$

and

$$
\begin{aligned}
\mu^{\Phi}\left(T_{u}, \ell^{T}\right) & =\mu^{\tau}(T \dot{\Sigma}, T(\partial \Sigma))+\mu^{\Phi}\left(\operatorname{Hom}_{\mathbb{C}}\left(T \dot{\Sigma}, T_{u}\right), \mathscr{L}^{T}\right) \\
& =\mu^{\Phi}\left(\operatorname{Hom}_{\mathbb{C}}\left(T \dot{\Sigma}, T_{u}\right), \mathscr{L}^{T}\right),
\end{aligned}
$$


so (3.15) implies

$$
c_{1}^{\Phi}\left(T_{u}\right)=\chi(\dot{\Sigma})-\frac{1}{2} \mu^{\Phi}\left(T_{u}, \ell^{T}\right)+Z(d u) .
$$

We next choose a generalized normal bundle $N_{u} \rightarrow \dot{\Sigma}$, which we define to be any rank $n-1$ subbundle of $u^{*} T W$ such that

$$
u^{*} T W=T_{u} \oplus N_{u},
$$

and the following conditions are satisfied:

(1) On the cylindrical neighborhoods $U_{z}$ for $z \in \Gamma^{ \pm}, N_{u}$ matches the hyperplane distributions $\xi_{ \pm}$, and thus extends to infinity as $\left.N_{u}\right|_{\delta_{z}}=\left.\xi_{ \pm}\right|_{\gamma_{z}}$.

(2) For $z \in \partial \Sigma$, there is always a real $(n-1)$-dimensional intersection $\ell_{z}^{N}:=$ $\left(N_{u}\right)_{z} \cap \Lambda_{z}$, thus defining a totally real subbundle

$$
\left.\ell^{N} \subset N_{u}\right|_{\partial \Sigma}
$$

such that $\ell^{T} \oplus \ell^{N}=\Lambda$.

3.4. Splitting the linearization. The splitting $u^{*} T W=T_{u} \oplus N_{u}$ defines projection maps $\pi_{T} \in \Gamma\left(\operatorname{Hom}_{\mathbb{C}}\left(u^{*} T W, T_{u}\right)\right)$ and $\pi_{N} \in \Gamma\left(\operatorname{Hom}_{\mathbb{C}}\left(u^{*} T W, N_{u}\right)\right)$, both of which are smooth and satisfy exponential decay conditions due to the asymptotic behavior of $u$. It follows that these define bounded linear projection operators

$$
\begin{aligned}
& W_{\Lambda}^{1, p, \boldsymbol{\delta}}\left(u^{*} T W\right) \oplus V_{\Gamma} \oplus X_{\Gamma} \stackrel{\pi_{T}}{\longrightarrow} W_{\ell^{T}}^{1, p, \boldsymbol{\delta}}\left(T_{u}\right) \oplus V_{\Gamma}^{T}, \\
& W_{\Lambda}^{1, p, \boldsymbol{\delta}}\left(u^{*} T W\right) \oplus V_{\Gamma} \oplus X_{\Gamma} \stackrel{\pi_{N}}{\longrightarrow} W_{\ell^{N}}^{1, p, \boldsymbol{\delta}}\left(N_{u}\right) \oplus X_{\Gamma},
\end{aligned}
$$

where $V_{\Gamma}^{T} \subset \Gamma\left(T_{u}\right)$ is the isomorphic image of $V_{\Gamma}^{\Sigma} \subset \Gamma(T \dot{\Sigma})$ under the map $d u: \Gamma(T \dot{\Sigma}) \rightarrow \Gamma\left(T_{u}\right), v \mapsto T u \circ v$, and without loss of generality $X_{\Gamma} \in \Gamma\left(N_{u}\right)$. There is thus a Banach space splitting

$$
W_{\Lambda}^{1, p, \delta}\left(u^{*} T W\right) \oplus V_{\Gamma} \oplus X_{\Gamma}=\left(W_{\ell^{T}}^{1, p, \delta}\left(T_{u}\right) \oplus V_{\Gamma}^{T}\right) \oplus\left(W_{\ell^{N}}^{1, p, \delta}\left(N_{u}\right) \oplus X_{\Gamma}\right),
$$

and a similar splitting

$$
L^{p, \delta}\left(\overline{\operatorname{Hom}}_{\mathbb{C}}\left(T \dot{\Sigma}, u^{*} T W\right)\right)=L^{p, \delta}\left(\overline{\operatorname{Hom}}_{\mathbb{C}}\left(T \dot{\Sigma}, T_{u}\right)\right) \oplus L^{p, \delta}\left(\overline{\operatorname{Hom}}_{\mathbb{C}}\left(T \dot{\Sigma}, N_{u}\right)\right),
$$

so that with respect to these splittings, the operator

$$
\boldsymbol{D}_{u}: W_{\Lambda}^{1, p, \delta}\left(u^{*} T W\right) \oplus V_{\Gamma} \oplus X_{\Gamma} \rightarrow L^{p, \delta}\left(\overline{\operatorname{Hom}}_{\mathbb{C}}\left(T \dot{\Sigma}, u^{*} T W\right)\right)
$$

can be written in matrix form as

$$
\boldsymbol{D}_{u}=\left(\begin{array}{cc}
\boldsymbol{D}_{u}^{T} & \boldsymbol{D}_{u}^{N T} \\
\boldsymbol{D}_{u}^{T N} & \boldsymbol{D}_{u}^{N}
\end{array}\right)
$$


It is trivial to show that

$$
\boldsymbol{D}_{u}^{T}: W_{\ell^{T}}^{1, p, \boldsymbol{\delta}}\left(T_{u}\right) \oplus V_{\Gamma}^{T} \rightarrow L^{p, \boldsymbol{\delta}}\left(\overline{\operatorname{Hom}}_{\mathbb{C}}\left(T \dot{\Sigma}, T_{u}\right)\right)
$$

and

$$
\boldsymbol{D}_{u}^{N}: W_{\ell^{N}}^{1, p, \boldsymbol{\delta}}\left(N_{u}\right) \oplus X_{\Gamma} \rightarrow L^{p, \boldsymbol{\delta}}\left(\overline{\operatorname{Hom}}_{\mathbb{C}}\left(T \dot{\Sigma}, N_{u}\right)\right)
$$

each satisfy the appropriate Leibnitz rule for a Cauchy-Riemann type operator. The former is asymptotic at each puncture $z \in \Gamma^{ \pm}$to the degenerate asymptotic operator $-J_{0} \frac{d}{d t}$ on a trivial complex line bundle; removing the exponential weight as in $\S 2.1$, this operator becomes $-J_{0} \frac{d}{d t} \pm \delta$, giving Conley-Zehnder index $\mp 1$ with respect to the natural trivialization $\tau$. Thus the restriction of $\boldsymbol{D}_{u}^{T}$ to $W_{\ell^{T}}^{1, p, \delta}\left(T_{u}\right)$ has index

$$
\chi(\dot{\Sigma})+2 c_{1}^{\Phi}\left(T_{u}\right)+\mu^{\Phi}\left(T_{u}, \ell^{T}\right)-\# \Gamma
$$

and adding the dimension of $V_{\Gamma}^{T}$ we find

$$
\begin{aligned}
\operatorname{ind}\left(\boldsymbol{D}_{u}^{T}\right) & =\chi(\dot{\Sigma})+2 c_{1}^{\Phi}\left(T_{u}\right)+\mu^{\Phi}\left(T_{u}, \ell^{T}\right)+\# \Gamma \\
& =3 \chi(\dot{\Sigma})+\# \Gamma+2 Z(d u) \\
& =\operatorname{dim} \operatorname{Aut}(\dot{\Sigma}, j)-\operatorname{dim} \mathcal{T}(\dot{\Sigma})+2 Z(d u),
\end{aligned}
$$

where the second line follows from (3.16).

We call $\boldsymbol{D}_{u}^{N}$ the normal Cauchy-Riemann operator at $u$. It is also Fredholm; from the asymptotic identification of $N_{u}$ with $\xi_{ \pm}$along orbits, we see that $\boldsymbol{D}_{u}^{N}$ is asymptotic to $\boldsymbol{A}_{z}$ at each puncture $z \in \Gamma$. We can use (3.16) to relate its index to ind $(u ; \mathbf{c})$. Abbreviate $\mu_{\mathrm{CZ}}^{\Phi}\left(\gamma_{\Gamma} \pm \mathbf{c}_{\Gamma}\right)=\sum_{z \in \Gamma^{+}} \mu_{\mathrm{CZ}}^{\Phi}\left(\gamma_{z}+\mathbf{c}_{z}\right)-\sum_{z \in \Gamma^{-}} \mu_{\mathrm{CZ}}^{\Phi}\left(\gamma_{z}-\mathbf{c}_{z}\right)$. Then removing the exponential weights as in $\$ 2.1$, we apply the Riemann-Roch formula (2.1) and repeat the calculation in (3.11) to find

$$
\begin{aligned}
\operatorname{ind}\left(\boldsymbol{D}_{u}^{N}\right)= & (n-1) \chi(\dot{\Sigma})+2 c_{1}^{\Phi}\left(N_{u}\right)+\mu^{\Phi}\left(N_{u}, \ell^{N}\right) \\
& +\sum_{z \in \Gamma^{+}} \mu_{\mathrm{CZ}}^{\Phi}\left(\boldsymbol{A}_{z}+\boldsymbol{\delta}\right)-\sum_{z \in \Gamma^{-}} \mu_{\mathrm{CZ}}^{\Phi}\left(\boldsymbol{A}_{z}-\boldsymbol{\delta}\right)+\operatorname{dim} X_{\Gamma} \\
= & (n-1) \chi(\dot{\Sigma})+2 c_{1}^{\Phi}\left(N_{u}\right)+\mu^{\Phi}\left(N_{u}, \ell^{N}\right)+\mu_{\mathrm{CZ}}^{\Phi}\left(\gamma_{\Gamma} \pm \mathbf{c}_{z}\right) \\
= & (n-1) \chi(\dot{\Sigma})+2\left[c_{1}^{\Phi}\left(u^{*} T W\right)-c_{1}^{\Phi}\left(T_{u}\right)\right] \\
& \quad+\left[\mu^{\Phi}\left(u^{*} T W, \Lambda\right)-\mu^{\Phi}\left(T_{u}, \ell^{T}\right)\right]+\mu_{\mathrm{CZ}}^{\Phi}\left(\gamma_{\Gamma} \pm \mathbf{c}_{\Gamma}\right) \\
= & (n-1) \chi(\dot{\Sigma})+2 c_{1}^{\Phi}\left(u^{*} T W\right)-2 \chi(\dot{\Sigma})-2 Z(d u)+\mu^{\Phi}(u ; \mathbf{c}) \\
= & \operatorname{ind}(u ; \mathbf{c})-2 Z(d u) .
\end{aligned}
$$

The main goal for this section is the following: 
Theorem 3. Assume $(\Sigma, j, \Gamma, u) \in \mathcal{M}^{\mathbf{c}}$ is a non-constant curve with Morse-Bott asymptotic orbits and $\mathcal{T}$ is any Teichmüller slice through $j$. Then $\operatorname{ker} D \bar{\partial}_{J}(j, u)$ contains a subspace $\operatorname{ker}\left(\boldsymbol{G}_{u}+\boldsymbol{D}_{u}^{T}\right) \subset T_{j} \mathcal{T} \oplus W_{\ell T}^{1, p, \boldsymbol{\delta}}\left(T_{u}\right) \oplus V_{\Gamma}^{T}$ of dimension $2 Z(d u)+\operatorname{dim} \operatorname{Aut}(\dot{\Sigma}, j)$ such that the normal projection induces a natural isomorphism

$$
\operatorname{ker} D \bar{\partial}_{J}(j, u) / \operatorname{ker}\left(\boldsymbol{G}_{u}+\boldsymbol{D}_{u}^{T}\right)=\operatorname{ker} \boldsymbol{D}_{u}^{N},
$$

and

$$
\operatorname{im} D \bar{\partial}_{J}(j, u)=L^{p, \delta}\left(\overline{\operatorname{Hom}}_{\mathbb{C}}\left(T \dot{\Sigma}, T_{u}\right)\right) \oplus \operatorname{im} \boldsymbol{D}_{u}^{N} .
$$

In particular, we have

$$
\begin{aligned}
\operatorname{dim} \operatorname{ker} D \bar{\partial}_{J}(j, u) & =2 Z(d u)+\operatorname{dim} \operatorname{Aut}(\dot{\Sigma}, j)+\operatorname{dim} \operatorname{ker} \boldsymbol{D}_{u}^{N}, \\
\operatorname{dim} \operatorname{coker} D \bar{\partial}_{J}(j, u) & =\operatorname{dim} \operatorname{coker} \boldsymbol{D}_{u}^{N} .
\end{aligned}
$$

Corollary 3.13. $(\Sigma, j, \Gamma, u) \in \mathcal{M}^{\mathbf{c}}$ is regular if and only if $\boldsymbol{D}_{u}^{N}$ is surjective.

The reason for this result is essentially that the analysis of the map $(y, v) \mapsto$ $\boldsymbol{G}_{u} y+\boldsymbol{D}_{u} v$ when $v$ is a section of $T_{u}$ can be reduced to Lemma 3.5, which one can regard as an analytical statement about the smoothness of Teichmüller space. To achieve this reduction, we introduce certain special Banach spaces of sections: for each $z_{0} \in \operatorname{Crit}(u)$, choose holomorphic coordinates and corresponding trivializations of $T \dot{\Sigma}$ and $T_{u}$ near $z_{0}$ so that the bundle map $d u: T \dot{\Sigma} \rightarrow T_{u}$ locally takes the form $z \mapsto z^{k}$, where $k=\operatorname{ord}\left(d u ; z_{0}\right)$. Now for any function $d: \operatorname{Crit}(u) \rightarrow \mathbb{Z}$, define the Banach space

$$
W^{k, p, \delta, d}(T \dot{\Sigma})
$$

to consist of sections $v$ that are of class $W_{\text {loc }}^{k, p}$ on $\dot{\Sigma} \backslash \operatorname{Crit}(u)$, class $W^{k, p, \delta}$ near infinity, and such that near each $z_{0} \in \operatorname{Crit}(u)$, using the coordinates and trivialization chosen above, the map

$$
z^{d\left(z_{0}\right)} v(z)
$$

is of class $W^{k, p}$. Note that $v\left(z_{0}\right)$ may or may not be well defined: if $d\left(z_{0}\right)>0$ then $v$ is allowed to blow up at $z_{0}$, e.g. it could be meromorphic with a pole of order $\leq d\left(z_{0}\right)$. A suitable Banach space norm can be defined using weighting functions supported near Crit $(u)$, and the subspace $W_{T(\partial \Sigma)}^{k, p, \delta}(T \dot{\Sigma})$ is defined by adding the usual boundary condition; similarly we can define such spaces on the bundles $T_{u}, \overline{\operatorname{End}}_{\mathbb{C}}(T \dot{\Sigma})$ and $\overline{\operatorname{Hom}}_{\mathbb{C}}\left(T \dot{\Sigma}, T_{u}\right)$. These are naturally isomorphic to our original Banach spaces if $d(z)=0$ for all $z \in \operatorname{Crit}(u)$. 
The usefulness of this notion lies in the fact that if we choose $d(z):=\operatorname{ord}(d u ; z)$, then the correspondence $v \mapsto T u \circ v$ defines Banach space isomorphisms

$$
\begin{gathered}
W_{T(\partial \Sigma)}^{1, p, \delta, d}(T \dot{\Sigma}) \stackrel{d u}{\longrightarrow} W_{\ell T}^{1, p, \boldsymbol{\delta}}\left(T_{u}\right), \\
V_{\Gamma}^{\Sigma} \stackrel{d u}{\longrightarrow} V_{\Gamma}^{T}, \\
L^{p, \boldsymbol{\delta}, d}\left(\overline{\operatorname{End}}_{\mathbb{C}}(T \dot{\Sigma})\right) \stackrel{d u}{\longrightarrow} L^{p, \boldsymbol{\delta}}\left(\overline{\operatorname{Hom}}_{\mathbb{C}}\left(T \dot{\Sigma}, T_{u}\right)\right) .
\end{gathered}
$$

We will stick with this choice of $d$ henceforward.

Using the fact that $z^{k}$ is holomorphic on the punctured disk for any $k \in \mathbb{Z}$, it is easy to show that the natural linear Cauchy-Riemann operator on $\Gamma(T \dot{\Sigma})$ defines a bounded linear map

$$
\boldsymbol{D}_{d}^{\Sigma}: W_{T(\partial \Sigma)}^{1, p, \boldsymbol{\delta}, d}(T \dot{\Sigma}) \oplus V_{\Gamma}^{\Sigma} \rightarrow L^{p, \delta, d}\left(\overline{\operatorname{End}}_{\mathbb{C}}(T \dot{\Sigma})\right) .
$$

The next result then follows from Lemma 3.8 by a density argument.

Lemma 3.14. For any $v \in W_{T(\partial \Sigma)}^{1, p, \delta}(T \dot{\Sigma}) \oplus V_{\Gamma}^{\Sigma}, \boldsymbol{D}_{u}(d u(v))=d u\left(\boldsymbol{D}_{d}^{\Sigma} v\right)$.

Lemma 3.15. The operator

$$
\begin{aligned}
\boldsymbol{L}_{d}: T_{j} \mathcal{T} \oplus\left(W_{T(\partial \Sigma)}^{1, p, \boldsymbol{\delta}, d}(T \dot{\Sigma}) \oplus V_{\Gamma}^{\Sigma}\right) & \rightarrow L^{p, \boldsymbol{\delta}, d}\left(\overline{\operatorname{End}}_{\mathbb{C}}(T \dot{\Sigma})\right) \\
(y, v) & \mapsto j y+\boldsymbol{D}_{d}^{\Sigma} v
\end{aligned}
$$

is surjective and has kernel of dimension

$$
\operatorname{dim} \operatorname{ker}\left(\boldsymbol{L}_{d}\right)=2 Z(d u)+\operatorname{dim} \operatorname{Aut}(\dot{\Sigma}, j) .
$$

Proof. We claim first that the result does not depend on the choice of Teichmüller slice $\mathcal{T}$. Indeed, in light of the splitting $L^{p, \delta}\left(\overline{\operatorname{End}}_{\mathbb{C}}(T \dot{\Sigma})\right)=\operatorname{im}\left(\boldsymbol{D}^{\Sigma}\right) \oplus T_{j} \mathcal{T}$ and the natural inclusion of this space in $L^{p, \delta, d}\left(\overline{\operatorname{End}}_{\mathbb{C}}(T \dot{\Sigma})\right)$, an argument analogous to that in the proof of Lemma 3.11 shows that $\boldsymbol{L}_{d}$ has the same image as its natural extension to $L^{p, \delta}\left(\overline{\operatorname{End}}_{\mathbb{C}}(T \dot{\Sigma})\right) \oplus\left(W_{T(\partial \Sigma)}^{1, p, \boldsymbol{\delta}, d}(T \dot{\Sigma}) \oplus V_{\Gamma}^{\Sigma}\right)$. We are thus free to change $\mathcal{T}$ : in particular, we shall use Lemma 3.4 to assume in the following that all $y \in T_{j} \mathcal{T}$ vanish on some fixed neighborhood of $\operatorname{Crit}(u) \cup \Gamma$.

The subscript $d$ is meant to distinguish $\boldsymbol{L}_{d}$ and $\boldsymbol{D}_{d}^{\Sigma}$ from the operators that appeared in Lemma 3.5; we shall continue to denote the latter simply by

$$
\boldsymbol{L}: T_{j} \mathcal{T} \oplus\left(W_{T(\partial \Sigma)}^{1, p, \boldsymbol{\delta}}(T \dot{\Sigma}) \oplus V_{\Gamma}^{\Sigma}\right) \rightarrow L^{p, \boldsymbol{\delta}}\left(\overline{\operatorname{End}}_{\mathbb{C}}(T \dot{\Sigma})\right),
$$

with $\boldsymbol{D}^{\Sigma}$ denoting the restriction to $W_{T(\partial \Sigma)}^{1, p, \delta}(T \dot{\Sigma}) \oplus V_{\Gamma}^{\Sigma}$. The latter has index 
$\operatorname{ind}\left(\boldsymbol{D}^{\Sigma}\right)=\operatorname{dim} \operatorname{Aut}(\dot{\Sigma}, j)-\operatorname{dim} \mathcal{\mathcal { T }}$, whereas Lemma 3.14 implies that $\boldsymbol{D}_{d}^{\Sigma}$ is conjugate to $D_{u}^{T}$, hence

$$
\begin{aligned}
\operatorname{ind}\left(\boldsymbol{D}_{d}^{\Sigma}\right) & =\operatorname{ind}\left(\boldsymbol{D}_{u}^{T}\right)=\operatorname{dim} \operatorname{Aut}(\dot{\Sigma}, j)-\operatorname{dim} \mathcal{T}+2 Z(d u) \\
& =\operatorname{ind}\left(\boldsymbol{D}^{\Sigma}\right)+2 Z(d u)
\end{aligned}
$$

and $\operatorname{ind}\left(\boldsymbol{L}_{d}\right)=\operatorname{ind}(\boldsymbol{L})+2 Z(d u)=2 Z(d u)+\operatorname{dim} \operatorname{Aut}(\dot{\Sigma}, j)$. The result will follow if we can show that $\operatorname{dim} \operatorname{ker}\left(\boldsymbol{L}_{d}\right) \leq \operatorname{ind}(\boldsymbol{L})+2 Z(d u)$.

To this end, define a $2 Z(d u)$-dimensional subspace $P \subset W_{T(\partial \dot{\Sigma})}^{1, p, \delta, d}(T \dot{\Sigma})$ as follows: $P$ shall consist of smooth sections on $\dot{\Sigma} \backslash \operatorname{Crit}(u)$, supported near $\operatorname{Crit}(u)$, which in our chosen holomorphic trivializations near any given $z_{0} \in \operatorname{Crit}(u)$ take the form

$$
\frac{c_{1}}{z}+\frac{c_{2}}{z^{2}}+\cdots+\frac{c_{d\left(z_{0}\right)}}{z^{d\left(z_{0}\right)}}
$$

for $c_{i} \in \mathbb{C}$ if $z_{0} \in$ int $\Sigma$, or $c_{i} \in \mathbb{R}$ if $z_{0} \in \partial \Sigma$. Since every section in $P$ is holomorphic near $\operatorname{Crit}(u)$, there is an obvious extension of $\boldsymbol{L}$,

$$
\boldsymbol{L}^{\prime}: T_{j} \mathcal{T} \oplus\left(W_{T(\partial \Sigma)}^{1, p, \boldsymbol{\delta}}(T \dot{\Sigma}) \oplus V_{\Gamma}^{\Sigma} \oplus P\right) \rightarrow L^{p, \boldsymbol{\delta}}\left(\overline{\operatorname{End}}_{\mathbb{C}}(T \dot{\Sigma})\right),
$$

which has ind $\left(\boldsymbol{L}^{\prime}\right)=\operatorname{ind}(\boldsymbol{L})+\operatorname{dim} P=\operatorname{ind}(\boldsymbol{L})+2 Z(d u)=\operatorname{ind}\left(\boldsymbol{L}_{d}\right)$. Now since $\boldsymbol{L}$ is surjective by Lemma 3.5, so is $\boldsymbol{L}^{\prime}$, and thus $\operatorname{dim} \operatorname{ker}\left(\boldsymbol{L}^{\prime}\right)=\operatorname{ind}(\boldsymbol{L})+2 Z(d u)$.

To finish, we claim that $\operatorname{ker}\left(\boldsymbol{L}_{d}\right) \subset \operatorname{ker}\left(\boldsymbol{L}^{\prime}\right)$. Indeed, suppose $y \in T_{j} \mathcal{T}$ and $v \in W^{1, p, \boldsymbol{\delta}, d}(T \dot{\Sigma}) \oplus V_{\Gamma}^{\Sigma}$ such that

$$
D^{\Sigma} v=-j \circ y .
$$

Then by our assumption on $\mathcal{T}, y$ vanishes near $\operatorname{Crit}(u)$ and $v$ is therefore a holomorphic section in this neighborhood, except possibly at points of Crit $(u)$. Near $z_{0} \in \operatorname{Crit}(u)$, the principal part of $v$ in our holomorphic trivialization must have the form of (3.19): in particular there cannot be an essential singularity or pole of order higher than $d\left(z_{0}\right)$ since $z^{d\left(z_{0}\right)} v(z)$ is of class $W^{1, p}$. There is thus a unique section in $P$ that equals the principal part near $\operatorname{Crit}(u)$, and subtracting this off we obtain a section in $W_{T(\partial \Sigma)}^{1, p, \delta}(T \dot{\Sigma}) \oplus V_{\Gamma}^{\Sigma}$, showing that $v$ belongs to the domain of $\boldsymbol{L}^{\prime}$.

Proof of Theorem 3. Any $v \in T_{u} \mathcal{B}$ can be decomposed uniquely as $v=d u\left(v_{\Sigma}\right)+$ $v_{N}$ where $v_{\Sigma} \in W_{T(\partial \Sigma)}^{1, p, \boldsymbol{\delta}, d}(T \dot{\Sigma}) \oplus V_{\Gamma}^{\Sigma}$ and $v_{N} \in W_{\ell N}^{1, p, \boldsymbol{\delta}}\left(N_{u}\right) \oplus X_{\Gamma}$. Then for $y \in T_{j} \mathcal{T}$, Lemma 3.14 implies

$$
\begin{aligned}
D \bar{\partial}_{J}(j, u)(y, v) & =J \circ T u \circ y+d u\left(\boldsymbol{D}_{d}^{\Sigma} v_{\Sigma}\right)+\boldsymbol{D}_{u}^{N T} v_{N}+\boldsymbol{D}_{u}^{N} v_{N} \\
& =d u\left(j \circ y+\boldsymbol{D}_{d}^{\Sigma} v_{\Sigma}\right)+\boldsymbol{D}_{u}^{N T} v_{N}+\boldsymbol{D}_{u}^{N} v_{N} .
\end{aligned}
$$


The first term is $\boldsymbol{G}_{u} y+\boldsymbol{D}_{u}^{T} d u\left(v_{\Sigma}\right)$, and this maps onto $L^{p, \delta}\left(\overline{\operatorname{Hom}}_{\mathbb{C}}\left(T \dot{\Sigma}, T_{u}\right)\right)$ by Lemma 3.15, with $\operatorname{dim} \operatorname{ker}\left(\boldsymbol{G}_{u}+\boldsymbol{D}_{u_{-}}^{T}\right)=2 Z(d u)+\operatorname{dim} \operatorname{Aut}(\dot{\Sigma}, j)$. The desired description of ker $D \bar{\partial}_{J}(j, u)$ and im $D \bar{\partial}_{J}(j, u)$ now follows easily from this expression since $\boldsymbol{D}_{u}^{N T} v_{N} \in L^{p, \delta}\left(\overline{\operatorname{Hom}}_{\mathbb{C}}\left(T \dot{\Sigma}, T_{u}\right)\right)$.

Example 3.16. Though we have generally assumed $n \geq 2$, Theorem 3 also applies to the case $n=1$ : then the normal bundle has rank zero and $D \bar{\partial}_{J}(j, u)=\boldsymbol{G}_{u}+$ $\boldsymbol{D}_{u}^{T}$, so the theorem says that $D \bar{\partial}_{J}(j, u)$ is a surjective operator of index $2 Z(d u)+$ $\operatorname{dim} \operatorname{Aut}(\dot{\Sigma}, j)$. One can apply this to understand the moduli space $\mathcal{M}\left(\dot{\Sigma}, \dot{\Sigma}^{\prime}\right)$ of asymptotically cylindrical holomorphic maps

$$
\varphi:(\dot{\Sigma}, j) \rightarrow\left(\dot{\Sigma}^{\prime}, j^{\prime}\right)
$$

between two punctured Riemann surfaces $\Sigma \backslash \Gamma$ and $\Sigma^{\prime} \backslash \Gamma^{\prime}$, up to equivalence by automorphisms on the domain. Such maps are equivalent to holomorphic maps $(\Sigma, j) \rightarrow\left(\Sigma^{\prime}, j^{\prime}\right)$ that send $\Gamma$ to $\Gamma^{\prime}$. Combining Theorem 3 and Theorem 0 , we see that for any $\varphi \in \mathcal{M}\left(\dot{\Sigma}, \dot{\Sigma}^{\prime}\right)$, the connected component $\mathcal{M}_{\varphi}\left(\dot{\Sigma}, \dot{\Sigma}^{\prime}\right)$ containing $\varphi$ is a smooth orbifold with

$$
\operatorname{dim} \mathcal{M}_{\varphi}\left(\dot{\Sigma}, \dot{\Sigma}^{\prime}\right)=2 Z(d \varphi),
$$

where of course the right hand side can be computed from the Riemann-Hurwitz formula. This fact is classical, but it will be useful in the proof of Theorem 2 to view it in our particular analytical setup.

Before restricting to the four-dimensional case, we mention one more simple application of Theorem 3. It gives namely an upper bound on the algebraic number of critical points in terms of the dimension of the kernel. For somewhere injective curves in the generic case this is simply the index, and we obtain:

Corollary 3.17. For generic $J$, all somewhere injective curves $u \in \mathcal{M}$ satisfy

$$
2 Z(d u) \leq \operatorname{ind}(u ; \mathbf{c}) .
$$

So for instance, if $\partial \Sigma=\emptyset$ then somewhere injective curves of index 0 or 1 are necessarily immersed for generic $J$. This is a simple version of the folk theorem that generically, spaces of curves with at least a certain number of critical points have positive codimension.

3.5. The transversality criterion in dimension four. We will now show that Theorem 3 implies Theorem 1 in the case $\operatorname{dim} W=4$. The key is the fact that $N_{u} \rightarrow \dot{\Sigma}$ is now a complex line bundle, so $\boldsymbol{D}_{u}^{N}$ will be subject to the constraints of Proposition 2.2. 
Recall from (1.2) the definition of the normal first Chern number $c_{N}(u ; \mathbf{c})$. An easy exercise combining the index formula with the relations (2.3) between winding numbers and Conley-Zehnder indices yields the following alternative definition, reminiscent of (2.4):

$$
\begin{aligned}
c_{N}(u ; \mathbf{c})=c_{1}^{\Phi} & \left(u^{*} T W\right)-\chi(\dot{\Sigma})+\frac{1}{2} \mu^{\Phi}\left(u^{*} T W, \Lambda\right) \\
& +\sum_{z \in \Gamma^{+}} \alpha_{-}^{\Phi}\left(\gamma_{z}+\mathbf{c}_{z}\right)-\sum_{z \in \Gamma^{-}} \alpha_{+}^{\Phi}\left(\gamma_{z}-\mathbf{c}_{z}\right) .
\end{aligned}
$$

Proposition 3.18. If $u \in \mathcal{M}^{\mathbf{c}}$ is not constant, then

$$
c_{1}\left(N_{u}, \ell^{N}, \boldsymbol{A}_{\Gamma} \pm \mathbf{c}_{\Gamma}\right)=c_{N}(u ; \mathbf{c})-Z(d u)
$$

Proof. Choosing appropriate trivializations $\Phi$, the relation follows by a simple calculation using the definitions (3.20) and

$$
\begin{aligned}
c_{1}\left(N_{u}, \ell^{N}, \boldsymbol{A}_{\Gamma} \pm \mathbf{c}_{\Gamma}\right)=c_{1}^{\Phi}\left(N_{u}\right)+ & \frac{1}{2} \mu^{\Phi}\left(N_{u}, \ell^{N}\right) \\
& +\sum_{z \in \Gamma^{+}} \alpha_{-}^{\Phi}\left(\boldsymbol{A}_{z}+\mathbf{c}_{z}\right)-\sum_{z \in \Gamma^{-}} \alpha_{+}^{\Phi}\left(\boldsymbol{A}_{z}-\mathbf{c}_{z}\right)
\end{aligned}
$$

and plugging in (3.16).

To finish the proof of Theorem 1, we relate $\boldsymbol{D}_{u}^{N}$ to a similar operator on a larger weighted domain: for $z \in \Gamma$, regard the numbers $\mathbf{c}_{z}= \pm \delta$ now as exponential weights and, recalling the notation for weighted Sobolev spaces from $\S 2$, extend $\boldsymbol{D}_{u}^{N}$ to a new operator

$$
\tilde{\boldsymbol{D}}_{u}^{N}: W^{1, p, \mathbf{c}_{\Gamma}}\left(N_{u}\right) \rightarrow L^{p, \mathbf{c}_{\Gamma}}\left(\overline{\operatorname{Hom}}_{\mathbb{C}}\left(T \dot{\Sigma}, N_{u}\right)\right) .
$$

The extended operator is conjugate to an operator on non-weighted spaces asymptotic to $\boldsymbol{A}_{\Gamma} \pm \mathbf{c}_{\Gamma}$, so (2.1) gives ind $\left(\widetilde{\boldsymbol{D}}_{u}^{N}\right)=\operatorname{ind}\left(\boldsymbol{D}_{u}^{N}\right)$. Moreover, Proposition 2.2 together with Proposition 3.18 above implies for $\widetilde{\boldsymbol{D}}_{u}^{N}$ precisely the transversality criterion and kernel bound that we would desire for $D_{u}^{N}$. The result then follows because the domain of $\widetilde{\boldsymbol{D}}_{u}^{N}$ contains that of $\boldsymbol{D}_{u}^{N}$, hence $\operatorname{ker} \boldsymbol{D}_{u}^{N} \subset \operatorname{ker} \widetilde{\boldsymbol{D}}_{u}^{N}$.

\section{Application to spaces of embedded curves}

As an application of the transversality theory, we shall in this section state and prove a stronger version of Theorem 2. For preparation, we review in $\$ 4.1$ some basic facts from the intersection theory of asymptotically cylindrical holomorphic curves 
in four dimensions. This theory has been developed by R. Siefring [Sie08] for curves with fixed asymptotic orbits, and is generalized to the Morse-Bott case in [SW]. We expand on this in $\S 4.2$ by proving some useful formulas involving the intersection theory for multiple covers of orbits and holomorphic curves. The proof of Theorem 2 then appears in $\S 4.3$.

For the remainder of this paper we consider only pseudoholomorphic curves without boundary.

Notation. In the following, we will often abbreviate the notation by printing summations with \pm -signs in their index sets, e.g.

$$
\sum_{\left(z_{1}, z_{2}\right) \in \Gamma_{1}^{ \pm} \times \Gamma_{2}^{ \pm}} F_{ \pm}\left(z_{1}, z_{2}\right) .
$$

The intended meaning is then literally,

$$
\sum_{\left(z_{1}, z_{2}\right) \in \Gamma_{1}^{+} \times \Gamma_{2}^{+}} F_{+}\left(z_{1}, z_{2}\right)+\sum_{\left(z_{1}, z_{2}\right) \in \Gamma_{1}^{-} \times \Gamma_{2}^{-}} F_{-}\left(z_{1}, z_{2}\right) .
$$

Several variations on this scheme will appear.

4.1. Intersection theory for punctured holomorphic curves. This section will consist only of definitions and statements; we refer to [Sie08], [SW] for all proofs.

Throughout the following, $(W, J)$ is a 4-dimensional almost complex manifold with cylindrical ends $\left(M_{ \pm}, \mathscr{H}_{ \pm}\right)$, whose vector fields $X_{ \pm}$are Morse-Bott. Suppose $u: \dot{\Sigma} \rightarrow W$ and $u^{\prime}: \dot{\Sigma}^{\prime} \rightarrow W$ are asymptotically cylindrical holomorphic curves belonging to moduli spaces $\mathcal{M}^{\mathbf{c}}$ and $\mathcal{M}^{\mathbf{c}^{\prime}}$ respectively for some choices $\mathbf{c}, \mathbf{c}^{\prime}$ of asymptotic constraints. One of the goals of the intersection theory is to define an integer $i\left(u ; \mathbf{c} \mid u^{\prime} ; \mathbf{c}^{\prime}\right)$ that is invariant as $u$ and $u^{\prime}$ move continuously through $\mathcal{M}^{\mathbf{c}}$ and $\mathcal{M}^{\mathbf{c}^{\prime}}$ respectively, and can be interpreted as an algebraic intersection count for the two curves. One can show (see [Sie08]) that if $u$ and $u^{\prime}$ are geometrically distinct, meaning they do not both cover the same somewhere injective curve, then their intersections occur only within some compact subset, so the algebraic intersection count $u \bullet u^{\prime}$ is indeed finite and nonnegative. It is not however homotopy invariant in general, as intersections can run out to infinity under homotopies. There is nonetheless a well defined notion of an asymptotic intersection number

$$
i_{\infty}\left(u ; \mathbf{c} \mid u^{\prime} ; \mathbf{c}^{\prime}\right) \in \mathbb{Z}
$$

which is also nonnegative, such that the sum

$$
i\left(u ; \mathbf{c} \mid u^{\prime} ; \mathbf{c}^{\prime}\right):=u \cdot u^{\prime}+i_{\infty}\left(u ; \mathbf{c} \mid u^{\prime} ; \mathbf{c}^{\prime}\right)
$$


depends only on the respective connected components $\mathcal{M}_{u}^{\mathbf{c}}$ and $\mathcal{M}_{u^{\prime}}^{\mathbf{c}^{\prime}}$. With some additional effort and (as yet unpublished) analysis, one can show that $i_{\infty}\left(u ; \mathbf{c} \mid u^{\prime} ; \mathbf{c}^{\prime}\right)=0$ for generic somewhere injective curves and generic $J$ : more precisely, the spaces of curves for which $i_{\infty}\left(u ; \mathbf{c} \mid u^{\prime} ; \mathbf{c}^{\prime}\right)>0$ have positive codimension, and so $u \bullet u^{\prime}$ attains the maximal possible value $i\left(u ; \mathbf{c} \mid u^{\prime} ; \mathbf{c}^{\prime}\right)$ generically.

It is useful to phrase the definition of $i\left(u ; \mathbf{c} \mid u^{\prime} ; \mathbf{c}^{\prime}\right)$ in terms of the relative intersection number $u \bullet_{\Phi} u^{\prime}$, where $\Phi$ is an arbitrary choice of trivialization for $\xi_{ \pm}$ along the asymptotic orbits of $u$ and $u^{\prime}$. One computes $u \bullet_{\Phi} u^{\prime}$ by counting the intersections of $u^{\prime}$ with a small perturbation of $u$ that is offset in the $\Phi$-direction at infinity: the resulting integer is homotopy invariant and depends on $\Phi$ up to homotopy. Then as shown in [Sie08], [SW], for each pair of orbits $\gamma, \gamma^{\prime}$ and numbers $\boldsymbol{\epsilon}, \boldsymbol{\epsilon}^{\prime} \in \mathbb{R}$, there are integers $\Omega_{ \pm}^{\Phi}\left(\gamma+\boldsymbol{\epsilon}, \gamma^{\prime}+\boldsymbol{\epsilon}^{\prime}\right)$ such that

$$
i\left(u ; \mathbf{c} \mid u^{\prime} ; \mathbf{c}^{\prime}\right)=u \bullet \Phi u^{\prime}-\sum_{\left(z, z^{\prime}\right) \in \Gamma^{ \pm} \times\left(\Gamma^{\prime}\right)^{ \pm}} \Omega_{ \pm}^{\Phi}\left(\gamma_{z} \pm \mathbf{c}_{z}, \gamma_{z^{\prime}} \pm \mathbf{c}_{z^{\prime}}^{\prime}\right),
$$

with the dependence on $\Phi$ canceling out on the right hand side. The actual definitions of $\Omega_{ \pm}^{\Phi}\left(\gamma+\boldsymbol{\epsilon}, \gamma^{\prime}+\boldsymbol{\epsilon}^{\prime}\right)$ are as follows. We set $\Omega_{ \pm}^{\Phi}\left(\gamma+\boldsymbol{\epsilon}, \gamma^{\prime}+\boldsymbol{\epsilon}^{\prime}\right)=0$ if $\gamma$ and $\gamma^{\prime}$ are geometrically distinct orbits, and for any simply covered orbit $\gamma$ and $m, n \in \mathbb{N}$, if $\gamma^{m}$ and $\gamma^{n}$ denote the corresponding covers of $\gamma$, let

$$
\Omega_{ \pm}^{\Phi}\left(\gamma^{m}+\boldsymbol{\epsilon}, \gamma^{n}+\boldsymbol{\epsilon}^{\prime}\right)=m n \cdot \min \left\{\frac{\mp \alpha_{\mp}^{\Phi}\left(\gamma^{m}+\boldsymbol{\epsilon}\right)}{m}, \frac{\mp \alpha_{\mp}^{\Phi}\left(\gamma^{n}+\boldsymbol{\epsilon}^{\prime}\right)}{n}\right\} .
$$

We shall use the abbreviated notation $\Omega_{ \pm}^{\Phi}\left(\gamma, \gamma^{\prime}\right)$ when $\boldsymbol{\epsilon}=\boldsymbol{\epsilon}^{\prime}=0$. Observe that the right hand side of (4.2) makes sense even when $u$ and $u^{\prime}$ are not geometrically distinct; in particular, we can use it to define $i(u ; \mathbf{c} \mid u$; $)$, which is the appropriate generalization of a "self-intersection number" for punctured holomorphic curves.

If $u$ and $u^{\prime}$ are geometrically distinct, then we can directly define the asymptotic contribution $i_{\infty}\left(u ; \mathbf{c} \mid u^{\prime} ; \mathbf{c}^{\prime}\right)$, thus giving a more conceptually revealing definition of $i\left(u ; \mathbf{c} \mid u^{\prime} ; \mathbf{c}^{\prime}\right)$ via (4.1). Indeed, any pair of punctures for $u$ and $u^{\prime}$ that have the same sign and indistinct orbits offers a potential for intersections to be "hidden at infinity". For two such punctures $z \in \Gamma^{ \pm}$and $z^{\prime} \in\left(\Gamma^{\prime}\right)^{ \pm}$, denote by

$$
i_{\infty}^{\Phi}\left(u_{z}, u_{z^{\prime}}^{\prime}\right)
$$

the relative asymptotic intersection: this is computed by restricting both curves to suitably small cylindrical neighborhoods of the respective punctures and counting any intersections that appear near infinity after perturbing $u$ in the $\Phi$-direction. It turns out that whenever both curves are $J$-holomorphic, $i_{\infty}^{\Phi}\left(u_{z}, u_{z^{\prime}}^{\prime}\right)$ is a priori bounded from below by $\Omega_{ \pm}^{\Phi}\left(\gamma_{z}, \gamma_{z^{\prime}}\right)$ : thus the integer

$$
i_{\infty}\left(u_{z}, u_{z^{\prime}}^{\prime}\right):=i_{\infty}^{\Phi}\left(u_{z}, u_{z^{\prime}}^{\prime}\right)-\Omega_{ \pm}^{\Phi}\left(\gamma_{z}, \gamma_{z^{\prime}}\right)
$$


is nonnegative and independent of $\Phi$. Intuitively, it counts the potential intersections of these two ends that can "emerge from infinity" under homotopies of $u$ and $u^{\prime}$ that fix their asymptotic orbits. Additional intersections may appear if either orbit is unconstrained and allowed to move in a Morse-Bott family: the number of these is also nonnegative and turns out to depend only on the orbits and their constraints. Thus for any orbits $\gamma, \gamma^{\prime}$ and numbers $\boldsymbol{\epsilon}, \boldsymbol{\epsilon}^{\prime} \in \mathbb{R}$, define

$$
i_{\mathrm{MB}}^{ \pm}\left(\gamma+\boldsymbol{\epsilon}, \gamma^{\prime}+\boldsymbol{\epsilon}^{\prime}\right)=\Omega_{ \pm}^{\Phi}\left(\gamma, \gamma^{\prime}\right)-\Omega_{ \pm}^{\Phi}\left(\gamma+\boldsymbol{\epsilon}, \gamma^{\prime}+\boldsymbol{\epsilon}^{\prime}\right) .
$$

We will interpret $i_{\mathrm{MB}}^{ \pm}\left(\gamma_{z} \pm \mathbf{c}_{z}, \gamma_{z^{\prime}} \pm \mathbf{c}_{z^{\prime}}^{\prime}\right)$ as the number of "extra" hidden intersections not counted by $i_{\infty}\left(u_{z}, u_{z^{\prime}}^{\prime}\right)$ that can emerge as these two ends move generically according to their respective constraints, potentially shifting the asymptotic orbits. The total asymptotic intersection number is then

$$
i_{\infty}\left(u ; \mathbf{c} \mid u^{\prime} ; \mathbf{c}^{\prime}\right):=\sum_{\left(z, z^{\prime}\right) \in \Gamma^{ \pm} \times\left(\Gamma^{\prime}\right)^{ \pm}}\left[i_{\infty}\left(u_{z}, u_{z^{\prime}}^{\prime}\right)+i_{\mathrm{MB}}^{ \pm}\left(\gamma_{z} \pm \mathbf{c}_{z}, \gamma_{z^{\prime}} \pm \mathbf{c}_{z^{\prime}}^{\prime}\right)\right] .
$$

Each individual term in this sum is nonnegative, and can be expected to vanish under generic perturbations of $u$ and $u^{\prime}$ as "potential" intersections become real.

If $u: \dot{\Sigma} \rightarrow W$ is somewhere injective, we recall from [MW95] that $u$ admits a local description near any critical point allowing one to define a nonnegative singularity index $\delta(u)$ : it gives an algebraic count of self-intersections $u(z)=u\left(z^{\prime}\right)$ for $z \neq z^{\prime}$ after making local perturbations so that $u$ becomes immersed. As shown in [Sie08], this still makes sense in the punctured case because $u$ is necessarily embedded outside of some compact subset: then $\delta(u) \geq 0$, with equality if and only if $u$ is embedded. It is however possible for self-intersections to escape to infinity under homotopies, thus $\delta(u)$ is not homotopy invariant, but as with the intersection number, one can add a nonnegative asymptotic singularity index $\delta_{\infty}(u ; \mathbf{c})$ so that the sum

$$
\operatorname{sing}(u ; \mathbf{c}):=\delta(u)+\delta_{\infty}(u ; \mathbf{c})
$$

depends only on the connected component $\mathcal{M}_{u}^{\mathbf{c}}$, and equals $\delta(u)$ generically but not always. The condition $\operatorname{sing}(u ; \mathbf{c})=0$ is then necessary and sufficient so that all somewhere injective curves in $\mathcal{M}_{u}^{\mathbf{c}}$ should be embedded for generic $J$; note that one still may have $u$ embedded if $\operatorname{sing}(u ; \mathbf{c})>0$, but then generic curves close to $u$ will not be. The asymptotic contribution is a sum of the form

$$
\begin{aligned}
2 \delta_{\infty}(u ; \mathbf{c})= & \sum_{z \neq z^{\prime} \in \Gamma^{ \pm}}\left[i_{\infty}\left(u_{z}, u_{z^{\prime}}\right)+i_{\mathrm{MB}}^{ \pm}\left(\gamma_{z} \pm \mathbf{c}_{z}, \gamma_{z^{\prime}} \pm \mathbf{c}_{z^{\prime}}\right)\right] \\
& +\sum_{z \in \Gamma^{ \pm}}\left[2 \delta_{\infty}\left(u_{z}\right)+2 \delta_{\mathrm{MB}}^{ \pm}\left(\gamma_{z} \pm \mathbf{c}_{z}\right)\right],
\end{aligned}
$$

in which every term is nonnegative if $u$ is $J$-holomorphic. We interpret $\delta_{\infty}\left(u_{z}\right)$ as the number of self-intersections near the puncture $z$ that may emerge from infinity 
under generic homotopies fixing the orbit $\gamma_{z}$; this can happen if $\gamma_{z}$ is multiply covered, as distinct branches of the cylinder approaching $\gamma_{z}$ run into each other under perturbation. Define

$$
2 \delta_{\infty}\left(u_{z}\right)=i_{\infty}^{\Phi}\left(u_{z}, u_{z}\right)-\Omega_{ \pm}^{\Phi}\left(\gamma_{z}\right),
$$

where $\Omega_{ \pm}^{\Phi}(\gamma) \in \mathbb{Z}$ is the "self-intersection analogue" of $\Omega_{ \pm}^{\Phi}\left(\gamma, \gamma^{\prime}\right)$, giving a different theoretical minimum for $i_{\infty}^{\Phi}\left(u_{z}, u_{z}\right)$ since the two ends are identical. To write it down explicitly for the $k$-fold cover of a simple orbit $\gamma$, choose any nontrivial eigenfunction $e_{\mp}$ of $\boldsymbol{A}_{\gamma^{k}}$ whose winding about $\gamma^{k}$ equals $\alpha_{\mp}^{\Phi}\left(\gamma^{k}\right)$, and note that its covering number $\operatorname{cov}\left(e_{\mp}\right) \in \mathbb{N}$ depends on $k$ and $\alpha_{\mp}^{\Phi}\left(\gamma^{k}\right)$ but not on the choice $e_{\mp}$ (cf. Lemma 4.2). Thus we denote

$$
\operatorname{cov} \mp\left(\gamma^{k}\right):=\operatorname{cov}\left(e_{\mp}\right),
$$

and then define

$$
\Omega_{ \pm}^{\Phi}\left(\gamma^{k}\right)=\mp(k-1) \alpha_{\mp}^{\Phi}\left(\gamma^{k}\right)+\left[\operatorname{cov}_{\mp}\left(\gamma^{k}\right)-1\right] .
$$

Similarly, $\delta_{\mathrm{MB}}^{ \pm}\left(\gamma_{z} \pm \mathbf{c}_{z}\right)$ counts further self-intersections that may emerge if $\gamma_{z}$ is allowed to move in a Morse-Bott family. This does not happen if every orbit in the family has the same minimal period, but if $\gamma_{z}$ converges to an orbit with smaller minimal period (and thus higher covering number), the existence of additional branches can hide extra intersections at infinity. The following characterization of Morse-Bott manifolds will be useful.

Proposition 4.1. If $M$ is a 3-manifold with a Morse-Bott vector field $X$, then every Morse-Bott submanifold $P \subset M$ can be described as follows. There exists a number $\tau>0$ such that all but a discrete set of orbits in $P$ have minimal period $\tau$; we shall call these generic orbits. The other orbits will be called exceptional: any such orbit with period $\tau$ is an $m$-fold cover of a simply covered orbit $\gamma$ for some $m \geq 2$ (called the isotropy), and $\gamma^{k}$ is nondegenerate for all $k \in\{1, \ldots, m-1\}$. The isotropy of an exceptional orbit is always 2 if $\operatorname{dim} P=2$.

Now, define $\delta_{\mathrm{MB}}^{ \pm}(\gamma \pm \delta)=0$ if $\boldsymbol{\delta}>0$; recall this case is associated with a constraint that fixes $\gamma_{z}$, thus there can be no "extra" self-intersections appearing due to Morse-Bott considerations. The definition is as follows if $\delta<0$ : given an orbit $\gamma$, set $\gamma_{\epsilon}=\gamma$ if it is nondegenerate, otherwise let $\gamma_{\epsilon}$ denote any nearby generic orbit in the same Morse-Bott family as $\gamma$. If $\gamma_{\epsilon}$ is simply covered, $k \in \mathbb{N}$, and $\gamma$ has isotropy $m \in \mathbb{N}$, then set

$$
2 \delta_{\mathrm{MB}}^{ \pm}\left(\gamma^{k} \pm \boldsymbol{\delta}\right)=k(m-1) \nu_{\mp}\left(\gamma^{k}\right)+\operatorname{cov}_{\mp}\left(\gamma^{k}\right)-\operatorname{cov}_{\mp}\left(\gamma_{\epsilon}^{k}\right),
$$

where $\nu_{\mp}(\gamma) \in\{0,1\}$ is defined in (3.4). Observe that the two covering terms refer to homotopic eigenfunctions $e$ of $\boldsymbol{A}_{\gamma^{k}}$ and $e_{\epsilon}$ of $\boldsymbol{A}_{\gamma_{\epsilon}^{k}}$, so if $e_{\epsilon}$ is an $n$-fold cover then 
$e$ is as well, hence $\operatorname{cov}_{\mp}\left(\gamma^{k}\right) \geq \operatorname{cov}_{\mp}\left(\gamma_{\epsilon}^{k}\right)$. The inequality may sometimes be strict, because $e$ is attached to a $k m$-covered orbit, while the orbit of $e_{\epsilon}$ is only $k$-covered. In any case, clearly $\delta_{\mathrm{MB}}^{ \pm}\left(\gamma^{k} \pm \delta\right) \geq 0$ in general, and it vanishes whenever $\gamma$ is a generic orbit.

For the curve $u \in \mathcal{M}^{\mathbf{c}}$, choose for each $z \in \Gamma$ a generic perturbation $\gamma_{z}^{\epsilon}$ of the orbit $\gamma_{z}$, setting $\gamma_{z}^{\epsilon}=\gamma_{z}$ if either $\gamma_{z}$ is nondegenerate or $z \in \Gamma_{C}$. Then let

$$
\operatorname{cov}_{\infty}\left(\gamma_{\Gamma} ; \mathbf{c}\right)=\sum_{z \in \Gamma^{ \pm}}\left[\operatorname{cov}_{\mp}\left(\gamma_{z}^{\epsilon}\right)-1\right]
$$

and

$$
\operatorname{cov}_{\mathrm{MB}}\left(\gamma_{\Gamma} ; \mathbf{c}\right)=\sum_{z \in \Gamma_{U}^{ \pm}}\left[\operatorname{cov}\left(\gamma_{z}^{\epsilon}\right)-1\right] \cdot v_{\mp}\left(\gamma_{z}\right),
$$

with $\operatorname{cov}\left(\gamma_{z}^{\epsilon}\right)$ denoting the covering number of $\gamma_{z}^{\epsilon}$.

Theorem (Adjunction formula [Sie08], [SW]). For any somewhere injective curve $u \in \mathcal{M}^{\mathbf{c}}$,

$$
i(u ; \mathbf{c} \mid u ; \mathbf{c})=2 \operatorname{sing}(u ; \mathbf{c})+c_{N}(u ; \mathbf{c})+\operatorname{cov}_{\infty}\left(\gamma_{\Gamma} ; \mathbf{c}\right)+\operatorname{cov}_{M B}\left(\gamma_{\Gamma} ; \mathbf{c}\right) .
$$

4.2. Some covering relations. It will be useful to have formulas relating the intersection invariants of holomorphic curves and their multiple covers. A prerequisite for this is to have corresponding covering formulas for periodic orbits, so to start with, assume $M$ is a 3-manifold with stable Hamiltonian structure $\mathscr{H}=(X, \xi, \omega, J)$. Given an orbit $\gamma$, we shall denote the corresponding asymptotic operator by $\boldsymbol{A}_{\gamma}$ and the $k$-fold cover of $\gamma$ by $\gamma^{k}$. Then if $\boldsymbol{A}_{\gamma} e=\lambda e$, the eigenfunction has a $k$-fold cover $e^{k}$ such that $\boldsymbol{A}_{\gamma^{k}} e^{k}=k \lambda e^{k}$. In general, we say that an eigenfunction $f$ of $\boldsymbol{A}_{\gamma^{k}}$ is a $k$-fold cover if there exists an eigenfunction $e$ of $\boldsymbol{A}_{\gamma}$ such that $f=e^{k}$.

In the following, whenever a trivialization $\Phi$ along an orbit $\gamma$ appears, we will use the same notation $\Phi$ to denote the resulting induced trivializations along all covers of $\gamma$.

Lemma 4.2 ([Wena], Lemma 3.5). Suppose $\Phi$ is a trivialization along $\gamma$. Then a nontrivial eigenfunction e of $\boldsymbol{A}_{\gamma^{k}}$ is a $k$-fold cover if and only if $\operatorname{wind}^{\Phi}(e) \in k \mathbb{Z}$.

Lemma 4.3. Suppose $\gamma$ is a periodic orbit of $X$ and $\boldsymbol{\epsilon} \in \mathbb{R}$. If $\boldsymbol{A}_{\gamma}+\boldsymbol{\epsilon}$ is nondegenerate and $p(\gamma+\boldsymbol{\epsilon})=0$, then $\boldsymbol{A}_{\gamma^{k}}+k \boldsymbol{\epsilon}$ is nondegenerate and $p\left(\gamma^{k}+k \boldsymbol{\epsilon}\right)=0$ for all $k \in \mathbb{N}$.

Proof. If $p(\gamma+\boldsymbol{\epsilon})=0$, then $\sigma\left(\boldsymbol{A}_{\gamma}+\boldsymbol{\epsilon}\right)$ contains a pair of neighboring eigenvalues with opposite signs and eigenfunctions of the same winding number. The $k$-fold covers of these are eigenfunctions of $\boldsymbol{A}_{\gamma^{k}}+k \boldsymbol{\epsilon}$ with the same properties, thus $\boldsymbol{A}_{\gamma^{k}}+k \boldsymbol{\epsilon}$ is nondegenerate and has even parity. 
Remark 4.4. If $\epsilon \in \mathbb{R}$ is sufficiently close but not equal to zero, then we may always assume that for all $k \in \mathbb{N}$ up to some arbitrarily large (but finite) bound, $\boldsymbol{A}_{\gamma^{k}}+\boldsymbol{\epsilon}$ is nondegenerate and $\alpha_{ \pm}^{\Phi}\left(\gamma^{k}+k \boldsymbol{\epsilon}\right)=\alpha_{ \pm}^{\Phi}\left(\gamma^{k}+\boldsymbol{\epsilon}\right)$. We can thus replace $k \boldsymbol{\epsilon}$ with $\boldsymbol{\epsilon}$ in the statement above whenever $\epsilon$ is assumed close to zero, and the same applies to several statements below.

Corollary 4.5. For any exceptional orbit in a Morse-Bott family, the underlying simple orbit and all of its nondegenerate covers are odd.

Proposition 4.6. For any periodic orbit $\gamma$ of $X, k \in \mathbb{N}$ and $\epsilon \in \mathbb{R}$, there exist integers $q_{ \pm}(\gamma+\boldsymbol{\epsilon} ; k) \in\{0, \ldots, k-1\}$ such that

$$
\alpha_{ \pm}^{\Phi}\left(\gamma^{k}+k \boldsymbol{\epsilon}\right)=k \alpha_{ \pm}^{\Phi}(\gamma+\boldsymbol{\epsilon}) \mp q_{ \pm}(\gamma+\boldsymbol{\epsilon} ; k) .
$$

Proof. The integer $q_{ \pm}(\gamma+\boldsymbol{\epsilon} ; k):=\mp\left[\alpha_{ \pm}^{\Phi}\left(\gamma^{k}+\boldsymbol{\epsilon}\right)-k \alpha_{ \pm}^{\Phi}(\gamma+\boldsymbol{\epsilon})\right]$ is well defined after observing that all dependence on $\Phi$ in the right hand side cancels, so it remains only to show that this number is between 0 and $k-1$. Consider first the case $\epsilon=0$, and choose a trivialization $\Phi_{0}$ along $\gamma$ such that $\alpha_{-}^{\Phi_{0}}(\gamma)=0$. Then there exists an eigenfunction $e_{-}$of $\boldsymbol{A}_{\gamma}$ with negative eigenvalue and wind ${ }^{\Phi_{0}}\left(e_{-}\right)=0$, and another eigenfunction $e_{+}$with nonnegative eigenvalue and wind ${ }^{\Phi_{0}}\left(e_{+}\right)=1$; moreover there are no eigenfunctions with eigenvalue strictly between that of $e_{-}$and 0 . Moving to the $k$-fold cover, we obtain eigenfunctions $e_{-}^{k}$ and $e_{+}^{k}$ of $\boldsymbol{A}_{\gamma^{k}}$ with

$$
\begin{array}{cl}
\text { wind }^{\Phi_{0}}\left(e_{-}^{k}\right)=0 & \text { eigenvalue }<0, \\
\text { wind }^{\Phi_{0}}\left(e_{+}^{k}\right)=k & \text { eigenvalue } \geq 0,
\end{array}
$$

and there is no $k$-fold covered eigenfunction with eigenvalue strictly between that of $e_{-}^{k}$ and 0 . Then by Lemma 4.2, this range of the spectrum of $\boldsymbol{A}_{\gamma^{k}}$ contains no eigenfunctions with winding $k$. Since the winding depends monotonically on the eigenvalue, this implies $\alpha_{-}^{\Phi_{0}}\left(\gamma^{k}\right) \in\{0, \ldots, k-1\}$. An analogous argument gives the corresponding result for $\alpha_{+}$. Finally if $\epsilon \neq 0$, the arguments above give the same relation between the eigenfunctions of $\boldsymbol{A}_{\gamma}+\boldsymbol{\epsilon}$ and $\boldsymbol{A}_{\gamma^{k}}+k \boldsymbol{\epsilon}$.

In preparation for the next lemma, for any orbit $\gamma$, numbers $m, n, k \in \mathbb{N}$ and $\boldsymbol{\delta}, \boldsymbol{\epsilon} \in \mathbb{R}$, define the nonnegative integers

$$
\begin{array}{r}
\tilde{q}_{ \pm}\left(\gamma^{m}+\boldsymbol{\delta}, \gamma^{n}+\boldsymbol{\epsilon} ; k\right)=k m n \cdot \min \left\{\frac{\mp \alpha_{\mp}^{\Phi}\left(\gamma^{m}+\boldsymbol{\delta}\right)}{m}, \frac{\mp \alpha_{\mp}\left(\gamma^{n}+\boldsymbol{\epsilon}\right)}{n}\right\} \\
-k m n \cdot \min \left\{\frac{\mp \alpha_{\mp}^{\Phi}\left(\gamma^{m}+\boldsymbol{\delta}\right)}{m}-\frac{q \mp\left(\gamma^{m}+\boldsymbol{\delta} ; k\right)}{k m}, \frac{\mp \alpha_{\mp}\left(\gamma^{n}+\boldsymbol{\epsilon}\right)}{n}\right\} .
\end{array}
$$

Then a simple computation using the definitions of $\Omega_{ \pm}^{\Phi}\left(\gamma+\boldsymbol{\epsilon}, \gamma^{\prime}+\boldsymbol{\epsilon}^{\prime}\right)$ and $q_{ \pm}(\gamma+\boldsymbol{\epsilon} ; k)$ implies: 
Lemma 4.7. For any simply covered orbit $\gamma, m, n, k \in \mathbb{N}$ and $\boldsymbol{\delta}, \boldsymbol{\epsilon} \in \mathbb{R}$,

$$
\Omega_{ \pm}^{\Phi}\left(\gamma^{k m}+k \boldsymbol{\delta}, \gamma^{n}+\boldsymbol{\epsilon}\right)=k \cdot \Omega_{ \pm}^{\Phi}\left(\gamma^{m}+\boldsymbol{\delta}, \gamma^{n}+\boldsymbol{\epsilon}\right)-\tilde{q}_{ \pm}\left(\gamma^{m}+\boldsymbol{\delta}, \gamma^{n}+\boldsymbol{\epsilon} ; k\right) .
$$

Returning now to the context of a 4-manifold $W$ with Morse-Bott cylindrical ends $\left(M_{ \pm}, \mathscr{H}_{ \pm}\right)$, let us fix the following notation: $u \in \mathcal{M}^{\mathbf{c}}$ is a holomorphic curve with domain $(\Sigma \backslash \Gamma, j),(\Sigma, j)$ and $(\widetilde{\Sigma}, \tilde{J})$ are closed Riemann surfaces, and $\varphi:(\widetilde{\Sigma}, \tilde{J}) \rightarrow$ $(\Sigma, j)$ is a holomorphic branched cover of degree $\operatorname{deg}(\varphi) \in \mathbb{N}$. This restricts to a branched cover of punctured surfaces

$$
\dot{\varphi}: \tilde{\Sigma} \backslash \widetilde{\Gamma} \rightarrow \Sigma \backslash \Gamma,
$$

where $\widetilde{\Gamma}:=\varphi^{-1}(\Gamma)$, and there is a resulting holomorphic curve $u \circ \varphi: \widetilde{\Sigma} \backslash \widetilde{\Gamma} \rightarrow W$. Its asymptotic orbits are related to those of $u$ by

$$
\gamma_{z}=\gamma_{\varphi(z)}^{k_{z}}
$$

at each $z \in \widetilde{\Gamma}$, where $k_{z}:=\operatorname{ord}(d \varphi ; z)+1$, so that $\varphi$ is $k_{z}$-to- 1 near $z$. The constraints $\mathbf{c}$ on $\Gamma$ can then be pulled back to constraints $\varphi^{*} \mathbf{c}$ on $\widetilde{\Gamma}$ like so: for any $\zeta \in \Gamma$ constrained to the orbit $\gamma_{\zeta}$, define $\varphi^{*} \mathbf{c}$ by fixing the orbit $\gamma_{\zeta}^{k_{z}}$ at each $z \in \varphi^{-1}(\zeta)$. Then $u \circ \varphi \in \mathcal{M}^{\varphi^{*} \mathbf{c}}$.

Proposition 4.8. For $u \in \mathcal{M}^{\mathbf{c}}$ and the cover $u \circ \varphi \in \mathcal{M}^{\varphi^{*} \mathbf{c}}$ defined above,

$$
c_{N}\left(u \circ \varphi ; \varphi^{*} \mathbf{c}\right)=\operatorname{deg}(\varphi) \cdot c_{N}(u ; \mathbf{c})+Z(d \dot{\varphi})+Q,
$$

where $Q$ is a nonnegative integer. Specifically,

$$
Q=\sum_{z \in \tilde{\Gamma}^{ \pm}} q_{\mp}\left(\gamma_{\varphi(z)} \pm \mathbf{c}_{\varphi(z)} ; k_{z}\right) .
$$

Proof. Denote $\tilde{u}:=u \circ \varphi, \tilde{\mathbf{c}}:=\varphi^{*} \mathbf{c}$ and observe that for each $\zeta \in \Gamma, \sum_{z \in \varphi^{-1}(\zeta)} k_{z}=$ $k:=\operatorname{deg}(\varphi)$. Note also that by extending $\dot{\varphi}$ to the circle compactifications of $\widetilde{\Sigma} \backslash \widetilde{\Gamma}$ and $\Sigma \backslash \Gamma$, one can apply the Riemann-Hurwitz formula and obtain

$$
Z(d \dot{\varphi})=-\chi(\tilde{\Sigma} \backslash \tilde{\Gamma})+k \chi(\Sigma \backslash \Gamma) .
$$

Then using (4.9) and Remark 4.4,

$$
\begin{aligned}
c_{N}(\tilde{u} ; \tilde{\mathbf{c}})= & c_{1}^{\Phi}\left(\varphi^{*} u^{*} T W\right)-\chi(\tilde{\Sigma} \backslash \tilde{\Gamma})+\sum_{\zeta \in \Gamma^{ \pm}} \sum_{z \in \varphi^{-1}(\zeta)} \pm \alpha_{\mp}^{\Phi}\left(\gamma_{\zeta}^{k_{z}} \pm \tilde{\mathbf{c}}_{z}\right) \\
= & k c_{1}^{\Phi}\left(u^{*} T W\right)-k \chi(\Sigma \backslash \Gamma)+Z(d \dot{\varphi})+k \sum_{\zeta \in \Gamma^{ \pm}} \pm \alpha_{\mp}^{\Phi}\left(\gamma_{\zeta} \pm \mathbf{c}_{\zeta}\right) \\
& +\sum_{\zeta \in \Gamma^{ \pm}} \sum_{z \in \varphi^{-1}(\zeta)} \pm\left(\alpha_{\mp}^{\Phi}\left(\gamma_{\zeta}^{k_{z}} \pm \tilde{\mathbf{c}}_{z}\right)-k_{z} \alpha_{\mp}^{\Phi}\left(\gamma_{\zeta} \pm \mathbf{c}_{\zeta}\right)\right)
\end{aligned}
$$




$$
=k \cdot c_{N}(u ; \mathbf{c})+Z(d \dot{\varphi})+\sum_{z \in \widetilde{\Gamma}^{ \pm}} q_{\mp}\left(\gamma_{\varphi(z)} \pm \mathbf{c}_{\varphi(z)} ; k_{z}\right) .
$$

Proposition 4.9. For the cover $u \circ \varphi \in \mathcal{M}^{\varphi^{*} \mathbf{c}}$ as in Proposition 4.8 and any other curve $v \in \mathcal{M}^{\mathbf{c}^{\prime}}$,

$$
i\left(u \circ \varphi ; \varphi^{*} \mathbf{c} \mid v ; \mathbf{c}^{\prime}\right) \geq \operatorname{deg}(\varphi) \cdot i\left(u ; \mathbf{c} \mid v ; \mathbf{c}^{\prime}\right) .
$$

Proof. Again denote $k:=\operatorname{deg}(\varphi), k_{z}:=\operatorname{ord}(d \varphi ; z)+1 \in \mathbb{N}$ for each $z \in \widetilde{\Gamma}, \tilde{u}:=$ $u \circ \varphi$ and $\tilde{\mathbf{c}}:=\varphi^{*} \mathbf{c}$. The relative intersection number satisfies $\tilde{u} \bullet_{\Phi} v=k\left(u \bullet_{\Phi} v\right)$. Writing the puncture set of $v$ as $\Gamma^{\prime}$, we apply Lemma 4.7 with Remark 4.4 in mind and find

$$
\begin{aligned}
& i\left(\tilde{u} ; \tilde{\mathbf{c}} \mid v ; \mathbf{c}^{\prime}\right)=\tilde{u} \bullet \Phi v-\sum_{\left(z, z^{\prime}\right) \in \tilde{\Gamma}^{ \pm} \times\left(\Gamma^{\prime}\right)^{ \pm}} \Omega_{ \pm}^{\Phi}\left(\gamma_{z} \pm \tilde{\mathbf{c}}_{z}, \gamma_{z^{\prime}} \pm \mathbf{c}_{z^{\prime}}^{\prime}\right) \\
& =k \cdot(u \cdot \Phi v)-\sum_{\left(\zeta, z^{\prime}\right) \in \Gamma^{ \pm} \times\left(\Gamma^{\prime}\right)^{ \pm}}\left(\sum_{z \in \varphi^{-1}(\zeta)} \Omega_{ \pm}^{\Phi}\left(\gamma_{\zeta}^{k_{z}} \pm \tilde{\mathbf{c}}_{z}, \gamma_{z^{\prime}} \pm \mathbf{c}_{z^{\prime}}^{\prime}\right)\right) \\
& =k \cdot\left(u \bullet_{\Phi} v\right)-\sum_{\left(\zeta, z^{\prime}\right) \in \Gamma^{ \pm} \times\left(\Gamma^{\prime}\right)^{ \pm}}\left(\sum _ { z \in \varphi ^ { - 1 } ( \zeta ) } \left[k_{z} \Omega_{ \pm}^{\Phi}\left(\gamma_{\zeta} \pm \mathbf{c}_{\zeta}, \gamma_{z^{\prime}} \pm \mathbf{c}_{z^{\prime}}^{\prime}\right)\right.\right. \\
& \left.\left.-\tilde{q}_{ \pm}\left(\gamma_{\zeta} \pm \mathbf{c}_{\zeta}, \gamma_{z^{\prime}} \pm \mathbf{c}_{z^{\prime}}^{\prime} ; k_{z}\right)\right]\right) \\
& =k \cdot(u \cdot \Phi v)-k \sum_{\left(\zeta, z^{\prime}\right) \in \Gamma^{ \pm} \times\left(\Gamma^{\prime}\right)^{ \pm}} \Omega_{ \pm}^{\Phi}\left(\gamma_{\zeta} \pm \mathbf{c}_{\zeta} \mid \gamma_{z^{\prime}} \pm \mathbf{c}_{z^{\prime}}^{\prime}\right) \\
& +\sum_{\left(z, z^{\prime}\right) \in \widetilde{\Gamma}^{ \pm} \times\left(\Gamma^{\prime}\right)^{ \pm}} \tilde{q}_{ \pm}\left(\gamma_{\varphi(z)} \pm \mathbf{c}_{\varphi(z)}\left|\gamma_{z^{\prime}} \pm \mathbf{c}_{z^{\prime}}^{\prime}\right| k_{z}\right) \\
& =k \cdot i\left(u ; \mathbf{c} \mid v ; \mathbf{c}^{\prime}\right)+\sum_{\left(z, z^{\prime}\right) \in \widetilde{\Gamma}^{ \pm} \times\left(\Gamma^{\prime}\right)^{ \pm}} \tilde{q}_{ \pm}\left(\gamma_{\varphi(z)} \pm \tilde{\mathbf{c}}_{\varphi(z)}\left|\gamma_{z^{\prime}} \pm \mathbf{c}_{z^{\prime}}^{\prime}\right| k_{z}\right) .
\end{aligned}
$$

The last term is nonnegative.

Definition 4.10. For a given set of punctures $\Gamma$, the set of all choices of asymptotic constraints on $\Gamma$ admits a partial order defined as follows. We say $\mathbf{c}^{-} \leq \mathbf{c}^{+}$if for every $z \in \Gamma$ at which the asymptotic orbit $\gamma_{z}$ is constrained by $\mathbf{c}^{-}$, it is also constrained by $\mathbf{c}^{+}$to the same orbit.

Observe that if $\mathbf{c}^{-} \leq \mathbf{c}+$, then $\mathcal{M}^{\mathbf{c}+} \subset \mathcal{M}^{\mathbf{c}^{-}}$and $\mathbf{c}_{z}^{-} \leq \mathbf{c}_{z}^{+}$for each $z \in \Gamma$. One expects in general that weaker constraints should lead to larger intersection numbers, as intersections can more easily emerge from infinity under more general homotopies. Indeed, using $\mathbf{c}_{z}^{-} \leq \mathbf{c}_{z}^{+}$together with the fact that $\alpha_{\mp}^{\Phi}(\gamma+\boldsymbol{\epsilon})$ always has monotone decreasing dependence on $\boldsymbol{\epsilon}$, we easily derive the following: 
Proposition 4.11. If $\mathbf{c}^{-} \leq \mathbf{c}^{+}$and $u \in \mathcal{M}^{\mathbf{c}^{+}}$, then

$$
c_{N}\left(u ; \mathbf{c}^{-}\right) \geq c_{N}\left(u ; \mathbf{c}^{+}\right) .
$$

Moreover, for any other curve $v \in \mathcal{M}^{\mathbf{c}}$,

$$
i\left(u ; \mathbf{c}^{-} \mid v ; \mathbf{c}\right) \geq i\left(u ; \mathbf{c}^{+} \mid v ; \mathbf{c}\right) .
$$

4.3. Multiply covered limits are immersed. We shall now state and prove a parametrized version of Theorem 2 .

Definition 4.12. We will say that $u \in \mathcal{M}^{\mathbf{c}}$ is a stable, nicely embedded curve (with respect to the constraints $\mathbf{c}$ ) if it is somewhere injective and satisfies the following relations:

(1) $i(u ; \mathbf{c} \mid u ; \mathbf{c}) \leq 0$,

(2) $\operatorname{ind}(u ; \mathbf{c}) \geq 0$,

(3) $\operatorname{ind}(u ; \mathbf{c})>c_{N}(u ; \mathbf{c})$.

Before going further, let us consider the properties of such curves and the motivation for the definition. Observe first that the combination of ind $(u ; \mathbf{c}) \geq 0$ and the relation

$$
2 c_{N}(u ; \mathbf{c})=\operatorname{ind}(u ; \mathbf{c})-2+2 g+\# \Gamma_{0}(\mathbf{c})
$$

gives the lower bound $c_{N}(u ; \mathbf{c}) \geq-1$. Then the adjunction formula together with $i(u ; \mathbf{c} \mid u ; \mathbf{c}) \leq 0$ implies $\operatorname{sing}(u ; \mathbf{c})=0$, so every somewhere injective curve in $\mathcal{M}_{u}^{\mathbf{c}}$ is embedded. We can also deduce from the adjunction formula that $c_{N}(u ; \mathbf{c}) \leq 0$, and then (4.11) implies ind $(u ; \mathbf{c}) \leq 2$. The index 1 and 2 cases are of particular interest: since $\# \Gamma_{0}(\mathbf{c})$ and ind $(u ; \mathbf{c})$ always have the same parity due to the index formula, it follows from (4.11) that curves of index 1 or 2 satisfying our conditions have $c_{N}(u ; \mathbf{c})=0$ and thus $i(u ; \mathbf{c} \mid u ; \mathbf{c})=0$. The transversality criterion $\operatorname{ind}(u ; \mathbf{c})>c_{N}(u ; \mathbf{c})$ is clearly satisfied, and thus $u$ lives in a 1 or 2-dimensional family of embedded curves that never intersect each other. These are precisely the curves that appear in $J$-holomorphic foliations of $W$, or in the case where $W$ is a symplectization $\mathbb{R} \times M$, the finite energy foliations of Hofer, Wysocki and Zehnder [HWZ03]. Isolated curves with ind $(u ; \mathbf{c})=0$ can also occur in such foliations (surrounded by families of larger index): we shall show for instance that stable, nicely embedded index 0 curves appear as the underlying somewhere injective curves when families of larger index degenerate to multiple covers.

It will be useful to note that due to (4.11), all stable nicely embedded curves also have the following properties:

(1) $g=0$,

(2) $\# \Gamma_{0}(\mathbf{c})=1$ if $\operatorname{ind}(u ; \mathbf{c})=1$, and otherwise $\# \Gamma_{0}(\mathbf{c})=0$. 
In the cases $\operatorname{ind}(u ; \mathbf{c})=1$ or 2 , we have observed that $c_{N}(u ; \mathbf{c})=0$ and thus the adjunction formula also implies $\operatorname{cov}_{\infty}\left(\gamma_{\Gamma} ; \mathbf{c}\right)=\operatorname{cov}_{\mathrm{MB}}\left(\gamma_{\Gamma} ; \mathbf{c}\right)=0$. We will use this shortly to prove the following consequence for the unique even puncture $z \in \Gamma_{0}(\mathbf{c})$ in the index 1 case:

Proposition 4.13. If $u \in \mathcal{M}^{\mathbf{c}}$ is a stable, nicely embedded curve with ind $(u ; \mathbf{c})=1$, then the unique even puncture $z \in \Gamma_{0}^{ \pm}$(c) satisfies one of the following:

(1) $\gamma_{z}$ is nondegenerate and even,

(2) $\gamma_{z}$ belongs to a 2-dimensional Morse-Bott manifold, and $\nu_{\mp}\left(\gamma_{z}\right)=0$ if and only if $z \in \Gamma_{C}$.

Moreover, $\gamma_{z}$ is either simply covered, or is doubly covered such that the underlying simple orbit is nondegenerate and odd.

Definition 4.14. Adapting some terminology from Symplectic Field Theory [EGH00], we will call $z \in \Gamma^{ \pm}$a bad puncture if $z \in \Gamma_{0}(\mathbf{c})$ and $\gamma_{z}=\gamma^{2}$ for some nondegenerate odd orbit $\gamma$.

Remark 4.15. In this terminology, Proposition 4.13 says that the unique even puncture has an orbit of covering number 1 or 2 , and is bad in the latter case. In SFT of course, "bad" also means "to be ignored": moduli spaces of curves with such punctures cannot be oriented, but they also need not be counted in constructing the algebra of the theory.

This is enough preparation to state the strong version of Theorem 2. In the following, we use expressions such as "for generic $J \ldots$.." or " $J$ is generic" to mean more precisely: "there exists a Baire subset $\mathcal{g} \subset \mathcal{H}_{\omega}\left(W, \mathscr{H}_{+}, \mathscr{H}_{-}\right)$such that the following is true if $J \in \mathcal{L}$." Similarly, "for generic homotopies..." means that there exists a Baire subset in the space of smooth homotopies in $\mathscr{g}_{\omega}\left(W, \mathscr{H}_{+}, \mathscr{H}_{-}\right)$for which the statement is true.

Theorem 4. Assume $\left\{J_{\tau}\right\}_{\tau \in[0,1]}$ is a smooth 1-parameter family of almost complex structures in $\mathcal{L}_{\omega}\left(W, \mathscr{H}_{+}, \mathscr{H}_{-}\right)$such that either

(1) the homotopy $\tau \mapsto J_{\tau}$ is generic, or

(2) $J_{\tau}=J$ is independent of $\tau$ and is generic.

Suppose $\tau_{n} \rightarrow \tau_{\infty} \in[0,1]$ and $u_{n}: \dot{\Sigma} \rightarrow W$ is a sequence of asymptotically cylindrical $J_{\tau_{n}}$-holomorphic curves, which are stable and nicely embedded with respect to some fixed asymptotic constraints $\mathbf{c}$ and converge to a smooth $J_{\tau_{\infty}}$-holomorphic curve $u: \dot{\Sigma} \rightarrow W$. Then:

- If $\operatorname{ind}(u ; \mathbf{c})=0$ or ind $(u ; \mathbf{c})=1$ with $\gamma_{z}$ simply covered for the unique even puncture $z \in \Gamma_{0}(\mathbf{c})$, then $u$ is a stable, nicely embedded curve. 
- If $\operatorname{ind}(u ; \mathbf{c})=1$ and the unique even puncture $z \in \Gamma_{0}(\mathbf{c})$ is bad (with $\gamma_{z}$ doubly covered $)$, or $\operatorname{ind}(u ; \mathbf{c})=2$, then $u$ is either a stable, nicely embedded curve or an unbranched multiple cover of a stable, nicely embedded index 0 curve.

In all cases, $u$ is regular.

Note that since $\operatorname{sing}(u ; \mathbf{c})=0$ for all stable, nicely embedded curves, the MorseBott contribution $\delta_{\mathrm{MB}}^{ \pm}\left(\gamma_{z}\right)$ also vanishes at each puncture. Plugging in (4.8) leads immediately to the following consequence:

Lemma 4.16. For any stable, nicely embedded curve $u$, if $z \in \Gamma_{U}^{ \pm}$is an unconstrained puncture with a degenerate orbit $\gamma_{z}$ which is exceptional in the sense of Proposition 4.1, then $\nu_{\mp}\left(\gamma_{z}\right)=0$ and $\operatorname{cov}_{\mp}\left(\gamma_{z}\right)=\operatorname{cov}_{\mp}\left(\gamma_{z}^{\epsilon}\right)$.

Proof of Proposition 4.13. The first alternative follows easily from the formula $p\left(\gamma_{z} \pm \mathbf{c}_{z}\right)=\alpha_{+}^{\Phi}\left(\gamma_{z} \pm \mathbf{c}_{z}\right)-\alpha_{-}^{\Phi}\left(\gamma_{z} \pm \mathbf{c}_{z}\right)$ and the definition of $\nu_{\mp}\left(\gamma_{z}\right)$. We have also $\operatorname{cov}_{\mp}\left(\gamma_{z}^{\epsilon}\right)=1$ and $\left[\operatorname{cov}\left(\gamma_{z}^{\epsilon}\right)-1\right] \cdot \nu_{\mp}\left(\gamma_{z}\right)=0$, implying the same statements for $\gamma_{z}$ due to Lemma 4.16.

If $\gamma_{z}$ is nondegenerate, we claim now that it cannot be a multiple cover of any even orbit $\gamma^{\prime}$. Otherwise there are eigenfunctions $e_{ \pm}$of $\boldsymbol{A}_{\gamma^{\prime}}$ with identical winding numbers and eigenvalues of opposite sign, so the corresponding covers give a pair of neighboring eigenfunctions in the spectrum of $\boldsymbol{A}_{\gamma_{z}}$; their eigenvalues are therefore the largest negative and smallest positive elements of $\sigma\left(\boldsymbol{A}_{\gamma_{z}}\right)$, implying $\operatorname{cov}_{\mp}\left(\gamma_{z}\right)>1$, a contradiction. This leaves two possibilities for the simply covered orbit underlying $\gamma_{z}$ : it is either even (and thus is $\gamma_{z}$ itself) or is odd but hyperbolic, in which case $\gamma_{z}$ can only be its double cover.

In the Morse-Bott case, suppose first that $\nu_{\mp}\left(\gamma_{z}\right)=0$. Then any section whose covering number is counted by $\operatorname{cov}_{\mp}\left(\gamma_{z}^{\epsilon}\right)$ has the same winding and hence the same covering number as a section in $\operatorname{ker} \boldsymbol{A}_{\gamma_{z}^{\epsilon}}$, thus also the same covering number as $\gamma_{z}^{\epsilon}$ itself. We conclude that $\gamma_{z}^{\epsilon}$ is simply covered, so either $\gamma_{z}$ is as well or it is an exceptional orbit with isotropy 2 as described in Proposition 4.1. The same result follows if $v_{\mp}\left(\gamma_{z}\right)=1$ because $\left[\operatorname{cov}\left(\gamma_{z}\right)-1\right] \cdot v_{\mp}\left(\gamma_{z}\right)$.

In the proof of Theorem 4, we shall need the following small variation on the usual implicit function theorem:

Lemma 4.17. Suppose $f: X \rightarrow Y$ is a smooth Fredholm map between Banach spaces with $f(0)=0$, and $Q \subset X$ is a smooth finite dimensional submanifold of $X$ that contains 0 , is contained in $f^{-1}(0)$ and satisfies

$$
\operatorname{dim} \operatorname{ker} d f(0)=\operatorname{dim} Q .
$$

Then $Q$ also contains a neighborhood of 0 in $f^{-1}(0)$; in particular this neighborhood is a smooth manifold of dimension $\operatorname{dim} \operatorname{ker} d f(0)$. 
Proof. Let $V=\operatorname{im} d f(0) \subset Y$ and choose a linear projection map $\pi_{V}: Y \rightarrow V$ along some closed complement. Then $\pi_{V} \circ f: X \rightarrow V$ is also Fredholm and is regular at 0 , so the implicit function theorem gives $\left(\pi_{V} \circ f\right)^{-1}(0)$ near 0 the structure of a smooth manifold of dimension $\operatorname{dim} \operatorname{ker} d f(0)$. Now

$$
Q \subset f^{-1}(0) \subset\left(\pi_{V} \circ f\right)^{-1}(0),
$$

where the spaces on the left and right are manifolds of the same dimension containing 0 ; the result follows.

To every connected component of the moduli space $\mathcal{M}^{\mathbf{c}}$, one can associate the data $\left(\Sigma, \Gamma, P_{\Gamma}\right)$, where $\Sigma \backslash \Gamma$ is the domain of any curve in the component (well defined up to diffeomorphism) and $P_{\Gamma}$ is the collection of orbits and/or Morse-Bott submanifolds $\left\{P_{z}\right\}_{z \in \Gamma}$ that determine the asymptotic behavior of such a curve. Let us introduce the notation

$$
\mathcal{M}\left(\Sigma, \Gamma, P_{\Gamma}\right) \subset \mathcal{M}^{\mathbf{c}}
$$

to indicate the union of all connected components of $\mathcal{M}^{\mathbf{c}}$ that have this particular domain and asymptotic behavior.

Lemma 4.18. For any component $\mathcal{M}\left(\Sigma, \Gamma, P_{\Gamma}\right) \subset \mathcal{M}^{\mathbf{c}}$, there exists a finite set $\mathcal{C}$ containing tuples $\left(\Sigma^{\prime}, \Gamma^{\prime}, P_{\Gamma^{\prime}}, \mathbf{c}^{\prime}\right)$ such that the following is true: if $u=v \circ \varphi \in$ $\mathcal{M}\left(\Sigma, \Gamma, P_{\Gamma}\right)$ is a multiple cover and $v$ is the underlying somewhere injective curve, then there exists $\left(\Sigma^{\prime}, \Gamma^{\prime}, P_{\Gamma^{\prime}}, \mathbf{c}^{\prime}\right) \in \mathcal{C}$ such that $v \in \mathcal{M}\left(\Sigma^{\prime}, \Gamma^{\prime}, P_{\Gamma^{\prime}}\right) \subset \mathcal{M}^{\mathbf{c}^{\prime}}$ and $\mathbf{c} \leq \varphi^{*} \mathbf{c}^{\prime}$ in the sense of Definition 4.10.

Proof. The Riemann-Hurwitz formula constrains the genus of $\Sigma^{\prime}$ to be less than or equal to that of $\Sigma$, allowing only finitely many different closed surfaces. Having chosen $\Sigma^{\prime}$, the relation $\gamma_{z}=\gamma_{\varphi(z)}^{k_{z}}$ for $z \in \Gamma$ and $k_{z}:=\operatorname{ord}(d \varphi ; z)+1$ allows $k_{z}$ to vary between 1 and $\operatorname{cov}\left(\gamma_{z}\right)$, thus giving a finite range of choices for each puncture. After making this choice, we can also decide which punctures $z, z^{\prime} \in \Gamma$ might have the same image under $\varphi$ : this is allowed only when $P_{z}$ and $P_{z^{\prime}}$ belong to the same Morse-Bott manifold, and again presents a finite range of choices. The number of punctures $\Gamma^{\prime}$ and their asymptotic limits $P_{\Gamma^{\prime}}$ are uniquely determined by this choice. Finally, the constraints $\mathbf{c}^{\prime}$ can be defined as follows: for any constrained $z \in \Gamma$, define $\zeta:=\varphi(z)$ to be a constrained puncture, fixed at the unique orbit $\gamma_{\zeta}$ such that $\gamma_{z}=\gamma_{\zeta}^{k_{z}}$. Any puncture $\zeta \in \Gamma^{\prime}$ not touched by this algorithm will be considered unconstrained. By construction now, $\mathbf{c} \leq \varphi^{*} \mathbf{c}^{\prime}$.

Proof of Theorem 4. We will carry out the proof in several steps assuming $\left\{J_{\tau}\right\}_{\tau \in[0,1]}$ is a generic homotopy; the proof for a fixed generic $J$ is the same but slightly simpler in a few details. 
If $u$ is somewhere injective there's nothing to prove, so assume $u=v \circ \varphi$ for a somewhere injective curve $v: \dot{\Sigma}^{\prime} \rightarrow W$ and a holomorphic branched cover $\varphi: \Sigma \rightarrow$ $\Sigma^{\prime}$ of degree $k \geq 2$. By Lemma 4.18, the domain of $v$ is one out of a finite set of choices and satisfies constraints $\mathbf{c}^{\prime}$ with $\mathbf{c} \leq \varphi^{*} \mathbf{c}^{\prime}$. For each such choice, there exists a generic set of homotopies $\left\{J_{\tau}\right\}$ such that we can assume ind $\left(v ; \mathbf{c}^{\prime}\right) \geq-1$, and the intersection of all these generic sets is also generic, hence the genericity assumption implies ind $\left(v ; \mathbf{c}^{\prime}\right) \geq-1$. By (4.11) then, $c_{N}\left(v ; \mathbf{c}^{\prime}\right) \geq-1$.

Step 1. We show that $c_{N}\left(v ; \mathbf{c}^{\prime}\right)=-1$. Combining Proposition 4.8 and Proposition 4.11 yields

$$
0 \geq c_{N}(u ; \mathbf{c}) \geq c_{N}\left(u ; \varphi^{*} \mathbf{c}^{\prime}\right)=k c_{N}\left(v ; \mathbf{c}^{\prime}\right)+Z(d \dot{\varphi})+Q,
$$

so the only other alternative is $c_{N}\left(v ; \mathbf{c}^{\prime}\right)=0$, in which case $c_{N}(u ; \mathbf{c})=Z(d \dot{\varphi})=$ $Q=0$. Then all critical points of $\varphi: \Sigma \rightarrow \Sigma^{\prime}$ are at punctures, and the RiemannHurwitz formula gives $2 k-2$ of them (counting multiplicity) since both $\Sigma$ and $\Sigma^{\prime}$ necessarily have genus zero. Denote $k_{z}=\operatorname{ord}(d \varphi ; z)+1 \in \mathbb{N}$ for each $z \in \Gamma$. Now $Q=0$ implies that for each $z \in \Gamma^{ \pm}$, if $\zeta=\varphi(z)$,

$$
\alpha_{\mp}^{\Phi}\left(\gamma_{z} \pm\left(\varphi^{*} \mathbf{c}^{\prime}\right)_{z}\right)=\alpha_{\mp}^{\Phi}\left(\gamma_{\zeta}^{k_{z}} \pm\left(\varphi^{*} \mathbf{c}^{\prime}\right)_{z}\right)=k_{z} \alpha_{\mp}^{\Phi}\left(\gamma_{\zeta} \pm \mathbf{c}_{\zeta}^{\prime}\right),
$$

so depending on the constraints, we have either $\alpha_{\mp}^{\Phi}\left(\gamma_{z}\right) \in k_{z} \mathbb{Z}$ or $\alpha_{\mp}^{\Phi}\left(\gamma_{z} \mp \boldsymbol{\delta}\right) \in k_{z} \mathbb{Z}$, the latter only if $\zeta \in \Gamma_{U}^{\prime}$, which implies $z \in \Gamma_{U}$. In either case, Lemma 4.2 then implies that a certain eigenfunction $e$ of $\boldsymbol{A}_{\gamma_{z}}$ is a $k_{z}$-fold cover. If it is the first case, then $\operatorname{cov}_{\mp}\left(\gamma_{z}\right) \geq k_{z}$, and this equals $\operatorname{cov}_{\mp}\left(\gamma_{z}^{\epsilon}\right)$ by Lemma 4.16. In the second case, we have $\operatorname{cov}\left(\gamma_{z}\right) \geq k_{z}$ and $e \in \operatorname{ker} \boldsymbol{A}_{\gamma_{z}}$. If $\nu_{\mp}\left(\gamma_{z}\right)=0$, then $\operatorname{wind}^{\Phi}(e)=$ $\alpha_{\mp}^{\Phi}\left(\gamma_{z}\right) \in k_{z} \mathbb{Z}$, so Lemmas 4.2 and 4.16 again imply $\operatorname{cov}_{\mp}\left(\gamma_{z}^{\epsilon}\right)=\operatorname{cov}_{\mp}\left(\gamma_{z}\right) \geq$ $k_{z}$. Otherwise $\nu_{\mp}\left(\gamma_{z}\right)=1$, so Lemma 4.16 implies that $\gamma_{z}$ is generic and thus $v_{\mp}\left(\gamma_{z}\right) \cdot\left[\operatorname{cov}\left(\gamma_{z}^{\epsilon}\right)-1\right]=v_{\mp}\left(\gamma_{z}\right) \cdot\left[\operatorname{cov}\left(\gamma_{z}\right)-1\right] \geq k_{z}-1$. Putting all of these cases together and summing over $z \in \Gamma$, we find

$$
\operatorname{cov}_{\infty}\left(\gamma_{\Gamma} ; \mathbf{c}\right)+\operatorname{cov}_{\mathrm{MB}}\left(\gamma_{\Gamma} ; \mathbf{c}\right) \geq \sum_{z \in \Gamma}\left(k_{z}-1\right)=2 k-2 \geq 2 .
$$

Thus for large $n, i\left(u_{n} ; \mathbf{c} \mid u_{n} ; \mathbf{c}\right)=2 \operatorname{sing}\left(u_{n} ; \mathbf{c}\right)+c_{N}\left(u_{n} ; \mathbf{c}\right)+\operatorname{cov}_{\infty}\left(\gamma_{\Gamma} ; \mathbf{c}\right)+$ $\operatorname{cov}_{\mathrm{MB}}\left(\gamma_{\Gamma} ; \mathbf{c}\right) \geq 2$, a contradiction.

In light of this result and (4.11), we have either ind $\left(v ; \mathbf{c}^{\prime}\right)=0$ with all punctures odd or ind $\left(v ; \mathbf{c}^{\prime}\right)=-1$ with exactly one even puncture.

Step 2. Claim ind $\left(v ; \mathbf{c}^{\prime}\right)=0$. If not, then $\operatorname{ind}\left(v ; \mathbf{c}^{\prime}\right)=-1$ and $\# \Gamma_{0}^{\prime}\left(\mathbf{c}^{\prime}\right)=1$, and since covers of even orbits are always even (Lemma 4.3), \# $\Gamma_{0}(\mathbf{c}) \geq 1$, implying $\operatorname{ind}(u ; \mathbf{c})=1$. In this case $u$ also has exactly one even puncture $z \in \Gamma_{0}(\mathbf{c})$, so $\gamma_{z}=\gamma_{\zeta}^{k}$ with $\zeta:=\varphi(z) \in \Gamma_{0}^{\prime}\left(\mathbf{c}^{\prime}\right)$. There are now three cases to consider:

(1) If $\gamma_{\zeta}$ is nondegenerate, then so is $\gamma_{z}$ and its extremal eigenfunctions are the $k$-fold covers of those of $\gamma_{\zeta}$, giving $\operatorname{cov}_{\mp}\left(\gamma_{z}^{\epsilon}\right) \geq k$. 
(2) If $\gamma_{\zeta}$ is Morse-Bott with $\nu_{\mp}\left(\gamma_{\zeta}\right)=0$, then the extremal eigenfunction of a generic perturbation $\gamma_{\zeta}^{\epsilon}$ has the same winding and thus same covering number as a section in $\operatorname{ker} \boldsymbol{A}_{\gamma_{\zeta}^{\epsilon}}$, and the same is true for the $k$-fold cover. Moreover, the Morse-Bott family containing $\gamma_{z}^{\epsilon}$ is at least $k$-fold covered, which implies the same for sections in $\operatorname{ker} \boldsymbol{A}_{\gamma_{z}^{\epsilon}}$. So again, $\operatorname{cov}_{\mp}\left(\gamma_{z}^{\epsilon}\right)=\operatorname{cov}\left(\gamma_{z}^{\epsilon}\right) \geq k$.

(3) If $\nu_{\mp}\left(\gamma_{\zeta}\right)=1$, then Lemma 4.16 implies $\gamma_{z}$ is generic, so we can take $\gamma_{z}^{\epsilon}=\gamma_{z}$ without loss of generality and conclude $\left[\operatorname{cov}\left(\gamma_{z}^{\epsilon}\right)-1\right] \cdot \nu_{\mp}\left(\gamma_{z}\right) \geq k$.

The conclusion from all of these cases is that $\operatorname{cov}_{\infty}\left(\gamma_{\Gamma} ; \mathbf{c}\right)+\operatorname{cov}_{\mathrm{MB}}\left(\gamma_{\Gamma} ; \mathbf{c}\right) \geq k-1 \geq$ 1 , and since $c_{N}(u ; \mathbf{c})=0$ for the index 1 case, a contradiction arises again from the adjunction formula: $i\left(u_{n} ; \mathbf{c} \mid u_{n} ; \mathbf{c}\right)=2 \operatorname{sing}\left(u_{n} ; \mathbf{c}\right)+c_{N}\left(u_{n} ; \mathbf{c}\right)+\operatorname{cov}_{\infty}\left(\gamma_{\Gamma} ; \mathbf{c}\right)+$ $\operatorname{cov}_{\mathrm{MB}}\left(\gamma_{\Gamma} ; \mathbf{c}\right) \geq 1$.

Step 3. Since $0=\operatorname{ind}\left(v ; \mathbf{c}^{\prime}\right)>c_{N}\left(v ; \mathbf{c}^{\prime}\right)=-1$, it now follows immediately from Proposition 4.9 and Proposition 4.11 that $v$ is a stable, nicely embedded curve, as

$$
0 \geq i(u ; \mathbf{c} \mid u ; \mathbf{c}) \geq i\left(u ; \varphi^{*} \mathbf{c}^{\prime} \mid u ; \varphi^{*} \mathbf{c}^{\prime}\right) \geq k^{2} \cdot i\left(v ; \mathbf{c}^{\prime} \mid v ; \mathbf{c}^{\prime}\right) .
$$

Step 4. If ind $(u ; \mathbf{c})=1$, then its unique even orbit cannot be simply covered since $v$ has only odd orbits. Thus the even orbit must be a doubly covered orbit at a bad puncture.

Step 5. We claim $u$ is immersed and has ind $(u ; \mathbf{c})>0$. Suppose not, i.e. that either $Z(d u)>0$ or $\operatorname{ind}(u ; \mathbf{c})=0$. Then $\operatorname{ind}(u ; \mathbf{c}) \leq 2 Z(d u)$, so Theorem 1 gives

$$
\begin{aligned}
2 Z(d u) & \leq \operatorname{dim} \operatorname{ker}\left(D \bar{\partial}_{\tau_{\infty}}(j, u) / \operatorname{aut}(\dot{\Sigma}, j)\right) \\
& \leq 2 Z(d u)+K\left(c_{N}(u ; \mathbf{c})-Z(d u), \# \Gamma_{0}(\mathbf{c})\right)=2 Z(d u)
\end{aligned}
$$

since $c_{N}(u ; \mathbf{c})-Z(d u)<0$. Extending the usual bundle on $\mathcal{T} \times \mathscr{B}$ to allow parametrized $J$, we can now consider a nonlinear operator

$$
\bar{\partial}:[0,1] \times \mathcal{T} \times \mathcal{B} \rightarrow \mathcal{E}, \quad\left(\tau, j^{\prime}, u^{\prime}\right) \mapsto \bar{\partial}_{J_{\tau}}\left(j^{\prime}, u^{\prime}\right) .
$$

Since $v$ is embedded, every critical point of $u$ arises as a branch point of $\dot{\varphi}: \dot{\Sigma} \rightarrow \dot{\Sigma}^{\prime}$, thus $Z(d u)=Z(d \dot{\varphi})$, and (4.12) now implies

$$
\operatorname{dim} \operatorname{ker} D \bar{\partial}\left(\tau_{\infty}, j, u\right) \leq 2 Z(d \dot{\varphi})+\operatorname{dim} \operatorname{Aut}(\dot{\Sigma}, j)+1 .
$$

To apply Lemma 4.17, we shall now find a smooth manifold of precisely this dimension that is contained in $\bar{\partial}^{-1}(0)$. The key is to look at the space of holomorphic branched covers $\dot{\Sigma} \rightarrow \dot{\Sigma}^{\prime}$ close to $\dot{\varphi}$. Observe that since $v$ is embedded and satisfies the transversality criterion $0=\operatorname{ind}\left(v ; \mathbf{c}^{\prime}\right)>c_{N}\left(v ; \mathbf{c}^{\prime}\right)=-1$, for $\tau$ close to $\tau_{\infty}$ we obtain from the implicit function theorem a smooth 1-parameter family of asymptotically cylindrical pseudoholomorphic maps

$$
v_{\tau}:\left(\dot{\Sigma}^{\prime}, j_{\tau}\right) \rightarrow\left(W, J_{\tau}\right)
$$


satisfying the constraints $\mathbf{c}^{\prime}$, with $v_{\tau_{\infty}}=v$. The holomorphic maps from $\dot{\Sigma}$ to $\left(\dot{\Sigma}^{\prime}, j_{\tau}\right)$ can then be identified with the zero-set of a section

$$
\bar{\partial}_{\Sigma^{\prime}}:[0,1] \times \mathcal{T} \times \mathcal{B}^{\Sigma^{\prime}} \rightarrow \mathcal{E}^{\Sigma^{\prime}}, \quad\left(\tau, j^{\prime}, \varphi^{\prime}\right) \mapsto T \varphi^{\prime}+j_{\tau} \circ T \varphi^{\prime} \circ j^{\prime},
$$

and by the remarks in Example 3.16, a neighborhood of $\dot{\varphi}$ in $\bar{\partial} \bar{\Sigma}^{\prime}(0)$ is a smooth manifold of dimension $2 Z(\dot{\varphi})+\operatorname{Aut}(\dot{\Sigma}, j)+1$. Now for $\left(\tau, j^{\prime}, \varphi^{\prime}\right) \in \bar{\partial}_{\Sigma^{\prime}}^{-1}(0)$, we have $\left(\tau, j^{\prime}, v_{\tau} \circ \varphi^{\prime}\right) \in \bar{\partial}^{-1}(0)$, thus embedding $\bar{\partial}_{\Sigma^{\prime}}^{-1}(0)$ as a smooth submanifold of dimension $2 Z(d \dot{\varphi})+\operatorname{dim} \operatorname{Aut}(\dot{\Sigma}, j)+1$ in $\bar{\partial}^{-1}(0)$. It follows that (4.13) is an equality, and Lemma 4.17 now implies that every element of $\bar{\partial}^{-1}(0)$ near $\left(\tau_{\infty}, j, u\right)$ belongs to this submanifold; this is a contradiction, as it implies that for large $n, u_{n}$ must also be a multiple cover.

Step 6. Since we now know that $u$ is immersed, it is immediate from Theorem 1 that $u$ is regular.

Corollary 4.19. For generic $J$, if $u \in \mathcal{M}^{\mathbf{c}}$ is a stable, nicely embedded $J$-holomorphic curve then $\mathcal{M}_{u}^{\mathbf{c}}$ is a smooth orbifold of dimension ind $(u ; \mathbf{c})$ with only isolated singularities. Moreover, the images of any two curves in $\mathcal{M}_{u}^{\mathbf{c}}$ are either identical or disjoint, and they are all stable and nicely embedded except for a discrete set of unbranched multiple covers of embedded curves.

Proof. The statement about the images follows from positivity of intersections and the condition $i(u ; \mathbf{c} \mid u ; \mathbf{c}) \leq 0$. The only remaining part not immediate from Theorem 4 is that the multiple covers are isolated; this is related to the fact that the orbifold singularities must be isolated for orientation reasons (see Remark 4.21 below), but does not follow from it. So, we claim that for any multiple cover $u=v \circ \varphi$ arising as a limit in the theorem, every other curve close to $u$ is somewhere injective. Here we can assume ind $(u ; \mathbf{c})$ is 1 or 2 , so $c_{N}(u ; \mathbf{c})=\operatorname{cov}_{\infty}\left(\gamma_{\Gamma} ; \mathbf{c}\right)=\operatorname{cov}_{\mathrm{MB}}\left(\gamma_{\Gamma} ; \mathbf{c}\right)=0$. Now note that Theorem 3 and the implicit function theorem give a natural isomorphism $T_{u} \mathcal{M}^{\mathbf{c}}=\operatorname{ker} \boldsymbol{D}_{u}^{N}$ since $u$ is immersed, and it will thus suffice to show that nontrivial sections $\eta \in \operatorname{ker} \boldsymbol{D}_{u}^{N} \subset \Gamma\left(N_{u}\right)$ are not multiply covered. Otherwise, using the natural identification $N_{u}=\varphi^{*} N_{v}$, there exists a nonzero $\eta \in \operatorname{ker} \boldsymbol{D}_{u}^{N}$ and a section $\eta^{\prime} \in \Gamma\left(N_{v}\right)$ such that $\eta=\eta^{\prime} \circ \varphi$. We know that $\eta$ is zero free (also at infinity), since using (2.7), Proposition 3.18 and the usual identification of $\boldsymbol{D}_{u}^{N}$ with an operator on non-weighted spaces,

$$
Z(\eta)+Z_{\infty}(\eta)=c_{1}\left(N_{u}, \ell_{N}, A_{\Gamma} \pm \mathbf{c}_{\Gamma}\right)=c_{N}(u ; \mathbf{c})=0
$$

This implies that the winding of $\eta$ near each puncture $z \in \Gamma^{ \pm}$attains the extremal value $\alpha_{\mp}^{\Phi}\left(\gamma_{z} \pm \mathbf{c}_{z}\right)$. But this is impossible if $\eta=\eta^{\prime} \circ \varphi$ : indeed, the fact that $\dot{\varphi}: \dot{\Sigma} \rightarrow \dot{\Sigma}^{\prime}$ is immersed but both surfaces have genus zero implies that there exists 
a puncture $z \in \Gamma$ at which $\varphi$ has nontrivial branching order $k_{z}:=\operatorname{ord}(d \varphi ; z)>1$, so the asymptotic winding wind ${ }_{z}^{\Phi}(\eta)$ of $\eta$ near $z$ satisfies

$$
\begin{aligned}
\pm \operatorname{wind}_{z}^{\Phi}(\eta) & \leq \pm k_{z} \alpha_{\mp}^{\Phi}\left(\gamma_{\varphi(z)} \pm \mathbf{c}_{\varphi(z)}^{\prime}\right) \\
& = \pm \alpha_{\mp}^{\Phi}\left(\gamma_{z} \pm\left(\varphi^{*} \mathbf{c}^{\prime}\right)_{\varphi(z)}\right)-q_{\mp}\left(\gamma_{\varphi(z)} \pm \mathbf{c}_{\varphi(z)}^{\prime} ; k_{z}\right) \\
& \leq \pm \alpha_{\mp}^{\Phi}\left(\gamma_{z} \pm \mathbf{c}_{z}\right)-q_{\mp}\left(\gamma_{\varphi(z)} \pm \mathbf{c}_{\varphi(z)}^{\prime} ; k_{z}\right),
\end{aligned}
$$

implying $q_{\mp}\left(\gamma_{\varphi(z)} \pm \mathbf{c}_{\varphi(z)}^{\prime} ; k_{z}\right)=0$ and thus $\alpha_{\mp}^{\Phi}\left(\gamma_{z} \pm \mathbf{c}_{z}\right) \in k_{z} \mathbb{Z}$. Then repeating an argument that is by now familiar from the proof of Theorem 4 , we find a contradiction in the form $\operatorname{cov}_{\infty}\left(\gamma_{\Gamma} ; \mathbf{c}\right)+\operatorname{cov}_{\mathrm{MB}}\left(\gamma_{\Gamma} ; \mathbf{c}\right)>0$.

Remark 4.20. It is shown in [Wenb] that if $u: \dot{\Sigma} \rightarrow W$ is a stable nicely embedded index 2 curve, then the nearby curves in $\mathcal{M}_{u}^{\mathbf{c}}$ foliate a neighborhood of $u(\dot{\Sigma})$ in $W$. Now suppose $u$ is a multiply covered index 2 curve that is a limit of stable nicely embedded curves. Then since $u$ is immersed and $T_{u} \mathcal{M}^{\mathbf{c}}$ consists of zero-free sections of its normal bundle, the same argument shows that the nearby curves in $\mathcal{M}_{u}^{\mathbf{c}}$ again foliate a neighborhood of $u(\dot{\Sigma})$. In this foliation, $u(\dot{\Sigma})$ is an exceptional leaf, being the embedded image of an isolated index 0 curve. An explicit example is constructed below.

Remark 4.21. The fact that singularities in a 1-dimensional orbifold are isolated is obvious, and in two dimensions it is true if the orbifold is oriented, as an oriented orbifold can only have singularities of codimension at least two. By results in [BM04], $\mathcal{M}_{u}$ does admit an orientation if $u$ is a stable, nicely embedded curve of index 2 , and the same is true for index 1 if and only if the unique even puncture is not a "bad" puncture. This excludes singularities in the index 1 case entirely unless the even puncture is bad, and indeed, we have shown that multiple covers do not appear in this case. These remarks are not quite enough to prove Corollary 4.19 however, as in general there can be multiple covers with trivial automorphism groups, which therefore do not cause singularities.

Example 4.22. We now consider a concrete situation in which nicely embedded curves of index 2 are seen to converge to an isolated, unbranched multiple cover.

Identify $S^{2}$ with the extended complex plane and let

$$
W=\left(S^{2} \times S^{2}\right) \backslash\{(0,0),(\infty, \infty),(1,1)\},
$$

choosing the standard complex structure $J=i \oplus i$. This can be regarded as a manifold with three negative cylindrical ends asymptotic to the standard contact 3sphere, whose Reeb orbits are the fibers of the Hopf fibration. The asymptotics are therefore Morse-Bott: there is a 2-dimensional family of closed orbits at each end. 
Now for $\zeta \in \mathbb{C} \backslash\{0,-1,1\}$, consider the 2-dimensional family of $J$-holomorphic four-punctured spheres

$$
u_{\zeta}: S^{2} \backslash\{0,1,-1, \infty\} \rightarrow W, \quad z \mapsto\left(z^{3} \frac{z+\zeta}{\zeta z+1}, z^{2}\right) .
$$

These are all proper and embedded, with asymptotic behavior as follows:

- At $0, u_{\zeta}$ is asymptotic to a fixed doubly covered orbit in the end $(0,0)$.

- At $\infty, u_{\zeta}$ is asymptotic to a fixed doubly covered orbit in the end $(\infty, \infty)$.

- At 1 and $-1, u_{\zeta}$ is asymptotic to an arbitrary (not fixed) simply covered orbit in the end $(1,1)$.

One can use the setup we have described to show that the moduli space of embedded holomorphic curves satisfying precisely these asymptotic constraints and representing the same relative homology class is indeed a smooth 2-dimensional manifold: indeed, $\operatorname{ind}(u ; \mathbf{c})=2$ and $i(u ; \mathbf{c} \mid u ; \mathbf{c})=c_{N}(u ; \mathbf{c})=\operatorname{cov}_{\infty}\left(\gamma_{\Gamma} ; \mathbf{c}\right)=\operatorname{cov}_{\mathrm{MB}}\left(\gamma_{\Gamma} ; \mathbf{c}\right)=0$. Now as $\zeta \rightarrow 0$, the family converges to the curve $u_{0}(z)=\left(z^{4}, z^{2}\right)=: v\left(z^{2}\right)$, an unbranched double cover of the embedded 3-punctured sphere

$$
v: S^{2} \backslash\{0,1, \infty\} \rightarrow W, \quad z \mapsto\left(z^{2}, z\right),
$$

and with the appropriate asymptotic constraints $\mathbf{c}^{\prime}$ one can indeed show that $\operatorname{ind}\left(v ; \mathbf{c}^{\prime}\right)=0$ and $i\left(v ; \mathbf{c}^{\prime} \mid v ; \mathbf{c}^{\prime}\right)=c_{N}\left(v ; \mathbf{c}^{\prime}\right)=-1$. Observe that the images of $u_{\zeta}$ for $\zeta$ near zero together with the image of $v$ foliate a neighborhood of $v$ in $W$. Due to the ordering of the punctures, $\operatorname{Aut}\left(u_{0}\right)$ is the trivial group, so the moduli space remains a smooth manifold even with $u_{0}$ included. If we take the quotient of this space by forgetting the order of the punctures, it becomes a smooth orbifold in which $u_{0}$ has isotropy group $\mathbb{Z}_{2}$.

Remark 4.23. It is also interesting to see what happens to the family $u_{\zeta}$ as $\zeta \rightarrow \pm 1$ or $\zeta \rightarrow \infty$ : here it turns out that $u_{\zeta}$ breaks into a $J$-holomorphic building (in the sense of the SFT compactness theorem [BEH+03]). At $\zeta \rightarrow \infty$ in particular, the building includes a component that is an unbranched multiple cover of index -2 over a nicely embedded index 0 curve. It is work in progress by the author to generalize Theorem 4 in light of SFT compactness and show that such behavior is quite general: indeed, that only unbranched multiple covers can arise in such limits, and that there exists a well behaved gluing theory for buildings of this type. 


\section{Appendix. Counting boundary zeros}

In this appendix we define a $\frac{1}{2} \mathbb{Z}$-valued count of zeroes for sections of a complex line bundle with totally real boundary condition. Let $(E, J) \rightarrow S$ be a topological complex line bundle over a compact, connected and oriented surface with boundary. Partition the boundary into two open and closed subsets $\partial S=\partial_{0} S \sqcup \partial_{1} S$, either of which may be empty. Now choose a totally real subbundle $\left.\ell \subset E\right|_{\partial_{0} S} \rightarrow \partial_{0} S$, and consider the space of all continuous sections $\sigma: S \rightarrow E$ such that $\sigma\left(\partial_{0} S\right) \subset \ell$ and $\sigma \neq 0$ on $\partial_{1} S$. We will call such sections admissible. Suppose $\sigma$ is an admissible section with a discrete zero set $\sigma^{-1}(0) \subset S$. If $z_{0} \in \sigma^{-1}(0) \cap$ int $S$, then it is standard to define the order of the zero ord $\left(\sigma ; z_{0}\right)$ as the winding number of $\sigma$ over a small loop around $z_{0}$, computed in any local trivialization. The boundary condition makes it possible to extend this definition to isolated zeros on $\partial_{0} S$ as well: for $z_{0} \in \sigma^{-1}(0) \cap \partial_{0} S$, choose coordinates identifying a neighborhood $\mathcal{U}$ of $z_{0}$ with $\mathbb{D}^{+}=\{z \in \mathbb{C}|| z \mid \leq 1$ and $\operatorname{Im} z \geq 0\}$, such that $z_{0}=0$ and $\mathcal{U} \cap \partial S=\mathbb{D}^{+} \cap \mathbb{R}$. Choose also a local trivialization over $U$ that identifies $\ell$ with $\left(\mathbb{D}^{+} \cap \mathbb{R}\right) \times \mathbb{R} \subset \mathbb{D}^{+} \times \mathbb{C}$. Then $\sigma$ is represented on this neighborhood by a continuous function $f: \mathbb{D}^{+} \rightarrow \mathbb{C}$, satisfying the boundary condition $f\left(\mathbb{D}^{+} \cap \mathbb{R}\right) \subset \mathbb{R}$. We can therefore extend $f$ to a continuous function $f^{D}: \mathbb{D} \rightarrow \mathbb{C}$ on the full disk, satisfying $f^{D}(\bar{z})=\overline{f^{D}(z)}$. The order ord $\left(\sigma ; z_{0}\right)$ is then the order of the isolated zero of $f^{D}$ at 0 , i.e. the winding number of $f^{D}$ for a small circle about 0 . This definition does not depend on the choices.

For an admissible section $\sigma$ with discrete zero set $\sigma^{-1}(0)$, we now define the algebraic count of zeros by

$$
Z(\sigma)=\sum_{z \in \sigma^{-1}(0) \cap \operatorname{int} S} \operatorname{ord}(\sigma ; z)+\frac{1}{2} \sum_{z \in \sigma^{-1}(0) \cap \partial_{0} S} \operatorname{ord}(\sigma ; z) .
$$

Proposition A.1. Suppose $\sigma_{0}$ and $\sigma_{1}$ are admissible sections with isolated zeros, and are homotopic through a family of admissible sections. Then $Z\left(\sigma_{0}\right)=Z\left(\sigma_{1}\right)$.

Proof. This is clear if $\partial_{0} S=\emptyset$ : then $Z(\sigma)$ is the Euler number of $E$ if $\partial_{1} S=\emptyset$, or more generally the homotopy invariant winding number about $\partial_{1} S$ with respect to any global trivialization.

We reduce the general case to this by using a doubling argument: define the conjugate surface $S^{C}:=S$ with the opposite orientation, and the conjugate bundle $\left(E^{C}, J^{C}\right):=(E,-J) \rightarrow S^{C}$. Then we can glue $S$ to $S^{C}$ along $\partial_{0} S$ to define the doubled surface $S^{D}$, and similarly form a bundle $\left(E^{D}, J^{D}\right) \rightarrow S^{D}$ by gluing $(E, J)$ to $\left(E^{C}, J^{C}\right)$ via the unique complex bundle isomorphism $\left.\left.E\right|_{\partial_{0} S} \rightarrow E^{C}\right|_{\partial_{0} S}$ that restricts to the identity on $\ell$. Now $\partial S^{D}=\partial_{1} S^{D}=\partial_{1} S \cup \partial_{1} S^{C}$. Any admissible section $\sigma$ of $E$ defines an admissible section $\sigma^{D}$ of $E^{D}$, and the same statement 
applies to homotopies, thus it suffices to prove the following formula relating $Z(\sigma)$ to $Z\left(\sigma^{D}\right)$ :

$$
\begin{aligned}
Z\left(\sigma^{D}\right) & =\sum_{z \in\left(\sigma^{D}\right)^{-1}(0)} \operatorname{ord}\left(\sigma^{D} ; z\right) \\
& =2 \sum_{z \in \sigma^{-1}(0) \cap \operatorname{int} S} \operatorname{ord}(\sigma ; z)+\sum_{z \in \sigma^{-1}(0) \cap \partial_{0} S} \operatorname{ord}(\sigma ; z) \\
& =2 Z(\sigma) .
\end{aligned}
$$

This follows from two important facts which are easy to check: first, if $z$ is a zero of $\sigma$ in int $S$, its order is the same as that of the corresponding zero in $S^{C}$. Secondly, if $z$ is a boundary zero of $\sigma$, then its order equals its order as an interior zero of $\sigma^{D}$.

The doubling formula $Z\left(\sigma^{D}\right)=2 Z(\sigma)$ which emerged from this proof is a useful fact in itself; we shall apply it now to express $Z(\sigma)$ in terms of the relative first Chern number of $E$ and the boundary Maslov index of the pair $\left(\left.E\right|_{\partial_{0} S}, \ell\right)$.

Proposition A.2. For any choice of trivialization $\Phi$ along $\partial S$,

$$
Z(\sigma)=c_{1}^{\Phi}(E)+\frac{1}{2} \mu^{\Phi}(E, \ell)+\operatorname{wind}_{\partial_{1} S}^{\Phi}(\sigma) .
$$

Proof. Label the right hand side $\hat{Z}(\sigma)$ and observe that it does not depend on $\Phi$ and depends on $\sigma$ only up to homotopy through admissible sections. Moreover, it is clear that $Z(\sigma)=\hat{Z}(\sigma)$ if $\partial_{0} S=\emptyset$, so it will suffice to prove the doubling formula $\hat{Z}\left(\sigma^{D}\right)=2 \hat{Z}(\sigma)$. Since the orientations of both $E^{C}$ and $S^{C}$ are reversed, we have $\operatorname{wind}_{\partial_{1} S^{D}}\left(\sigma^{D}\right)=2 \operatorname{wind}_{\partial_{1} S}^{\Phi}(\sigma)$ for the natural trivialization $\Phi^{D}$ induced by $\Phi$. We claim also that

$$
c_{1}^{\Phi^{D}}\left(E^{D}\right)=2 c_{1}^{\Phi}(E)+\mu^{\Phi}(E, \ell),
$$

which will prove the result. This can be reduced to the standard additivity of the Maslov index under gluing. Construct a new surface $\bar{S} \supset S$ by gluing a disk to each component of $\partial_{1} S$, and glue in trivial bundles along $\Phi$ over these disks to produce a new bundle $(\bar{E}, \bar{J}) \rightarrow \bar{S}$, such that $\left.\bar{E}\right|_{S}=E$ and $c_{1}^{\Phi}(\bar{E})=c_{1}^{\Phi}(E)$. Now $\partial \bar{S}=\partial_{0} S$, and $2 c_{1}^{\Phi}(E)+\mu^{\Phi}(E, \ell)$ is by definition the absolute Maslov index $\mu(\bar{E}, \ell)$. (Alternatively, one can define the latter as $\mu^{\Phi}(\bar{E}, \ell)$ where $\Phi$ is any trivialization along $\partial \bar{S}$ that extends globally over $\bar{S}$.) Now the gluing property for $\mu(\bar{E}, \ell)$ gives

$$
2 \mu(\bar{E}, \ell)=\mu\left(\bar{E}^{D}\right)=2 c_{1}\left(\bar{E}^{D}\right)
$$

since $\bar{S}^{D}$ is closed. But the latter is also equal to $2 c_{1}^{\Phi^{D}}\left(E^{D}\right)$, proving the claim. 


\section{References}

[Bar00] J.-F. Barraud, Courbes pseudo-holomorphes équisingulières en dimension 4. Bull. Soc. Math. France 128 (2000), no. 2, 179-206. Zbl 0976.53037 MR 1772440351

[Bou02] F. Bourgeois, A Morse-Bott approach to contact homology. Ph.D. Thesis, Stanford University, 2002. 369

[BEH+03] F. Bourgeois, Y. Eliashberg, H. Hofer, K. Wysocki, and E. Zehnder, Compactness results in symplectic field theory. Geom. Topol. 7 (2003), 799-888. Zbl 1131.53312 MR 2026549 349, 350, 403

[BM04] F. Bourgeois and K. Mohnke, Coherent orientations in symplectic field theory. Math. Z. 248 (2004), no. 1, 123-146. Zbl 1060.53080 MR 2092725402

[Dra04] D. L. Dragnev, Fredholm theory and transversality for noncompact pseudoholomorphic maps in symplectizations. Comm. Pure Appl. Math. 57 (2004), no. 6, 726-763. Zbl 1063.53086 MR 2038115 369, 377

[EGH00] Y. Eliashberg, A. Givental, and H. Hofer, Introduction to symplectic field theory. Geom. Funct. Anal., Special Volume, 560-673. Zbl 0989.81114 MR 1826267396

[EKP06] Y. Eliashberg, S. S. Kim, and L. Polterovich, Geometry of contact transformations and domains: orderability versus squeezing. Geom. Topol. 10 (2006), 1635-1747 (electronic). Zbl 1134.53044 MR 2284048349

[Fra05] S. Francisco, Symplectic isotopy for cuspidal curves. Ph.D. Thesis, MIT, 2005. 351

[Gro85] M. Gromov, Pseudoholomorphic curves in symplectic manifolds. Invent. Math. 82 (1985), no. 2, 307-347. Zbl 0592.53025 MR 0809718348

[Hof93] H. Hofer, Pseudoholomorphic curves in symplectizations with applications to the Weinstein conjecture in dimension three. Invent. Math. 114 (1993), no. 3, 515-563. Zbl 0797.58023 MR 1244912 351, 354

[HLS97] H. Hofer, V. Lizan, and J.-C. Sikorav, On genericity for holomorphic curves in fourdimensional almost-complex manifolds. J. Geom. Anal. 7 (1997), no. 1, 149-159. Zbl 0911.53014 MR 1630789 347, 348, 354, 357

[HWZ95a] H. Hofer, K. Wysocki, and E. Zehnder, Properties of pseudo-holomorphic curves in symplectisations. II. Embedding controls and algebraic invariants. Geom. Funct. Anal. 5 (1995), no. 2, 270-328. Zbl 0845.57027 MR 1334869 361, 362

[HWZ95b] H. Hofer, K. Wysocki, and E. Zehnder, A characterisation of the tight three-sphere. Duke Math. J. 81 (1995), no. 1, 159-226. Zbl 0861.57026 MR 1470344355

[HWZ96] H. Hofer, K. Wysocki, and E. Zehnder, Properties of pseudoholomorphic curves in symplectisations. I. Asymptotics. Ann. Inst. H. Poincaré Anal. Non Linéaire 13 (1996), no. 3, 337-379. Zbl 0861.58018 MR 1395676 351, 362

[HWZ99] H. Hofer, K. Wysocki, and E. Zehnder, Properties of pseudoholomorphic curves in symplectizations. III. Fredholm theory. In Topics in nonlinear analysis, Progr. Nonlinear Differential Equations Appl. 35, Birkhäuser, Basel 1999, 381-475. Zbl 0924.58003 MR 1725579 348, 357, 361

[HWZ03] H. Hofer, K. Wysocki, and E. Zehnder, Finite energy foliations of tight threespheres and Hamiltonian dynamics. Ann. of Math. (2) 157 (2003), no. 1, 125-255. Zbl 01933157 MR 1954266 349, 395 
[IS99] S. Ivashkovich and V. Shevchishin, Structure of the moduli space in a neighborhood of a cusp-curve and meromorphic hulls. Invent. Math. 136 (1999), no. 3, 571-602. Zbl 0930.32017 MR 1695206 347, 349, 354, 357, 378

[MS04] D. McDuff and D. Salamon, J-holomorphic curves and symplectic topology. Amer. Math. Soc. Colloq. Publ. 52, Amer. Math. Soc., Providence, RI, 2004. Zbl 1064.53051 MR 2045629 353, 355

[MW95] M. J. Micallef and B. White, The structure of branch points in minimal surfaces and in pseudoholomorphic curves. Ann. of Math. (2) 141 (1995), no. 1, 35-85. Zbl 0873.53038 MR 1314031 389

[RS95] J. Robbin and D. Salamon, The spectral flow and the Maslov index. Bull. London Math. Soc. 27 (1995), no. 1, 1-33. Zbl 0859.58025 MR 1331677370

[Sch95] M. Schwarz, Cohomology operations from $S^{1}$-cobordisms in Floer homology. Ph.D. Thesis, ETH Zürich, 1995. 361, 364

[She01] V. Shevchishin, Pseudoholomorphic curves and the symplectic isotopy problem. Habilitation Thesis, Bochum, 2001; preprint 2000. arXiv:math/0010262. 355

[Sie08] R. Siefring, Relative asymptotic behavior of pseudoholomorphic half-cylinders. Comm. Pure Appl. Math. 61 (2008), no. 12, 1631-1684. Zbl 1158.53068 MR 2456182 362, 387, 389

[Sie08] R. Siefring, Intersection theory of punctured pseudoholomorphic curves. Preprint 2009. arXiv:0907.0470v1 387, 388, 391

[SW] R. Siefring and C. Wendl, Pseudoholomorphic curves, intersections and Morse-Bott asymptotics. In preparation. 387, 388, 391

[Sik03] J.-C. Sikorav, The gluing construction for normally generic $J$-holomorphic curves. In Symplectic and contact topology: interactions and perspectives, Fields Inst. Commun. 35, Amer. Math. Soc., Providence, RI, 2003, 175-199. Zbl 1109.32301 MR 1969276355

[Tro92] A. J. Tromba, Teichmüller theory in Riemannian geometry. Lectures Math. ETH Zurich, Birkhäuser, Basel 1992. Zbl 0785.53001 MR 1164870366

[Wen05] C. Wendl, Finite energy foliations and surgery on transverse links. Ph.D. Thesis, New York University, 2005. 348, 354, 361

[Wen08] C. Wendl, Finite energy foliations on overtwisted contact manifolds. Geom. Topol. 12 (2008), 531-616. Zbl 1141.53082 MR 2390353 349, 355

[Wena] C. Wendl, Compactness for embedded pseudoholomorphic curves in 3-manifolds. J. Eur. Math. Soc., to appear; preprint 2007. arXiv:math/0703509. 351, 356, 357, 391

[Wenb] C. Wendl, Punctured holomorphic curves with boundary in 3-manifolds: Fredholm theory and embededdness. In preparation. 351, 355, 361, 371, 402

Received April 7, 2008

Chris Wendl, Institut für Mathematik, Humboldt-Universität zu Berlin, 10099 Berlin, Germany

E-mail: wendl@math.hu-berlin.de 